BULLETIN (New Series) OF THE

AMERICAN MATHEMATICAL SOCIETY

Volume 48, Number 3, July 2011, Pages 325-407

S 0273-0979(2011)01334-9

Article electronically published on March 25, 2011

\title{
THE CLASSICAL THEORY OF MINIMAL SURFACES
}

\author{
WILLIAM H. MEEKS III AND JOAQUÍN PÉREZ
}

\begin{abstract}
We present here a survey of recent spectacular successes in classical minimal surface theory. We highlight this article with the theorem that the plane, the helicoid, the catenoid and the one-parameter family $\left\{\mathcal{R}_{t}\right\}_{t \in(0,1)}$ of Riemann minimal examples are the only complete, properly embedded, minimal planar domains in $\mathbb{R}^{3}$; the proof of this result depends primarily on work of Colding and Minicozzi, Collin, López and Ros, Meeks, Pérez and Ros, and Meeks and Rosenberg. Rather than culminating and ending the theory with this classification result, significant advances continue to be made as we enter a new golden age for classical minimal surface theory. Through our telling of the story of the classification of minimal planar domains, we hope to pass on to the general mathematical public a glimpse of the intrinsic beauty of classical minimal surface theory and our own perspective of what is happening at this historical moment in a very classical subject.
\end{abstract}

\section{Contents}

1. Introduction

2. Basic results in classical minimal surface theory

3. Minimal surfaces with finite topology and more than one end 355

4. Sequences of embedded minimal surfaces without uniform local area bounds

5. The structure of minimal laminations of $\mathbb{R}^{3}$

6. The ordering theorem for the space of ends

7. Conformal structure of minimal surfaces

9. Minimal laminations revisited and the embedded Calabi-Yau problem

10. Embedded minimal surfaces of finite genus

Received by the editors September 4, 2006 and, in revised form, October 15, 2007, November 30, 2009, and February 2, 2011.

2010 Mathematics Subject Classification. Primary 53A10; Secondary 49Q05, 53C42.

Key words and phrases. Minimal surface, minimal lamination, locally simply connected, finite total curvature, conformal structure, harmonic function, recurrence, transience, parabolic Riemann surface, harmonic measure, universal superharmonic function, Jacobi function, stability, index of stability, Shiffman function, Korteweg-de Vries equation, KdV hierarchy, algebrogeometric potential, curvature estimates, maximum principle at infinity, limit tangent plane at infinity, parking garage, minimal planar domain.

This material is based upon work for the NSF under Awards No. DMS - 0405836, DMS 0703213, DMS - 1004003. Any opinions, findings, and conclusions or recommendations expressed in this publication are those of the authors and do not necessarily reflect the views of the NSF.

Research partially supported by a Spanish MEC-FEDER Grant no. MTM2007-61775 and a Regional J. Andalucía Grant no. P06-FQM-01642.

(C)2011 American Mathematical Society 325

Reverts to public domain 28 years from publication 
11. Outstanding problems and conjectures 390

About the authors $\quad 398$

Acknowledgments $\quad 399$

References $\quad 399$

\section{INTRODUCTION}

The theory of minimal surfaces in three-dimensional Euclidean space has its roots in the calculus of variations developed by Euler and Lagrange in the 18th century and in later investigations by Enneper, Scherk, Schwarz, Riemann and Weierstrass in the 19th century. Over the years, many great mathematicians have contributed to this theory. Besides the above-mentioned names that belong to the nineteenth century, we find fundamental contributions by Bernstein, Courant, Douglas, Morrey, Morse, Radó and Shiffman in the first half of the last century. Paraphrasing Osserman, most of the activity in minimal surface theory in those days was focused almost exclusively on either Plateau's problem or PDE questions, and the only global result was the negative one of Bernstein's theorem 1

Much of the modern global theory of complete minimal surfaces in three-dimensional Euclidean space has been influenced by the pioneering work of Osserman during the 1960s. Many of the global questions and conjectures that arose in this classical subject have only recently been addressed. These questions concern analytic and conformal properties, the geometry and asymptotic behavior, and the topology and classification of the images of certain injective minimal immersions $f: M \rightarrow \mathbb{R}^{3}$ which are complete in the induced Riemannian metric; we call the image of such a complete, injective, minimal immersion a complete, embedded minimal surface in $\mathbb{R}^{3}$.

The following classification results solve two of these long standing conjectures 2

Theorem 1.1. A complete, embedded, simply connected minimal surface in $\mathbb{R}^{3}$ is a plane or a helicoid.

Theorem 1.2. Up to scaling and rigid motion, any connected, properly embedded, minimal planar domain in $\mathbb{R}^{3}$ is a plane, a helicoid, a catenoid or one of the Riemann minimal examples 3 In particular, for every such surface there exists a foliation of $\mathbb{R}^{3}$ by parallel planes, each of which intersects the surface transversely in a connected curve which is a circle or a line.

The proofs of the above theorems depend on a series of new results and theory that have been developed over the past decade. The purpose of this article is twofold. The first is to explain these new global results in a manner accessible to the general mathematical public, and the second is to explain how these results transcend their application to the proofs of Theorems 1.1 and 1.2 and enhance

\footnotetext{
${ }^{1}$ This celebrated result by Bernstein 6] asserts that the only minimal graphs over the entire plane are graphs given by affine functions, which means that the graphs are planes.

${ }^{2}$ Several authors pointed out to us that Osserman seems to be the first to ask the question about whether the plane and the helicoid were the only embedded, simply connected, complete minimal surfaces. He described this question as potentially the most beautiful extension and explanation of Bernstein's Theorem.

${ }^{3}$ The Riemann minimal examples referred to here were discovered by Riemann around 1860. See Section 2.5 for a further discussion of these surfaces and images of them.
} 
dramatically our current understanding of the theory, giving rise to new theorems and conjectures, some of which were considered to be almost unapproachable dreams just 15 years ago. The interested reader can also find more detailed history and further results in the following surveys, reports and popular science articles [3, 12, 30, 36, 45, 46, 71, 72, 74, 76, 81, 107, 113, 117, 158, 173, 182. We refer the reader to the excellent graduate texts on minimal surfaces by Dierkes et al 47, 48, Lawson 99] and Osserman [163, and especially see Nitsche's book 160] for a fascinating account of the history and foundations of the subject.

Before proceeding, we make a few general comments on the proof of Theorem 1.1 which we feel can suggest to the reader a visual idea of what is going on. The most natural motivation for understanding this theorem, Theorem 1.2 and other results presented in this survey is to try to answer the following question: What are the possible shapes of surfaces which satisfy a variational principle and have a given topology? For instance, if the variational equation expresses the critical points of the area functional, and if the requested topology is the simplest one of a disk, then Theorem 1.1 says that the possible shapes for complete examples are the trivial one given by a plane and (after a rotation) an infinite double spiral staircase, which is a visual description of a vertical helicoid. A more precise description of the double spiral staircase nature of a vertical helicoid is that this surface is the union of two infinite-sheeted multigraphs (see Definition 4.1 for the notion of a multigraph) that are glued along a vertical axis. Crucial in the proof of Theorem 1.1 are local results of Colding and Minicozzi which describe the structure of compact, embedded minimal disks, as essentially being modeled by one of the above two examples; i.e., either they are graphs or pairs of finitely sheeted multigraphs glued along an "axis". A last key ingredient in the proof of Theorem 1.1 is a result on the global aspects of limits of these shapes, which is given in the Lamination Theorem for Disks by Colding and Minicozzi; see Theorem 4.2 below.

For the reader's convenience, we next include a guide of how the sections depend on each other; see below for a more detailed explanation of their contents.

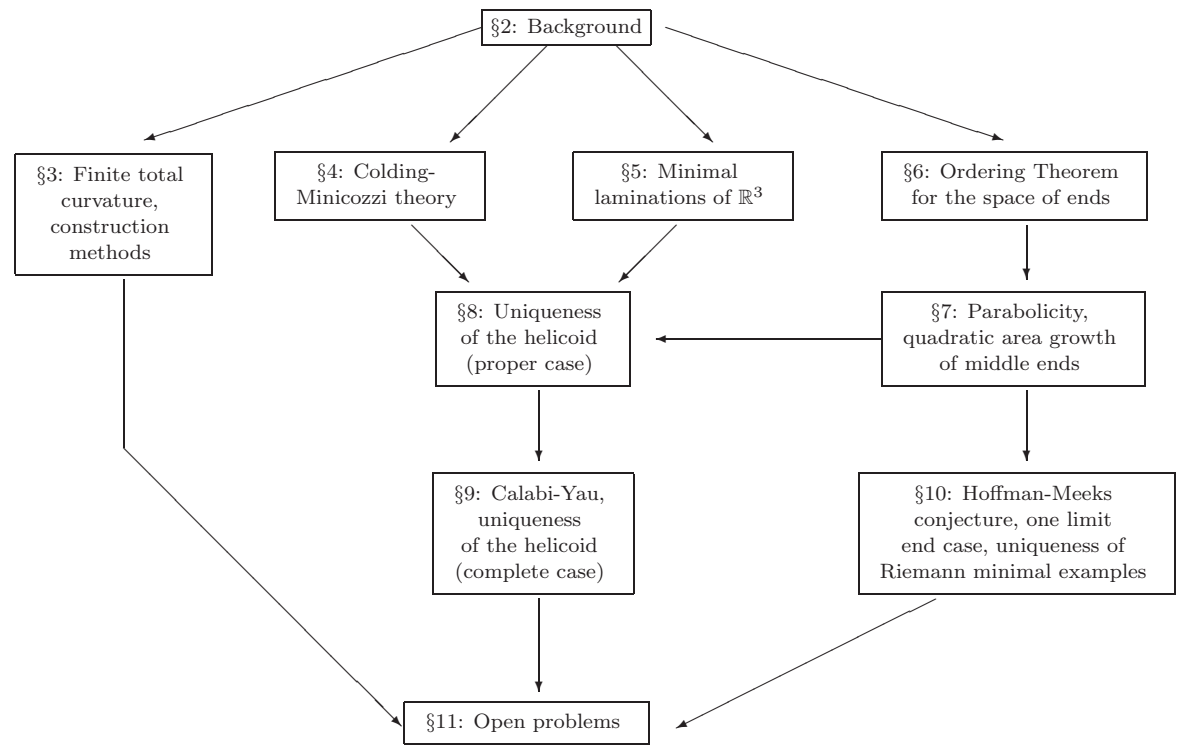


Our survey is organized as follows. We present the main definitions and background material in the introductory Section 2. In that section we also briefly describe geometrically, as well as analytically, many of the important classical examples of properly embedded minimal surfaces in $\mathbb{R}^{3}$. As in many other areas in mathematics, understanding key examples in this subject is crucial in obtaining a feeling for the subject, making important theoretical advances and asking the right questions. We believe that before going further, the unacquainted reader to this subject will benefit by taking a few minutes to view and identify the computer graphics images of these surfaces, which appear near the end of Section 2.5. and to read the brief historical and descriptive comments related to the individual images.

In Section 3 we describe the best understood family of complete embedded minimal surfaces: those with finite topology and more than one end. Recall that a compact, orientable surface is homeomorphic to a connected sum of tori, and the number of these tori is called the genus of the surface. An (orientable) surface $M$ of finite topology is one which is homeomorphic to a compact surface of genus $g \in \mathbb{N} \cup\{0\}$ with finitely many points removed, called the ends of $M$. These punctures can be naturally identified with different ways to escape to infinity on $M$, and also can be identified with punctured disk neighborhoods of these points. These punctured disk neighborhoods are clearly annuli; hence they are called annular ends. The crucial result for minimal surfaces with finite topology and more than one end is Collin's Theorem, valid under the additional assumption of properness (a surface in $\mathbb{R}^{3}$ is proper if each closed ball in $\mathbb{R}^{3}$ contains a compact portion of the surface with respect to its intrinsic topology). Note that properness implies completeness.

Theorem 1.3 (Collin 38). If $M \subset \mathbb{R}^{3}$ is a properly embedded minimal surface with more than one end, then each annular end of $M$ is asymptotic to the end of a plane or a catenoid. In particular, if $M$ has finite topology and more than one end, then $M$ has finite total Gaussian curvature 4

Collin's Theorem reduces the analysis of properly embedded minimal surfaces of finite topology and more than one end in $\mathbb{R}^{3}$ to complex function theory on compact Riemann surfaces. This reduction then leads to classification results and to interesting topological obstructions, which we include in Section 3 as well. At the end of Section 3. we discuss several different methods for constructing properly embedded minimal surfaces of finite topology and for describing their moduli spaces.

In Section 4. we present an overview of some results concerning the geometry, compactness and regularity of limits of what are called locally simply connected sequences of minimal surfaces. These results are central in the proofs of Theorems 1.1 and 1.2 and are taken from a series of papers by Colding and Minicozzi 27, 31, 32, 33, 34, 37.

In Sections 4 and 5 we define and develop the notion of minimal lamination, which is a natural limit object for a locally simply connected sequence of embedded minimal surfaces. The reader not familiar with the subject of minimal laminations should think about the closure of an embedded, non-compact geodesic $\gamma$ on a complete Riemannian surface, a topic which has been widely covered in the literature (see, e.g., Bonahon 9]). The closure of such a geodesic $\gamma$ is a geodesic lamination $\mathcal{L}$ of the surface. When $\gamma$ has no accumulation points, then it is proper and it is

\footnotetext{
${ }^{4}$ See equation (3) for the definition of total curvature.
} 
the unique leaf of $\mathcal{L}$. Otherwise, there pass complete, embedded, pairwise disjoint geodesics through the accumulation points, and these geodesics together with $\gamma$ form the leaves of the geodesic lamination $\mathcal{L}$. A similar result is true for a complete, embedded minimal surface of locally bounded curvature (i.e., whose Gaussian curvature is bounded in compact extrinsic balls) in a Riemannian three-manifold [136]. We include in Section 5 a discussion of the Structure Theorem for Minimal Laminations of $\mathbb{R}^{3}$ (Meeks-Rosenberg [136] and Meeks-Pérez-Ros [126]).

In Section [6] we explain the Ordering Theorem of Frohman and Meeks 61 for the linear ordering of the ends of any properly embedded minimal surface with more than one end; this is a fundamental result for our purposes of classifying embedded, minimal planar domains in Theorem 1.2

Section 7 is devoted to conformal questions on minimal surfaces. Roughly speaking, this means studying the conformal type of a given minimal surface (rather than its Riemannian geometry) considered as a Riemann surface, i.e., an orientable surface in which one can find an atlas by charts with a holomorphic change of coordinates. To do this, we first define the notion of universal superharmonic function for domains in $\mathbb{R}^{3}$ and give examples. Next we explain how to use these functions to understand the conformal structure of properly immersed minimal surfaces in $\mathbb{R}^{3}$ which fail to have finite topology. We then follow the work of Collin, Kusner, Meeks and Rosenberg in 39 to analyze the asymptotic geometry and conformal structure of properly embedded minimal surfaces of infinite topology.

In Section 8, we apply the results in the previous sections to explain the main steps in the proof of Theorem 1.1 after replacing completeness by the stronger hypothesis of properness. This theorem together with Theorem 1.3 above, Theorem 1.5 below and with results by Bernstein and Breiner [4] or by Meeks and Pérez [115] lead to a complete understanding of the asymptotic geometry of any annular end of a complete, embedded minimal surface with finite topology in $\mathbb{R}^{3}$; namely, the annular end must be asymptotic to an end of a plane, catenoid or helicoid. For a discussion of the proof of Theorem 1.4 in the case of positive genus and a more general classification result of complete embedded minimal annular ends with compact boundary and infinite total curvature in $\mathbb{R}^{3}$, see the monograph [116].

Theorem 1.4. Every properly embedded, non-planar minimal surface in $\mathbb{R}^{3}$ with finite genus and one end has the conformal structure of a compact Riemann surface $\Sigma$ minus one point, can be analytically represented by meromorphic data on $\Sigma$ and is asymptotic to a helicoid. Furthermore, when the genus of $\Sigma$ is zero, the only possible examples are helicoids.

In Section 9. we complete our sketch of the proof of Theorem 1.1 by allowing the surface to be complete rather than proper. The problem of understanding the relation between the intrinsic notion of completeness and the extrinsic one of properness is known as the embedded Calabi-Yau problem in minimal surface theory; see [13, [21, 210], 211] and [152] for the original Calabi-Yau problem in the complete immersed setting. Along these lines, we also describe the powerful Minimal Lamination Closure Theorem (Meeks-Rosenberg [137, Theorem 9.2 below) and other related results. Theorem 9.2 is a refinement of the results and techniques used by Colding and Minicozzi [37] to prove the following deep result (see Section 9, where we deduce Theorem 1.5 from Theorem 9.2). 
Theorem 1.5 (Colding, Minicozzi [37]). A complete, embedded minimal surface of finite topology in $\mathbb{R}^{3}$ is properly embedded.

With Theorem 1.5 in hand, we note that the hypothesis of properness for $M$ in the statements of Theorem 1.4 and of Collin's Theorem 1.3 can be replaced by the weaker hypothesis of completeness for $M$. Hence, Theorem 1.1 follows from Theorems 1.4 and 1.5 .

In Section 10, we present some of the results of Meeks, Pérez and Ros 119, 120, 124, 126, 127. on the geometry of complete, embedded minimal surfaces of finite genus with possibly an infinite number of ends, including the proof of Theorem 1.2 stated above. We first explain Theorem 1.6 below on the existence of a bound on the number of ends of a complete, embedded minimal surface with finite total curvature solely in terms of the genus. So far, this is the best result towards the solution of the so-called Hoffman-Meeks conjecture:

A connected surface of finite topology, genus $g$ and $r$ ends, $r>2$, can be properly minimally embedded in $\mathbb{R}^{3}$ if and only if $r \leq g+2$.

Theorem 1.6 (Meeks, Pérez, Ros [119]). For every non-negative integer g, there exists an integer $e(g)$ such that if $M \subset \mathbb{R}^{3}$ is a complete, embedded minimal surface of finite topology with genus $g$, then the number of ends of $M$ is at most $e(g)$.

The next goal of Section 10 is to focus on the proof of Theorem 1.2. In this setting of infinitely many ends, the set of ends has a topological structure which makes it a compact, total disconnected metric space with infinitely many points; see Definition 2.20 and the paragraph below it. This set has accumulation points, which produce the new notion of limit end. The first step in the proof of Theorem 1.2 is to notice that the number of limit ends of a properly embedded minimal surface in $\mathbb{R}^{3}$ is at most 2 ; this result appears as Theorem [7.9. Then one rules out the existence of a properly embedded minimal planar domain with just one limit end: this is the purpose of Theorem 10.3 At that point, we are ready to finish the proof of Theorem 1.2. This breaks into two parts, the first of which is a quasi-periodicity property coming from curvature estimates for any surface satisfying the hypotheses of Theorem 1.2 (see [126]), and the second one is based on the Shiffman function and its relation to integrable systems theory through the Korteweg-de Vries (KdV) equation; see Theorem 10.6 below.

The final section of this survey is devoted to a discussion of some of the main outstanding conjectures in the subject. Many of these problems are motivated by the recent advances in classical minimal surface theory reported on in previous sections, and many of them appear in print for the first time here. Research mathematicians, not necessarily schooled in differential geometry, are likely to find some of these problems accessible to attack with methods familiar to them. We invite anyone with an inquisitive mind and strong geometrical intuition to join in the game of trying to solve these intriguing open problems.

For an inclusive discussion of recent advances in the classical theory of minimal surfaces, we refer the reader to our monograph [116] on which the present manuscript is based. The central topics in [116] include the following:

(1) The topological classification of minimal surfaces in $\mathbb{R}^{3}$ by Frohman and Meeks [57.

(2) The uniqueness of Scherk's singly periodic minimal surfaces by Meeks and Wolf 143 . 
(3) The Calabi-Yau problem for minimal surfaces based on work by Nadirashvili 152 and Ferrer, Martín and Meeks [58].

(4) Colding-Minicozzi theory for minimal surfaces of finite genus 24.

(5) The asymptotic behavior of minimal annular ends by Meeks and Pérez [115.

(6) The local removable singularity theorem for minimal laminations and its application to the proofs of the quadratic decay of curvature theorem, the dynamics theorem for minimal surfaces and the local picture theorem on the scale of topology by Meeks, Pérez and Ros [122.

\section{BASIC RESULTS IN CLASSICAL MINIMAL SURFACE THEORY}

We will devote this section to giving a fast tour through the foundations of the theory, enough to understand the results to be explained in future sections.

2.1. Eight equivalent definitions of minimality. One can define a minimal surface from different points of view. The equivalences between these starting points give insight into the richness of the classical theory of minimal surfaces and its connections with other branches of mathematics.

Definition 2.1. Let $X=\left(x_{1}, x_{2}, x_{3}\right): M \rightarrow \mathbb{R}^{3}$ be an isometric immersion of a Riemannian surface into space. $X$ is said to be minimal if $x_{i}$ is a harmonic function on $M$ for each $i$. In other words, $\Delta x_{i}=0$, where $\Delta$ is the Riemannian Laplacian on $M$.

Very often, it is useful to identify a Riemannian surface $M$ with its image under an isometric embedding. Since harmonicity is a local concept, the notion of minimality can be applied to an immersed surface $M \subset \mathbb{R}^{3}$ (with the underlying Riemannian structure induced by the inclusion). Let $H$ be the mean curvature function of $X$, which at every point is the average normal curvature, and let $N: M \rightarrow \mathbb{S}^{2} \subset \mathbb{R}^{3}$ be its unit normal or Gauss map 5 The well-known vector-valued formula $\Delta X=2 H N$, valid for an isometric immersion $X: M \rightarrow \mathbb{R}^{3}$, leads us to the following equivalent definition of minimality.

Definition 2.2. A surface $M \subset \mathbb{R}^{3}$ is minimal if and only if its mean curvature vanishes identically.

After rotation, any (regular) surface $M \subset \mathbb{R}^{3}$ can be locally expressed as the graph of a function $u=u(x, y)$. In 1776, Meusnier [146] discovered that the condition on the mean curvature to vanish identically can be expressed as the following quasi-linear, second-order, elliptic partial differential equation, found in 1762 by Lagrang $€$ [97:

$$
\left(1+u_{x}^{2}\right) u_{y y}-2 u_{x} u_{y} u_{x y}+\left(1+u_{y}^{2}\right) u_{x x}=0,
$$

which admits a divergence form version:

$$
\operatorname{div}\left(\frac{\nabla u}{\sqrt{1+|\nabla u|^{2}}}\right)=0
$$

Definition 2.3. A surface $M \subset \mathbb{R}^{3}$ is minimal if and only if it can be locally expressed as the graph of a solution of the equation (1).

\footnotetext{
${ }^{5}$ Throughout the paper, all surfaces will be assumed to be orientable unless otherwise stated.

${ }^{6}$ In reality, Lagrange arrived at a slightly different formulation, and equation (1) was derived five years later by Borda 11 .
} 
Let $\Omega$ be an orientable subdomain with compact closure in a surface $M \subset \mathbb{R}^{3}$. If we perturb normally the inclusion map $X$ on $\Omega$ by a compactly supported smooth function $u \in C_{0}^{\infty}(\Omega)$, then $X+t u N$ is again an immersion whenever $|t|<\varepsilon$, with $\varepsilon$ sufficiently small. The mean curvature function $H$ of $M$ relates to the infinitesimal variation of the area functional $A(t)=\operatorname{Area}((X+t u N)(\Omega))$ for compactly supported normal variations by means of the first variation of area (see, for instance, [160]):

$$
A^{\prime}(0)=-2 \int_{\Omega} u H d A
$$

where $d A$ stands for the area element of $M$. This variational formula lets us state a fourth equivalent definition of minimality.

Definition 2.4. A surface $M \subset \mathbb{R}^{3}$ is minimal if and only if it is a critical point of the area functional for all compactly supported variations.

In fact, a consequence of the second variation of area (Section 2.8) is that any point in a minimal surface has a neighborhood with least-area relative to its boundary. This property justifies the word "minimal" for these surfaces. It should be noted that the global minimization of area on every compact subdomain is a strong condition for a complete minimal surface to satisfy; in fact, it forces the surface to be a plane (Theorem 2.25).

Definition 2.5. A surface $M \subset \mathbb{R}^{3}$ is minimal if and only if every point $p \in M$ has a neighborhood with least-area relative to its boundary.

Definitions 2.4 and 2.5 establish minimal surfaces as the two-dimensional analog to geodesics in Riemannian geometry, and connect the theory of minimal surfaces with one of the most important classical branches of mathematics: the calculus of variations. Besides the area functional $A$, another well-known functional in the calculus of variations is the Dirichlet energy,

$$
E=\int_{\Omega}|\nabla X|^{2} d A,
$$

where again $X: M \rightarrow \mathbb{R}^{3}$ is an isometric immersion and $\Omega \subset M$ is a subdomain with compact closure. These functionals are related by the inequality $E \geq 2 A$, with equality if and only if the immersion $X: M \rightarrow \mathbb{R}^{3}$ is conformal. The classical formula $K-e^{2 u} \bar{K}=\Delta u$ that relates the Gaussian curvature functions $K, \bar{K}$ for two conformally related metrics $g, \bar{g}=e^{2 u} g$ on a two-dimensional manifold ( $\Delta$ stands for the Laplace operator with respect to $g$ ), together with the existence of solutions of the Laplace equation $\Delta u=K$ for any open subdomain with compact closure $\Omega$ in a Riemannian manifold $M$ such that the volume of $M-\Omega$ is positive, guarantees the existence of local isothermal or conformal coordinates for any two-dimensional Riemannian manifold, modeled on domains of $\mathbb{C}$. The relation between area and energy, together with the existence of isothermal coordinates, allows us to give two further characterizations of minimality.

Definition 2.6. A conformal immersion $X: M \rightarrow \mathbb{R}^{3}$ is minimal if and only if it is a critical point of the Dirichlet energy for all compactly supported variations, or equivalently if any point $p \in M$ has a neighborhood with least energy relative to its boundary.

From a physical point of view, the mean curvature function of a homogeneous membrane separating two media is equal, up to a non-zero multiplicative constant, 
to the difference between the pressures at the two sides of the surface (LaplaceYoung equation). When this pressure difference is zero, then the membrane has zero mean curvature. Therefore, soap films (i.e., not bubbles) in space are physical realizations of the ideal concept of a minimal surface.

Definition 2.7. A surface $M \subset \mathbb{R}^{3}$ is minimal if and only if every point $p \in M$ has a neighborhood $D_{p}$ which is equal to the unique idealized soap film with boundary $\partial D_{p}$.

Consider again the Gauss map $N: M \rightarrow \mathbb{S}^{2}$ of $M$. Then, the tangent space $T_{p} M$ of $M$ at $p \in M$ can be identified as subspace of $\mathbb{R}^{3}$ under parallel translation with the tangent space $T_{N(p)} \mathbb{S}^{2}$ to the unit sphere at $N(p)$. Hence, one can view the differential $A_{p}=-d N_{p}$ as an endomorphism of $T_{p} M$, called the shape operator. $A_{p}$ is a symmetric linear transformation, whose orthogonal eigenvectors are called the principal directions of $M$ at $p$, and the corresponding eigenvalues are the principal curvatures of $M$ at $p$. Since the mean curvature function $H$ of $M$ equals the arithmetic mean of such principal curvatures, minimality reduces to the following expression:

$$
A_{p}=-d N_{p}=\left(\begin{array}{cc}
a & b \\
b & -a
\end{array}\right)
$$

in an orthonormal tangent basis. After identification of $N$ with its stereographic projection, the Cauchy-Riemann equations give the next and last characterization of minimality.

Definition 2.8. A surface $M \subset \mathbb{R}^{3}$ is minimal if and only if its stereographically projected Gauss map $g: M \rightarrow \mathbb{C} \cup\{\infty\}$ is meromorphic with respect to the underlying Riemann surface structure.

This concludes our discussion of the equivalent definitions of minimality. The connection between minimal surface theory and complex analysis made possible the advances in the so-called first golden age of classical minimal surface theory (approximately 1855-1890). In this period, many great mathematicians took part, such as Beltrami, Bonnet, Catalan, Darboux, Enneper, Lie, Riemann, Schoenflies, Schwarz, Serret, Weierstrass, Weingarten, etc. (these historical references and many others can be found in the excellent book by Nitsche [160]). A second golden age of classical minimal surface theory occurred in the decade 1930-1940, with pioneering works by Courant, Douglas, Morrey, Morse, Radó, Shiffman and others. Among the greatest achievements of this period, we mention that Douglas won the first Fields medal (jointly with Ahlfors) for his complete solution to the classical Plateau problem 7 for disks.

Many geometers believe that since the early 1980s, we are living in a third golden age of classical minimal surface theory. A vast number of new embedded examples has been found in this period, very often with the aid of modern computers, which allow us to visualize beautiful models such as those appearing in the figures of this text. In these years, geometric measure theory, conformal geometry, functional analysis, integrable systems and other branches of mathematics have joined the classical methods, contributing fruitful new techniques and results, some of which will be treated in this article. At the same time, the theory of minimal surfaces

\footnotetext{
${ }^{7}$ In its simplest formulation, this problem asks whether any smooth Jordan curve in $\mathbb{R}^{3}$ bounds a disk of least area, a problem proposed by the Belgian physicist Plateau in 1870 .
} 
has diversified and expanded its frontiers. Minimal submanifolds in more general ambient geometries have been studied, and subsequent applications of minimal submanifolds have helped lead to solutions of some fundamental problems in other branches of mathematics, including the Positive Mass Conjecture (Schoen, Yau) and the Penrose Conjecture (Bray) in mathematical physics, and the Smith Conjecture (Meeks, Yau), the Poincaré Conjecture (Colding, Minicozzi, Perelman) and the Thurston Geometrization Conjecture in three-manifold theory.

Returning to our background discussion, we note that Definition 2.1 and the maximum principle for harmonic functions imply that no compact minimal surfaces in $\mathbb{R}^{3}$ exist. Although the study of compact minimal surfaces with boundary has been extensively developed and dates back to the well-known Plateau problem (see Footnote 7), in this survey we will focus on the study of complete minimal surfaces (possibly with boundary), in the sense that all geodesics can be indefinitely extended up to the boundary of the surface. Note that, with respect to the natural Riemannian distance function between points on a surface, the property of being "geodesically complete" is equivalent to the surface being a complete metric space. A stronger global hypothesis, whose relationship with completeness is an active field of research in minimal surface theory, is presented in the following definition.

Definition 2.9. A map $f: X \rightarrow Y$ between topological spaces is proper if $f^{-1}(C)$ is compact in $X$ for any compact set $C \subset Y$. A minimal surface $M \subset \mathbb{R}^{3}$ is proper when the inclusion map is proper.

The Gaussian curvature function $K$ of a surface $M \subset \mathbb{R}^{3}$ is the product of its principal curvatures. If $M$ is minimal, then its principal curvatures are oppositely signed and thus, $K$ is non-positive. Another interpretation of $K$ is the determinant of the shape operator $A$; thus $|K|$ is the absolute value of the Jacobian for the Gauss map $N$. Therefore, after integrating $K$ on $M$ (note that this integral may be $-\infty$ or a non-positive number), we obtain the same quantity as when computing the negative of the spherical area of $M$ through its Gauss map, counting multiplicities. This quantity is called the total curvature of the minimal surface:

$$
C(M)=\int_{M} K d A=-\operatorname{Area}\left(N: M \rightarrow \mathbb{S}^{2}\right) .
$$

2.2. Weierstrass representation. Recall that the Gauss map of a minimal surface $M$ can be viewed as a meromorphic function $g: M \rightarrow \mathbb{C} \cup\{\infty\}$ on the underlying Riemann surface. Furthermore, the harmonicity of the third coordinate function $x_{3}$ of $M$ lets us define (at least locally) its harmonic conjugate function $x_{3}^{*}$; hence, the so-called height differentia $8 d h=d x_{3}+i d x_{3}^{*}$ is a holomorphic differential on $M$. The pair $(g, d h)$ is usually referred to as the Weierstrass datd 9 of the minimal surface, and the minimal immersion $X: M \rightarrow \mathbb{R}^{3}$ can be expressed up to translation by $X\left(p_{0}\right), p_{0} \in M$, solely in terms of this data as

$$
X(p)=\Re \int_{p_{0}}^{p}\left(\frac{1}{2}\left(\frac{1}{g}-g\right), \frac{i}{2}\left(\frac{1}{g}+g\right), 1\right) d h,
$$

where $\Re$ stands for real part [99, 163]. The pair $(g, d h)$ satisfies certain compatibility conditions, stated in assertions i), ii) of Theorem 2.10 below. The key point is that

\footnotetext{
${ }^{8}$ Note that the height differential might not be exact since $x_{3}^{*}$ need not be globally well defined on $M$. Nevertheless, the notation $d h$ is commonly accepted, and we will also make use of it here.

${ }^{9}$ This representation was derived locally by Weierstrass in 1866 .
} 
this procedure has the following converse, which gives a cookbook-type recipe for analytically defining a minimal surface.

Theorem 2.10 (Osserman [161]). Let $M$ be a Riemann surface, $g: M \rightarrow \mathbb{C} \cup\{\infty\}$ a meromorphic function and dh a holomorphic one-form on $M$. Assume that:

i) The zeros of dh coincide with the poles and zeros of $g$, with the same order.

ii) For any closed curve $\gamma \subset M$,

$$
\overline{\int_{\gamma} g d h}=\int_{\gamma} \frac{d h}{g}, \quad \Re \int_{\gamma} d h=0,
$$

where $\bar{z}$ denotes the complex conjugate of $z \in \mathbb{C}$. Then, the map $X: M \rightarrow \mathbb{R}^{3}$ given by (44) is a conformal minimal immersion with Weierstrass data $(g, d h)$.

Condition $i$ ) in Theorem 2.10 expresses the non-degeneracy of the induced metric by $X$ on $M$, so by weakening it to the condition that the zeros and poles of $g$ coincide with the zeros of $d h$ with at most the same order, we allow the conformal $X$ to be a branched minimal surface. Condition ii) deals with the independence of (4) on the integration path, and it is usually called the period problem 10 By the Divergence Theorem, it suffices to consider the period problem on homology classes in $M$.

All local geometric invariants of a minimal surface $M$ can be expressed in terms of its Weierstrass data. For instance, the first and second fundamental forms are respectively (see [74, 163):

$$
d s^{2}=\left(\frac{1}{2}\left(|g|+|g|^{-1}\right)|d h|\right)^{2}, \quad I I(v, v)=\Re\left(\frac{d g}{g}(v) \cdot d h(v)\right),
$$

where $v$ is a tangent vector to $M$, and the Gaussian curvature is

$$
K=-\left(\frac{4|d g / g|}{\left(|g|+|g|^{-1}\right)^{2}|d h|}\right)^{2} .
$$

If $(g, d h)$ is the Weierstrass data of a minimal surface $X: M \rightarrow \mathbb{R}^{3}$, then for each $\lambda>0$ the pair $(\lambda g, d h)$ satisfies condition $i)$ of Theorem 2.10 and the second equation in (5). The first equation in (5) holds for this new Weierstrass data if and only if $\int_{\gamma} g d h=\int_{\gamma} \frac{d h}{g}=0$ for all homology classes $\gamma$ in $M$, a condition which can be stated in terms of the notion of flux, which we now define. Given a minimal surface $M$ with Weierstrass data $(g, d h)$, the flux vector along a closed curve $\gamma \subset M$ is defined as

$$
F(\gamma)=\int_{\gamma} \operatorname{Rot}_{90^{\circ}}\left(\gamma^{\prime}\right)=\Im \int_{\gamma}\left(\frac{1}{2}\left(\frac{1}{g}-g\right), \frac{i}{2}\left(\frac{1}{g}+g\right), 1\right) d h \in \mathbb{R}^{3},
$$

where $\operatorname{Rot}_{90^{\circ}}$ denotes the rotation by angle $\pi / 2$ in the tangent plane of $M$ at any point, and $\Im$ stands for imaginary part.

Coming back to our Weierstrass data $(\lambda g, d h)$, the first equation in (5) holds for this new pair if and only if the flux of $M$ along $\gamma$ is a vertical vector for all closed curves $\gamma \subset M$. Thus, for a minimal surface $X$ with vertical flux and for $\lambda$ a positive real number, the Weierstrass data $(\lambda g, d h)$ produces a well-defined minimal surface $X_{\lambda}: M \rightarrow \mathbb{R}^{3}$. The family $\left\{X_{\lambda}\right\}_{\lambda}$ is a smooth deformation of $X_{1}=X$

\footnotetext{
${ }^{10}$ The reason for this name is that the failure of (5) implies that equation (4) only defines $X$ as an additively multivalued map. The translation vectors given by this multivaluation are called the periods of the pair $(g, d h)$.
} 
by minimal surfaces, called the López-Ros deformation 11 Clearly, the conformal structure, height differential and the set of points in $M$ with vertical normal vector are preserved throughout this deformation. Another important property of the López-Ros deformation is that if a component of a horizontal section of $X$ is convex, then the same property holds for the related component at the same height for any $X_{\lambda}, \lambda>0$. For details, see [104, 171].

2.3. Minimal surfaces with finite total curvature. Among the family of complete minimal surfaces in space, those with finite total curvature have been the most extensively studied. The principal reason for this is that they can be thought of as compact algebraic objects in a natural sense, which opens tremendously the number and depth of tools that can be applied to study these kinds of surfaces. We can illustrate this point of view with the example of the catenoid (see Section 2.5 for a computer image and discussion of this surface, as well as other examples of complete, embedded minimal surfaces of finite total curvature). The vertical catenoid $C$ is obtained as the surface of revolution of the graph of $\cosh x_{3}$ around the $x_{3}$ axis. It is straightforward to check that its Gauss map $N: C \rightarrow \mathbb{S}^{2}$ is a conformal diffeomorphism of $C$ with its image, which consists of $\mathbb{S}^{2}$ punctured in the north and south poles. Hence, the conformal compactification $\bar{C}$ of $C$ is conformally the sphere and the Gauss map extends holomorphically to the compactification $\bar{C}$. More generally, we have the following result.

Theorem 2.11 (Huber [87, Osserman 163]). Let $M \subset \mathbb{R}^{3}$ be a complete (oriented), immersed minimal surface with finite total curvature. Then,

i) $M$ is conformally a compact Riemann surface $\bar{M}$ with a finite number of points removed (called the ends of $M$ ).

ii) The Weierstrass data $(g, d h)$ extends meromorphically to $\bar{M}$. In particular, the total curvature of $M$ is a multiple of $-4 \pi$.

In this setting, the Gauss map $g$ has a well-defined finite degree on $\bar{M}$. A direct consequence of (3) is that the total curvature of an $M$ as in Theorem 2.11 is $-4 \pi$ times the degree of its Gauss map $g$. It turns out that this degree can be computed in terms of the genus of the compactification $\bar{M}$ and the number of ends, by means of the Jorge-Meeks formuld12 89. Rather than stating here this general formula for an immersed surface $M$ as in Theorem 2.11, we will emphasize the particular case when all the ends of $M$ are embedded:

$$
\operatorname{deg}(g)=\operatorname{genus}(\bar{M})+\#(\text { ends })-1 .
$$

The asymptotic behavior of a complete, embedded minimal surface in $\mathbb{R}^{3}$ with finite total curvature is well understood. Schoen 188 demonstrated that, after a rotation, each embedded end of a complete minimal surface with finite total curvature can be parameterized as a graph over the exterior of a disk in the $\left(x_{1}, x_{2}\right)$ plane with height function

$$
x_{3}\left(x_{1}, x_{2}\right)=a \log r+b+\frac{c_{1} x_{1}+c_{2} x_{2}}{r^{2}}+\mathcal{O}\left(r^{-2}\right),
$$

\footnotetext{
${ }^{11}$ This deformation is well known since López and Ros used it as the main ingredient in their proof of Theorem 3.2 below, although it had already been previously used by other authors; see Nayatani 153 .

${ }^{12}$ This is an application of the classical Gauss-Bonnet formula. Different authors, such as Gackstatter 64] and Osserman [163], have obtained related formulas.
} 
where $r=\sqrt{x_{1}^{2}+x_{2}^{2}}, a, b, c_{1}, c_{2} \in \mathbb{R}$ and $\mathcal{O}\left(r^{-2}\right)$ denotes a function such that $r^{2} \mathcal{O}\left(r^{-2}\right)$ is bounded as $r \rightarrow \infty$. The coefficient $a$ in (10) is called the logarithmic growth of the end. When $a \neq 0$, the end is called a catenoidal end; if $a=0$, we have a planar end. We use this language since a catenoidal end is asymptotic to one of the ends of a catenoid (see Section 2.5 for a description of the catenoid) and a planar end is asymptotic to the end of a plane. In particular, complete embedded minimal surfaces with finite total curvature are always proper; in fact, an elementary analysis of the asymptotic behavior shows that the equivalence between completeness and properness still holds for immersed minimal surfaces with finite total curvature.

As explained above, minimal surfaces with finite total curvature have been widely studied and their comprehension is the starting point to deal with more general questions about complete embedded minimal surfaces. In Section 2.5 we will briefly describe some examples in this family. Section 3 contains short explanations of some of the main results and constructions for finite total curvature minimal surfaces. Beyond these two short incursions, we will not develop extensively the theory of minimal surfaces with finite total curvature, since this is not the purpose this article, as explained in the Introduction. We refer the interested reader to the treatises by Hoffman and Karcher 74, López and Martín 103 and Pérez and Ros [171 for a more in-depth treatment of these special surfaces.

2.4. Periodic minimal surfaces. A properly embedded minimal surface $M \subset$ $\mathbb{R}^{3}$ is called singly, doubly, or triply periodic when it is invariant by an infinite group $G$ of isometries of $\mathbb{R}^{3}$ of rank 1,2,3 (respectively) that acts properly and discontinuously. Very often, it is useful to study such an $M$ as a minimal surface in the complete, flat three-manifold $\mathbb{R}^{3} / G$. Up to finite coverings and after composing by a fixed rotation in $\mathbb{R}^{3}$, these three-manifolds reduce to $\mathbb{R}^{3} / T, \mathbb{R}^{3} / S_{\theta}, \mathbb{T}^{2} \times \mathbb{R}$ and $\mathbb{T}^{3}$, where $T$ denotes a non-trivial translation, $S_{\theta}$ is the screw motion symmetry resulting from the composition of a rotation of angle $\theta$ around the $x_{3}$-axis with a translation in the direction of this axis, and $\mathbb{T}^{2}, \mathbb{T}^{3}$ are flat tori obtained as quotients of $\mathbb{R}^{2}, \mathbb{R}^{3}$ by 2 or 3 linearly independent translations, respectively.

Meeks and Rosenberg [131, 134, developed the theory of periodic minimal surfaces. For instance, they obtained in this setting similar conclusions to those given in Theorem 2.11. except that the Gauss map $g$ of a minimal surface in $\mathbb{R}^{3} / G$ is not necessarily well defined (the Gauss map $g$ does not descend to the quotient for surfaces in $\mathbb{R}^{3} / S_{\theta}, \theta \in(0,2 \pi)$, and in this case the role of $g$ is played by the well-defined meromorphic differential form $d g / g$ ). An important fact, due to Meeks and Rosenberg [131, 134, is that for properly embedded minimal surfaces in $\mathbb{R}^{3} / G$, $G \neq\{$ identity $\}$, the conditions of finite total curvature and finite topology are equivalent 13

Theorem 2.12 (Meeks, Rosenberg [137, 131, 134, 135]). A complete, embedded minimal surface in a non-simply connected, complete, flat three-manifold has finite topology if and only if it has finite total curvature. Furthermore, if $\Sigma$ denotes a compact surface of non-positive curvature and $M \subset \Sigma \times \mathbb{R}$ is a properly embedded minimal surface of finite genus, then $M$ has finite topology, finite index of stability and its finite total curvature is equal to $2 \pi$ times the Euler characteristic of $M$.

\footnotetext{
${ }^{13}$ This equivalence does not hold for properly embedded minimal surfaces in $\mathbb{R}^{3}$, as demonstrated by the helicoid.

${ }^{14}$ See Definition 2.23 for the definition of index of stability.
} 
The second statement in the above theorem was motivated by a result of Meeks [109, who proved that every properly embedded minimal surface in $\mathbb{T}^{2} \times \mathbb{R}$ has a finite number of ends; hence, in this setting, finite genus implies finite total curvature.

Meeks and Rosenberg [131, 134 also studied the asymptotic behavior of complete, embedded minimal surfaces with finite total curvature in $\mathbb{R}^{3} / G$. Under this condition, there are three possibilities for the ends of the quotient surface: all ends must be simultaneously asymptotic to planes (as in the Riemann minimal examples, see Section 2.5. such ends are called planar ends), to ends of helicoids (called helicoidal ends), or to flat annuli (as in the singly or doubly periodic Scherk minimal surfaces; for this reason, such ends are called Scherk-type ends).

2.5. Some interesting examples of complete minimal surfaces. We will now use the Weierstrass representation for introducing some of the most celebrated complete minimal surfaces. Throughout the presentation of these examples, we will freely use Collin's Theorem 1.3 and Colding-Minicozzi's Theorem 1.5

The plane. $M=\mathbb{C}, g(z)=1, d h=d z$. It is the only complete, flat minimal surface in $\mathbb{R}^{3}$.

The catenoid. $M=\mathbb{C}-\{0\}, g(z)=z, d h=\frac{d z}{z}$. In 1741, Euler 53 discovered that when a catenary $x_{1}=\cosh x_{3}$ is rotated around the $x_{3}$-axis, one obtains a surface which minimizes area among surfaces of revolution after prescribing boundary values for the generating curves. This surface was called the alysseid or since Plateau, the catenoid. In 1776, Meusnier verified that the catenoid is a solution of Lagrange's equation (11). This surface has genus zero, two ends and total curvature $-4 \pi$. Together with the plane, the catenoid is the only minimal surface of revolution (Bonnet [10]) and the unique complete, embedded minimal surface with genus zero, finite topology and more than one end (López and Ros [104, Collin 38, ColdingMinicozzi [37]). Also, the catenoid is characterized as being the unique complete, embedded minimal surface with finite topology and two ends (Schoen [188]). See Figure 1, left.

The helicoid. $M=\mathbb{C}, g(z)=e^{z}, d h=i d z$. This surface was first proved to be minimal by Meusnier in 1776 [146. When viewed in $\mathbb{R}^{3}$, the helicoid has genus zero, one end and infinite total curvature. Together with the plane, the helicoid is the only ruled minimal surface (Catalan [17) and is the unique simply connected, complete, embedded minimal surface (Meeks and Rosenberg [136, Colding and Minicozzi [37]). The vertical helicoid can also be viewed as a genus-zero surface with two ends in a quotient of $\mathbb{R}^{3}$ by a vertical translation or by a screw motion. See Figure 1, center. The catenoid and the helicoid are conjugate minimal surfaces, in the sense of the following definition.

Definition 2.13. Two minimal surfaces in $\mathbb{R}^{3}$ are said to be conjugate if the local coordinate functions of one of them are the harmonic conjugates of the local coordinate functions of the other one.

Note that in the case of the helicoid and catenoid, we consider the catenoid to be defined on its universal cover $e^{z}: \mathbb{C} \rightarrow \mathbb{C}-\{0\}$ in order for the harmonic conjugate of $x_{3}$ to be well defined. Equivalently, both surfaces share the Gauss map $e^{z}$ and their height differentials differ by multiplication by $i=\sqrt{-1}$. 

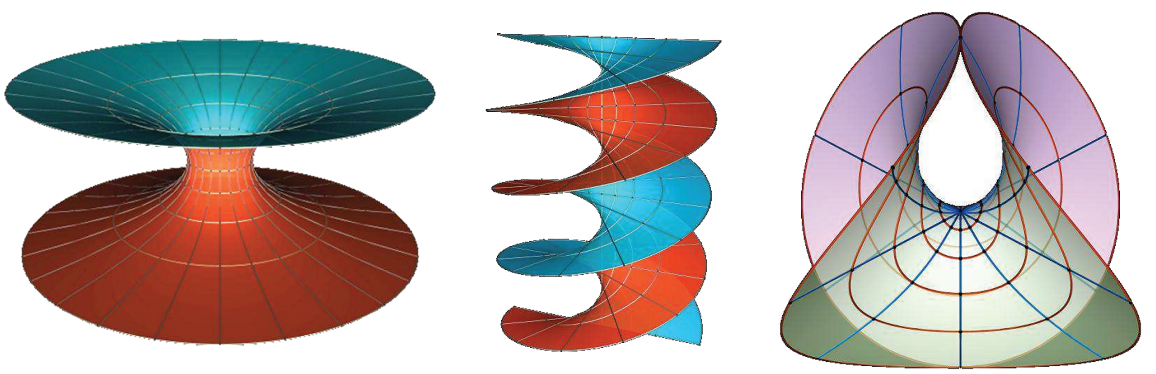

Figure 1. Left: The catenoid. Center: The helicoid. Right: The Enneper surface. Images courtesy of M. Weber.

The Enneper surface. $M=\mathbb{C}, g(z)=z, d h=z d z$. This surface was discovered by Enneper 52 in 1864, using his newly formulated analytic representation of minimal surfaces in terms of holomorphic data, equivalent to the Weierstrass representation 15 This surface is non-embedded, has genus zero, one end and total curvature $-4 \pi$. It contains two horizontal orthogonal lines (after cutting the surface along either of these lines, one divides it into two embedded pieces bounded by a line) and the entire surface has two vertical planes of reflective symmetry. See Figure 1, right. Every rotation of the coordinate $z$ around the origin in $\mathbb{C}$ is an (intrinsic) isometry of the Enneper surface, but most of these isometries do not extend to ambient isometries. The catenoid and Enneper's surface are the unique complete minimal surfaces in $\mathbb{R}^{3}$ with finite total curvature $-4 \pi$ (Osserman [163]). The implicit form of Enneper's surface is

$$
\left(\frac{y^{2}-x^{2}}{2 z}+\frac{2}{9} z^{2}+\frac{2}{3}\right)^{3}-6\left(\frac{y^{2}-x^{2}}{4 z}-\frac{1}{4}\left(x^{2}+y^{2}+\frac{8}{9} z^{2}\right)+\frac{2}{9}\right)^{2}=0 .
$$

The Meeks minimal Möbius strip. $M=\mathbb{C}-\{0\}, g(z)=z^{2}\left(\frac{z+1}{z-1}\right), d h=$ $i\left(\frac{z^{2}-1}{z^{2}}\right) d z$. Found by Meeks [106], the minimal surface defined by this Weierstrass data double covers a complete, immersed minimal surface $M_{1} \subset \mathbb{R}^{3}$ which is topologically a Möbius strip. This is the unique complete, minimally immersed surface in $\mathbb{R}^{3}$ of finite total curvature $-6 \pi$. It contains a unique closed geodesic which is a planar circle and also contains a line bisecting the circle; see Figure 2 left.

The bent helicoids. $M=\mathbb{C}-\{0\}, g(z)=-z \frac{z^{n}+i}{i z^{n}+i}, d h=\frac{z^{n}+z^{-n}}{2 z} d z$. Discovered by Meeks and Weber [140] and independently by Mira [149], these are complete, immersed minimal annuli $\widetilde{H}_{n} \subset \mathbb{R}^{3}$ with two non-embedded ends and finite total curvature; each of the surfaces $\widetilde{H}_{n}$ contains the unit circle $\mathbb{S}^{1}$ in the $\left(x_{1}, x_{2}\right)$-plane, and a neighborhood of $\mathbb{S}^{1}$ in $\widetilde{H}_{n}$ contains an embedded annulus $H_{n}$ which approximates, for $n$ large, a highly spinning helicoid whose usual straight axis has been periodically bent into the unit circle $\mathbb{S}^{1}$ (thus the name of bent helicoids); see Figure 2 right. Furthermore, the $H_{n}$ converge as $n \rightarrow \infty$ to the foliation of $\mathbb{R}^{3}$ minus the $x_{3}$-axis by vertical half-planes with boundary the $x_{3}$-axis, and with $\mathbb{S}^{1}$ as the

\footnotetext{
${ }^{15}$ For this reason, the Weierstrass representation is also referred to in the literature as the Enneper-Weierstrass representation.
} 

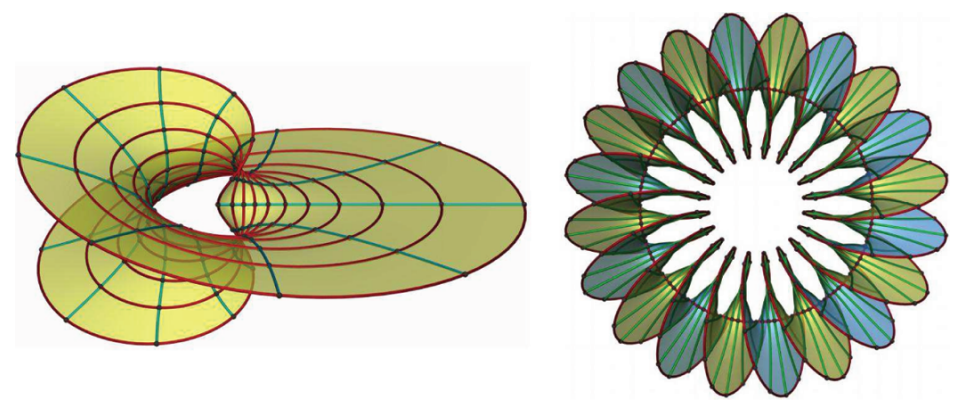

Figure 2. Left: The Meeks minimal Möbius strip. Right: A bent helicoid near the circle $\mathbb{S}^{1}$, which is viewed from above along the $x_{3}$-axis. Images courtesy of M. Weber.

singular set of $C^{1}$-convergence. The method applied by Meeks, Weber and Mira to find the bent helicoids is the classical Björling formula [160 with an orthogonal unit field along $\mathbb{S}^{1}$ that spins an arbitrary number $n$ of times around the circle. This construction also makes sense when $n$ is half an integer; in the case $n=\frac{1}{2}, \widetilde{H}_{1 / 2}$ is the double cover of the Meeks minimal Möbius strip described in the previous example. The bent helicoids $H_{n}$ play an important role in proving the converse of Meeks' $C^{1,1}$-Regularity Theorem (Theorem 9.6 below) for the singular set of convergence in a Colding-Minicozzi limit minimal lamination (for this converse, see Meeks and Weber [140]).

For the next group of examples, we need to introduce some notation. Given $k \in \mathbb{N}, k \geq 1$ and $a \in \mathbb{R}-\{0,-1\}$, we define the compact genus- $k$ surface $\bar{M}_{k, a}=$ $\left\{(z, w) \in(\mathbb{C} \cup\{\infty\})^{2} \mid w^{k+1}=\frac{(z+1)(z-a)}{z}\right\}$. Let $M_{k, a}=\bar{M}_{k, a}-\{(-1,0),(\infty, \infty)$, $(a, 0)\}$ and

$$
g_{k, a, m, A}(z, w)=A \frac{z w}{m z+1}, \quad d h_{k, a, m}=\frac{m z+1}{(z+1)(z-a)} d z
$$

where $A \in \mathbb{R}-\{0\}$. Given $k \in \mathbb{N}$ and $a \in(0, \infty)$, there exist $m=m(a) \in \mathbb{R}$ and $A=A(a) \in \mathbb{R}-\{0\}$ such that the pair $\left(g_{k, a, m(a), A(a)}, d h_{k, a, m(a)}\right)$ is the Weierstrass data of a well-defined minimal surface $X: M_{k, a} \rightarrow \mathbb{R}^{3}$ with genus $k$ and three ends (Hoffman, Karcher [74]). Moreover, $m(1)=0$ for any $k \in \mathbb{N}$. With this notation, we have the following examples.

The Costa torus. $M=M_{1,1}, g=g_{1,1,0, A(1)}, d h=d h_{1,1,0}$. Perhaps the most celebrated complete minimal surface in $\mathbb{R}^{3}$ since the classical examples from the nineteenth century 16 was discovered in 1982 by Costa [41, 42] This is a thrice punctured torus with total curvature $-12 \pi$, two catenoidal ends and one planar middle end. Costa 42 demonstrated the existence of this surface but only proved its embeddedness outside a ball in $\mathbb{R}^{3}$. Hoffman and Meeks [78] demonstrated its global embeddedness, thereby disproving a long-standing conjecture that the only

\footnotetext{
${ }^{16}$ It also should be mentioned that Chen and Gackstatter [19, 20 found in 1981 a complete minimal immersion (not embedded) of a once-punctured torus in $\mathbb{R}^{3}$, obtained by adding a handle to the Enneper surface. This surface was really a direct ancestor of the Costa torus and the Costa-Hoffman-Meeks surfaces; see Hoffman $[73$ for geometric and historical connections between these surfaces.
} 


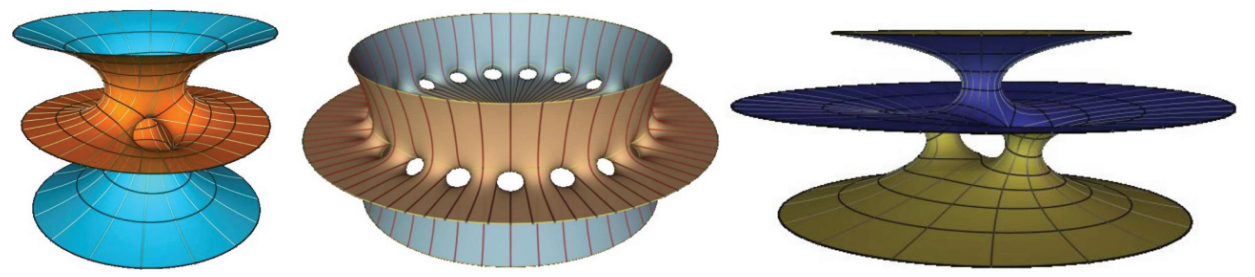

Figure 3. Left: The Costa torus. Center: A Costa-HoffmanMeeks surface of genus 20. Right: Deformed Costa. Images courtesy of M. Weber.

complete, embedded minimal surfaces in $\mathbb{R}^{3}$ of finite topological type are the plane, catenoid and helicoid. The Costa surface contains two horizontal straight lines $l_{1}, l_{2}$ that intersect orthogonally and has vertical planes of symmetry bisecting the right angles made by $l_{1}, l_{2}$; see Figure 3, left.

The Costa-Hoffman-Meeks surfaces. For any $k \geq 2$, take $M=M_{k, 1}, g=$ $g_{1,1,0, A(1)}, d h=d h_{1,1,0}$. These examples generalize the Costa torus (given by $k=1$ ), and are complete, embedded, genus $k$ minimal surfaces with two catenoidal ends and one planar middle end. Both existence and embeddedness were given by Hoffman and Meeks [79]. The symmetry group of the genus- $k$ example is generated by $180^{\circ}$-rotations about $k+1$ horizontal lines contained in the surface that intersect at a single point, together with the reflective symmetries in vertical planes that bisect those lines. As $k \rightarrow \infty$, suitable scalings of the $M_{k, 1}$ converge either to the singular configuration given by a vertical catenoid and a horizontal plane passing through its waist circle, or to the singly periodic Scherk minimal surface for $\theta=\pi / 2$ (Hoffman-Meeks [80). Both of these limits are suggested by Figure 3, center.

The deformation of the Costa torus. The Costa surface is defined on a square torus $M_{1,1}$, and admits a deformation (found by Hoffman and Meeks, unpublished) where the planar end becomes catenoidal. For any $a \in(0, \infty)$, take $M=M_{1, a}$ (which varies on arbitrary rectangular tori), $g=g_{1, a, m(a), A(a)}, d h=d h_{1, a, m(a)}$; see Figure 3. right. Thus, $a=1$ gives the Costa torus. The deformed surfaces are easily seen to be embedded for $a$ close to 1 . Hoffman and Karcher [74] proved the existence and embeddedness of these surfaces for all values of $a$; see also the survey by López and Martín [103]. Costa [43, 44] showed that any complete, embedded minimal torus with three ends must lie in this family.

The deformation of the Costa-Hoffman-Meeks surfaces. For any $k \geq 2$ and $a \in(0, \infty)$, take $M=M_{k, a}, g=g_{k, a, m(a), A(a)}, d h=d h_{k, a, m(a)}$. When $a=1$, we find the Costa-Hoffman-Meeks surface of genus $k$ and three ends. As in the case of genus 1, Hoffman and Meeks discovered this deformation for values of $a$ close to 1. A complete proof of existence and embeddedness for these surfaces is given in [74] by Hoffman and Karcher. These surfaces are conjectured to be the unique complete, embedded minimal surfaces in $\mathbb{R}^{3}$ with genus $k$ and three ends.

The genus-one helicoid. Now $M$ is conformally a certain rhombic torus $\mathbb{T}$ minus one point $E$. If we view $\mathbb{T}$ as a rhombus with edges identified in the usual manner, then $E$ corresponds to the vertices of the rhombus, and the diagonals of $\mathbb{T}$ are mapped into perpendicular straight lines contained in the surface, intersecting at a 

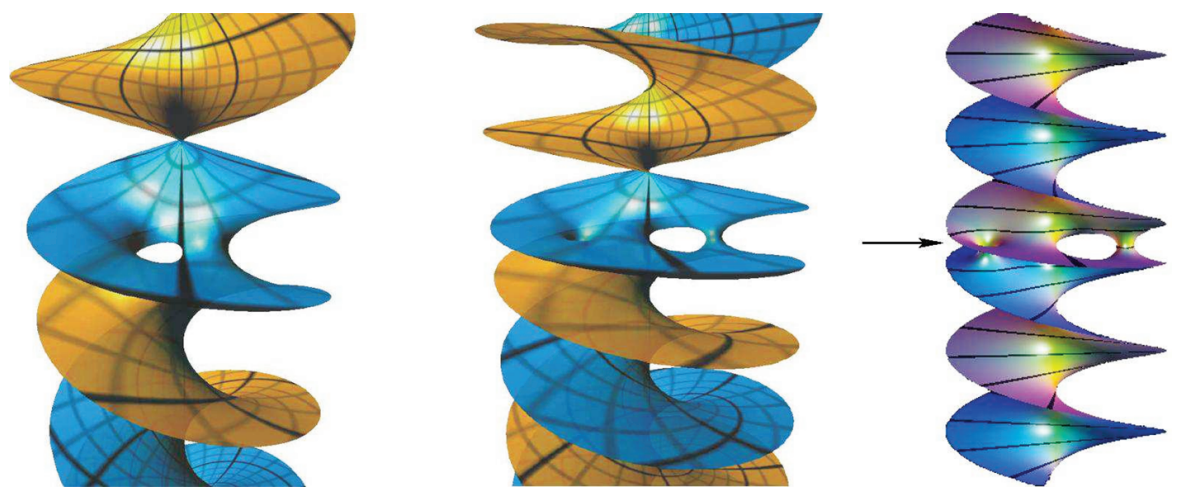

Figure 4. Left: The genus-one helicoid. Center and Right: Two views of the (possibly existing) genus-two helicoid. The arrow in the figure at the right points to the second handle. Images courtesy of M. Schmies (Left, Center) and M. Traizet (Right).

single point in space. The unique end of $M$ is asymptotic to a helicoid, so that one of the two lines contained in the surface is an axis (as in the genuine helicoid). The Gauss map $g$ is a meromorphic function on $\mathbb{T}-\{E\}$ with an essential singularity at $E$, and both $d g / g$ and $d h$ extend meromorphically to $\mathbb{T}$; see Figure 4 , left. This surface was discovered in 1993 by Hoffman, Karcher and Wei [75, 76]. Using flat structures, Hoffman, Weber and Wolf [83] proved the embeddedness of a genus one helicoid, obtained as a limit of singly periodic "genus one" helicoids invariant by screw motions of arbitrarily large angles 17 Later Hoffman and White 85] gave a variational proof of the existence of a genus-one helicoid. There is computational evidence pointing to the existence of a unique complete, embedded minimal surface in $\mathbb{R}^{3}$ with one helicoidal end for any positive genus (Traizet, unpublished, Bobenko [7, Bobenko and Schmies [8], Schmies [186]; also see Figure 4), but both the existence and the uniqueness questions remain unsolved (see Conjecture 11.6).

The singly periodic Scherk surfaces. $M=(\mathbb{C} \cup\{\infty\})-\left\{ \pm e^{ \pm i \theta / 2}\right\}, g(z)=z$, $d h=\frac{i z d z}{\Pi\left(z \pm e^{ \pm i \theta / 2}\right)}$, for fixed $\theta \in(0, \pi / 2]$. Discovered by Scherk [185] in 1835, these surfaces denoted by $\mathcal{S}_{\theta}$ form a 1-parameter family of complete, embedded, genuszero minimal surfaces in a quotient of $\mathbb{R}^{3}$ by a translation, and have four annular ends. Viewed in $\mathbb{R}^{3}$, each surface $\mathcal{S}_{\theta}$ is invariant under reflection in the $\left(x_{1}, x_{3}\right)$ and $\left(x_{2}, x_{3}\right)$-planes and in horizontal planes at integer heights, and can be thought of geometrically as a desingularization of two vertical planes forming an angle of $\theta$. The special case $\mathcal{S}_{\theta=\pi / 2}$ also contains pairs of orthogonal lines at planes of halfinteger heights and has an implicit equation $\sin z=\sinh x \sinh y$; see Figure 5, left. Together with the plane and catenoid, the surfaces $\mathcal{S}_{\theta}$ are conjectured to be the only connected, complete, immersed, minimal surfaces in $\mathbb{R}^{3}$ whose area in balls of radius $R$ is less than $2 \pi R^{2}$ (Conjecture 11.12). See the paper by Meeks and Wolf for

\footnotetext{
${ }^{17}$ These singly periodic "genus-one" helicoids have genus one in their quotient spaces and were discovered earlier by Hoffman, Karcher and Wei [77] (case of translation invariance) and by Hoffman and Wei 84 (case of screw-motion invariance).
} 

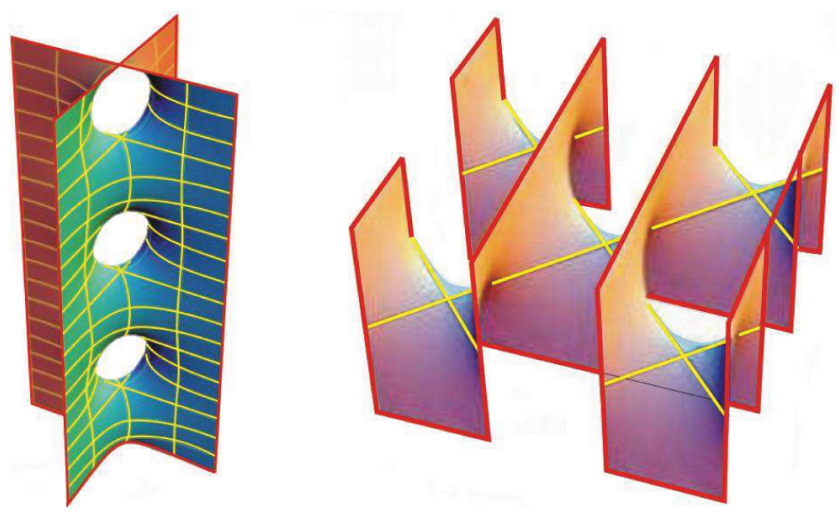

FIGURE 5. Singly periodic Scherk surface with angle $\theta=\frac{\pi}{2}$ (left), and its conjugate surface, the doubly periodic Scherk surface (right). Images courtesy of M. Weber.

a solution on this conjecture under the additional hypothesis of infinite symmetry, and also see [116.

The doubly periodic Scherk surfaces. $M=(\mathbb{C} \cup\{\infty\})-\left\{ \pm e^{ \pm i \theta / 2}\right\}, g(z)=$ $z, d h=\frac{z d z}{\prod\left(z \pm e^{ \pm i \theta / 2}\right)}$, where $\theta \in(0, \pi / 2]$ (the case $\theta=\frac{\pi}{2}$ has implicit equation $\left.e^{z} \cos y=\cos x\right)$. These surfaces, also discovered by Scherk [185] in 1835, are the conjugate surfaces of the singly periodic Scherk surfaces, and can be thought of geometrically as the desingularization of two families of equally spaced vertical parallel half-planes in opposite half-spaces, with the half-planes in the upper family making an angle of $\theta$ with the half-planes in the lower family; see Figure 5, right. These surfaces are doubly periodic with genus zero in their corresponding quotient $\mathbb{T}^{2} \times \mathbb{R}$, and were characterized by Lazard-Holly and Meeks 100 as being the unique properly embedded minimal surfaces with genus zero in any $\mathbb{T}^{2} \times \mathbb{R}$. It has been conjectured by Meeks, Pérez and Ros 122 that the singly and doubly periodic Scherk minimal surfaces are the only complete, embedded minimal surfaces in $\mathbb{R}^{3}$ whose Gauss maps miss four points on $\mathbb{S}^{2}$ (Conjecture 11.18). They also conjecture that the singly and doubly periodic Scherk minimal surfaces, together with the catenoid and helicoid, are the only complete, embedded minimal surfaces of negative curvature (Conjecture 11.17).

The Schwarz Primitive triply periodic surface. $M=\left\{(z, w) \in(\mathbb{C} \cup\{\infty\})^{2} \mid\right.$ $\left.w^{2}=z^{8}-14 z^{4}+1\right\}, g(z, w)=z, d h=\frac{z d z}{w}$. Discovered by Schwarz in the 1880 s, this minimal surface has a rank-three symmetry group and is invariant by translations in $\mathbb{Z}^{3}$. Such a structure, common to any triply periodic minimal surface (TPMS), is also known as a crystallographic cell or space tiling. Embedded TPMS divide $\mathbb{R}^{3}$ into two connected components (called labyrinths in crystallography), sharing $M$ as boundary (or interface) and interweaving each other. This property makes TPMS objects of interest to neighboring sciences, such as material sciences, crystallography, biology and others. For example, the interface between single calcite crystals and amorphous organic matter in the skeletal element in sea urchins is approximately described by the Schwarz Primitive surface [3, 50, 156. The piece 
of a TPMS that lies inside a crystallographic cell of the tiling is called a fundamental domain; see Figure 6 .

In the case of the Schwarz Primitive surface, one can choose a fundamental domain that intersects the faces of a cube in closed geodesics which are almost circles. In fact, the Schwarz Primitive surface has many more symmetries than those coming from the spatial tiling: some of them are produced by rotation around straight lines contained in the surface, which by the Schwarz reflection principl 18 divide the surface into congruent graphs with piecewise linear quadrilateral boundaries. Other interesting properties of this surface are that it divides space into two congruent three-dimensional regions (as do many other TPMSs), and the quotient surface in the three-torus associated to the above crystallographic cells is a compact hyperelliptic Riemann surface with genus three (in fact, any TPMS $\Sigma$ with genus three is hyperelliptic, since by application of the Gauss-Bonnet formula, the corresponding Gauss map $g: \Sigma \rightarrow \mathbb{S}^{2}$ is holomorphic with degree two). Its conjugate surface, also discovered by Schwarz, is another famous example of an embedded TPMS, called the Schwarz Diamond surface. In the 1960s, A. Schoen [187 made a surprising discovery: another associate surfact 19 of the Primitive and Diamond surface is an embedded TPMS, and he named this surface the Gyroid.

The Primitive, Diamond and Gyroid surfaces play important roles as surface interfaces in material sciences, in part since they are stable in their quotient tori under volume-preserving variations (see Ross [184). Furthermore, these surfaces have index of stability one, and Ros 180 has shown that any orientable, embedded minimal surface of index one in a flat three-torus must have genus three. He conjectures that the Primitive, Diamond and Gyroid are the unique index-one minimal surfaces in their tori, and furthermore, that any flat three-torus can have at most one embedded, orientable minimal surface of index one. We refer the interested reader to Karcher [93, 94, Meeks [108] and Ros [179] for further examples and properties of triply periodic minimal surfaces. We remark that Traizet [199] has shown that every flat three-torus contains an infinite number of embedded minimal surfaces of genus $g \geq 3$, which are prime in the sense that they do not descend to minimal surfaces in another three-torus.

The Riemann minimal examples. This is a one-parameter family of surfaces, defined in terms of a parameter $\lambda>0$. Let $M_{\lambda}=\left\{(z, w) \in(\mathbb{C} \cup\{\infty\})^{2} \mid w^{2}=\right.$ $z(z-\lambda)(\lambda z+1)\}-\{(0,0),(\infty, \infty)\}, g(z, w)=z, d h=A_{\lambda} \frac{d z}{w}$, for each $\lambda>0$, where $A_{\lambda}$ is a non-zero complex number satisfying $A_{\lambda}^{2} \in \mathbb{R}$. Discovered by Riemann (and posthumously published, Hattendorf and Riemann [175, 176]), these examples are invariant under reflection in the $\left(x_{1}, x_{3}\right)$-plane and by a translation $T_{\lambda}$. The induced surfaces $M_{\lambda} / T_{\lambda}$ in the quotient spaces $\mathbb{R}^{3} / T_{\lambda}$ have genus one and two planar ends; see 124 for a more precise description. The Riemann minimal examples have the amazing property that every horizontal plane intersects each of these surfaces in a circle or in a line; see Figure 7 . The conjugate minimal surface of the

\footnotetext{
${ }^{18} \mathrm{~A}$ minimal surface that contains a straight line segment $l$ (resp. a planar geodesic) is invariant under the rotation of angle $180^{\circ}$ about $l$ (resp. under reflection in the plane that contains $l$ ). Both assertions follow directly from the general Schwarz reflection principle for harmonic functions.

${ }^{19}$ The family of associate surfaces of a simply connected minimal surface with Weierstrass data $(g, d h)$ are those with the same Gauss map and height differential $e^{i \theta} d h, \theta \in[0,2 \pi)$. In particular, the case $\theta=\pi / 2$ is the conjugate surface. This notion can be generalized to non-simply connected surfaces, although in that case the associate surfaces may have periods.
} 

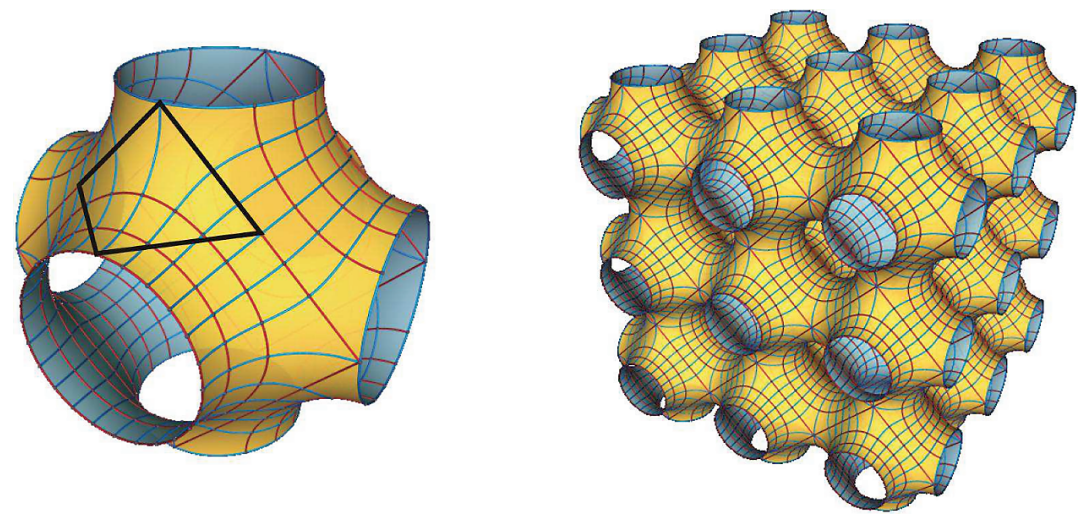

Figure 6. Left: A fundamental domain of the Schwarz P-surface, with a graphical quadrilateral. Right: A bigger piece of the corresponding space tiling. Images courtesy of M. Weber.
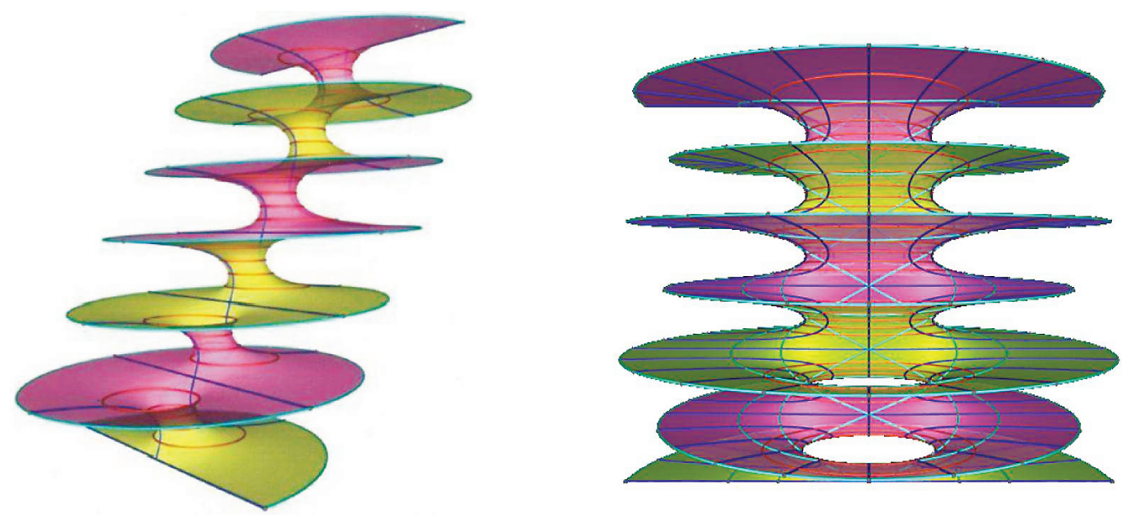

Figure 7. Two Riemann minimal examples (for different values of the parameter $\lambda$ ). Images courtesy of M. Weber.

Riemann minimal example for a given $\lambda>0$ is the Riemann minimal example for the parameter value $1 / \lambda$ (the case $\lambda=1$ gives the only self-conjugate surface in the family). Meeks, Pérez and Ros [124] showed that these surfaces are the only properly embedded minimal surfaces in $\mathbb{R}^{3}$ of genus zero and infinite topology (see Section 10).

The KMR doubly periodic tori. Given $(\theta, \alpha, \beta) \in\left(0, \frac{\pi}{2}\right) \times\left[0, \frac{\pi}{2}\right] \times\left[0, \frac{\pi}{2}\right]$ with $(\alpha, \beta) \neq(0, \theta)$, we consider the rectangular torus $\Sigma_{\theta}=\left\{(z, w) \in(\mathbb{C} \cup\{\infty\})^{2} \mid w^{2}=\right.$ $\left.\left(z^{2}+\lambda^{2}\right)\left(z^{2}+\lambda^{-2}\right)\right\}$, where $\lambda=\cot \frac{\theta}{2}$. Then $M=\Sigma_{\theta}-g^{-1}(\{0, \infty\})$,

$$
g(z, w)=\frac{z\left(i \cos \left(\frac{\alpha-\beta}{2}\right)+\cos \left(\frac{\alpha+\beta}{2}\right)\right)+\sin \left(\frac{\alpha-\beta}{2}\right)+i \sin \left(\frac{\alpha+\beta}{2}\right)}{\cos \left(\frac{\alpha-\beta}{2}\right)+i \cos \left(\frac{\alpha+\beta}{2}\right)-z\left(i \sin \left(\frac{\alpha-\beta}{2}\right)+\sin \left(\frac{\alpha+\beta}{2}\right)\right)}, \quad \text { and } \quad d h=\mu \frac{d z}{w},
$$

where $\mu=1$ or $\sqrt{-1}$. This Weierstrass data gives rise to a three-dimensional family of doubly periodic minimal surfaces in $\mathbb{R}^{3}$, that in the smallest quotient in some 

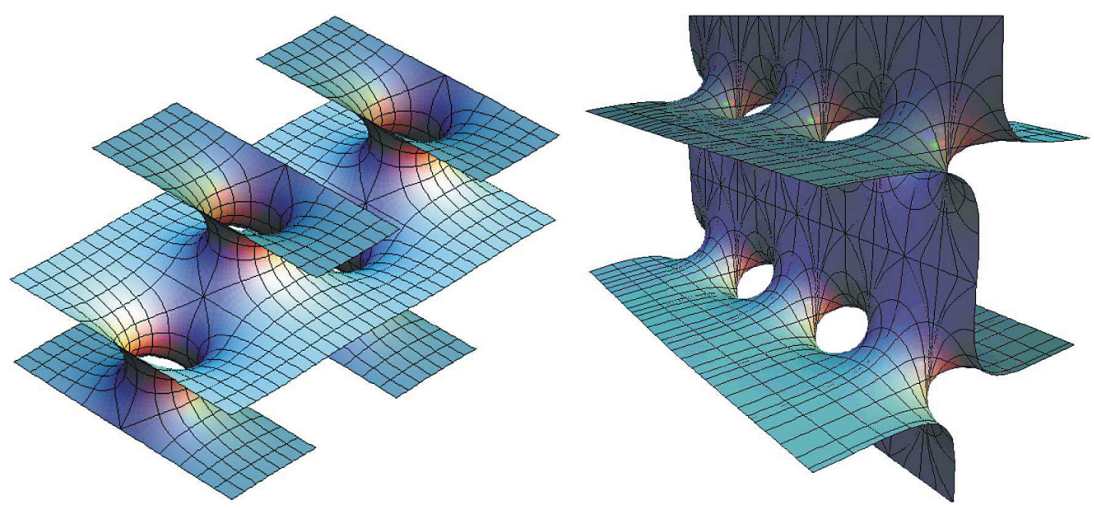

Figure 8. Two examples of doubly periodic KMR surfaces. Images taken from the 3D-XplorMath Surface Gallery.

$\mathbb{T}^{2} \times \mathbb{R}$ have four parallel Scherk-type ends and total curvature $-8 \pi$. Furthermore, the conjugate surface of any KMR surface also lies in this family. The first KMR surfaces were found by Karcher 92 in 1988 (he found three subfamilies, each one with dimension one, and named them toroidal half-plane layers). One year later, Meeks and Rosenberg 131 found examples of the same type as Karcher's, although the different nature of their approach made it unclear what the relationship was between their examples and those by Karcher. In 2005, Pérez, Rodríguez and Traizet 169. gave a general construction that produces all possible complete, embedded minimal tori with parallel ends in any $\mathbb{T}^{2} \times \mathbb{R}$ and proved that this moduli space reduces to the three-dimensional family of surfaces defined by the Weierstrass data given above; see Figure 8. It is conjectured that the only complete, embedded minimal surfaces in $\mathbb{R}^{3}$ whose Gauss map misses exactly 2 points on $\mathbb{S}^{2}$ are the catenoid, helicoid, Riemann examples, and these KMR examples.

The singly periodic Callahan-Hoffman-Meeks surfaces. In 1989, Callahan, Hoffman and Meeks 15 generalized the Riemann minimal examples by constructing for any integer $k \geq 1$ a singly periodic, properly embedded minimal surface $M_{k} \subset \mathbb{R}^{3}$ with infinite genus and an infinite number of horizontal planar ends at integer heights, invariant under the orientation-preserving translation by vector $T=(0,0,2)$, such that $M_{k} / T$ has genus $2 k+1$ and two ends. They not only produced the Weierstrass data of the surface (see [15] for details), but also gave an alternative method for finding this surface, based on blowing up a limiting singularity of a sequence of compact minimal annuli with boundaries. This rescaling process was a prelude to the crucial role that rescaling methods play nowadays in minimal surface theory and that we will treat in some detail in this survey. Other properties of the surfaces $M_{k}$ are the following ones.

(1) Every horizontal plane at a non-integer height intersects $M_{k}$ in a simple closed curve.

(2) Every horizontal plane at an integer height intersects $M_{k}$ in $k+1$ straight lines that meet at equal angles along the $x_{3}$-axis.

(3) Every horizontal plane at half-integer heights $n+\frac{1}{2}$ is a plane of symmetry of $M_{k}$, and any vertical plane whose reflection leaves invariant the horizontal 

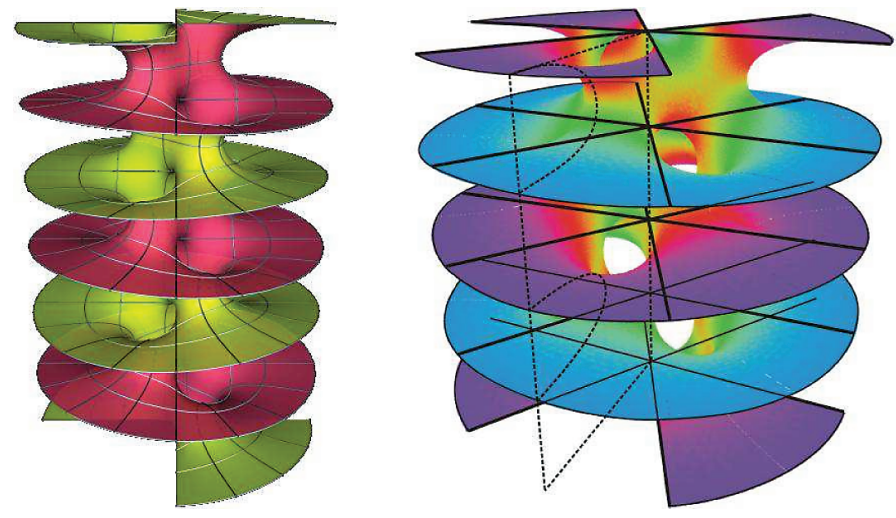

Figure 9. Callahan-Hoffman-Meeks surfaces $M_{k}$, for $k=1$ (left, image courtesy of M. Weber) and $k=2$ (right, from the Scientific Graphics Project at http://www.msri.org/about/ $\mathrm{sgp} / \mathrm{SGP} /$ index.html). The dotted lines correspond to a vertical plane of symmetry of $M_{2}$.

lines on $M_{k}$ described in item 2 is also a plane of symmetry. For pictures of $M_{k}$ with $k=1,2$, see Figure 9 .

2.6. Monotonicity formula and classical maximum principles. As we will see in Section 7.3 the conformal type of a minimal surface in $\mathbb{R}^{3}$ is strongly influenced by its area growth in extrinsic balls. The first result along these lines comes from the coarea formula applied to the distance function to a given point $p \in \mathbb{R}^{3}$. The following statement of the coarea formula appears in [18]; see [56] for a more general version.

Proposition 2.14 (Coarea Formula). Let $\Omega$ be a domain with compact closure in a Riemannian n-manifold $M$ and $f: \bar{\Omega} \rightarrow \mathbb{R}$ a function in $C^{0}(\bar{\Omega}) \cap C^{\infty}(\Omega)$ with $\left.f\right|_{\partial \Omega}=0$. For any regular value $t$ of $|f|$, we let $\Gamma(t)=|f|^{-1}(t)$ and let $A(t)$ denote the $(n-1)$-dimensional area of $\Gamma(t)$. Then, for any function $\phi \in L^{1}(\Omega)$, we have

$$
\int_{\Omega} \phi|\nabla f| d V=\int_{0}^{\infty}\left(\int_{\Gamma(t)} \phi d A_{t}\right) d t,
$$

where $\nabla f$ is the gradient of $f$ in $M$ and $d V, d A_{t}$ are respectively the volume elements in $M$ and $\Gamma(t)$.

Theorem 2.15 (Monotonicity Formula 25, 96). Let $X: M \rightarrow \mathbb{R}^{3}$ be a connected, properly immersed minimal surface. Given $p \in \mathbb{R}^{3}$, let $A(R)$ be the area of the portion of $X(M)$ inside a ball of radius $R>0$ centered at $p$. Then, $A(R) R^{-2}$ is non-decreasing. In particular, $\lim _{R \rightarrow \infty} A(R) R^{-2} \geq \pi$ with equality if and only if $M$ is a plane.

One of the consequences of the fact that minimal surfaces can be viewed locally as solutions of the partial differential equation (11) is that they satisfy certain maximum principles. We will state them for minimal surfaces in $\mathbb{R}^{3}$, but they also hold when the ambient space is any Riemannian three-manifold. 
Theorem 2.16 (Interior Maximum Principle [188]). Let $M_{1}, M_{2}$ be connected minimal surfaces in $\mathbb{R}^{3}$ and $p$ an interior point to both surfaces, such that $T_{p} M_{1}=$ $T_{p} M_{2}=\left\{x_{3}=0\right\}$. If $M_{1}, M_{2}$ are locally expressed as the graphs of functions $u_{1}, u_{2}$ around $p$ and $u_{1} \leq u_{2}$ in a neighborhood of $p$, then $M_{1}=M_{2}$ in a neighborhood of $p$.

A beautiful application of Theorem 2.16 is the following result by Hoffman and Meeks.

Theorem 2.17 (Half-space Theorem [82]). Let $M \subset \mathbb{R}^{3}$ be a proper, connected, possibly branched, non-planar minimal surface without boundary. Then $M$ cannot be contained in a half-space.

More generally, one has the following result of Meeks and Rosenberg based on earlier partial results in [22, 82, 98, 132, 192].

Theorem 2.18 (Maximum Principle at Infinity [138]). Let $M_{1}, M_{2} \subset N$ be disjoint, connected, properly immersed minimal surfaces with (possibly empty) boundary in a complete flat three-manifold $N$.

i) If $\partial M_{1} \neq \varnothing$ or $\partial M_{2} \neq \varnothing$, then after possibly reindexing, the distance between $M_{1}$ and $M_{2}$ (as subsets of $N$ ) is equal to $\inf \left\{\operatorname{dist}(p, q) \mid p \in \partial M_{1}, q \in\right.$ $\left.M_{2}\right\}$.

ii) If $\partial M_{1}=\partial M_{2}=\varnothing$, then $M_{1}$ and $M_{2}$ are flat.

We now come to a beautiful and deep application of the general maximum principle at infinity. The next corollary appears in 138 and a slightly weaker variant of it in Soret [192.

Corollary 2.19 (Regular Neighborhood Theorem). Suppose $M \subset N$ is a properly embedded minimal surface in a complete flat three-manifold $N$, with the absolute value of the Gaussian curvature of $M$ at most 1 . Let $N_{1}(M)$ be the open unit interval bundle of the normal bundle of $M$ given by the normal vectors of length strictly less than 1 . Then, the corresponding exponential map exp: $N_{1}(M) \rightarrow N$ is a smooth embedding. In particular:

1. $M$ has an open, embedded tubular neighborhood of radius 1.

2. There exists a constant $C>0$ such that for all balls $B \subset N$ of radius 1 , the area of $M \cap B$ is at most $C$ times the volume of $B$.

2.7. Ends of properly embedded minimal surfaces. One of the fundamental problems in classical minimal surface theory is to describe the behavior of a properly embedded minimal surface $M \subset \mathbb{R}^{3}$ outside a large compact set in space. This problem is well understood if $M$ has finite total curvature (see Theorem 2.11), because in this case, each of the ends of $M$ is asymptotic to an end of a plane or a catenoid. Theorem 1.4 states that if $M$ has finite topology but infinite total curvature (thus $M$ has exactly one end by Collin's Theorem 1.3), then $M$ is asymptotic to a helicoid. More complicated asymptotic behaviors can be found in periodic minimal surfaces in $\mathbb{R}^{3}$, although this asymptotic behavior is completely understood when the periodic minimal surface has finite topology in the corresponding quotient ambient space (thus the quotient surface has finite total curvature by Theorem 2.12); in this setting, only planar, helicoidal or Scherk-type ends can occur; see Section 2.4 . 
We next consider the question of understanding the asymptotics of a general properly embedded minimal surface in $\mathbb{R}^{3}$. A crucial point is the notion of topological end, which we now explain. Let $M$ be a non-compact connected manifold. We define an equivalence relation in the set $\mathcal{A}=\{\alpha:[0, \infty) \rightarrow M \mid \alpha$ is a proper arc $\}$, by setting $\alpha_{1} \sim \alpha_{2}$ if for every compact set $C \subset M, \alpha_{1}, \alpha_{2}$ lie eventually 20 in the same component of $M-C$.

Definition 2.20. Each equivalence class in $\mathcal{E}(M)=\mathcal{A} / \sim$ is called an end of $M$. If $e \in \mathcal{E}(M), \alpha \in e$ is a representative proper arc and $\Omega \subset M$ is a proper subdomain with compact boundary such that $\alpha \subset \Omega$, then we say that the domain $\Omega$ represents the end $e$.

The space $\mathcal{E}(M)$ has the following natural Hausdorff topology. For each proper domain $\Omega \subset M$ with compact boundary, we define the basis open set $B(\Omega) \subset \mathcal{E}(M)$ to be those equivalence classes in $\mathcal{E}(M)$ which have representatives contained in $\Omega$. With this topology, $\mathcal{E}(M)$ is a totally disconnected compact space which embeds topologically as a subspace of $[0,1] \subset \mathbb{R}$. On pages 288-289 of [117], we gave a short proof of this embedding result for $\mathcal{E}(M)$, which works even in the more general case where $M$ is a manifold or a finite-dimensional simplicial complex. In the case that $M$ is a properly embedded minimal surface in $\mathbb{R}^{3}$ with more than one end, there is also a natural topological embedding of $\mathcal{E}(M)$ into $[0,1]$ which uses the relative heights of the ends of $M$; see the Ordering Theorem 6.2 .

Definition 2.21. Any isolated point $e \in \mathcal{E}(M)$ is called a simple end of $M$. If $e \in \mathcal{E}(M)$ is not a simple end (equivalently, if it is a limit point of $\mathcal{E}(M) \subset[0,1]$ ), we will call it a limit end of $M$.

When $M$ has dimension 2 , then an elementary topological analysis using compact exhaustions shows that an end $e \in \mathcal{E}(M)$ is simple if and only if it can be represented by a proper subdomain $\Omega \subset M$ with compact boundary which is homeomorphic to one of the following models:

(a) $\mathbb{S}^{1} \times[0, \infty)($ this case is called an annular end $)$.

(b) $\mathbb{S}^{1} \times[0, \infty)$ connected sum with an infinite number of tori where the $n$th connected sum occurs at the point $(1, n) \in \mathbb{S}^{1} \times[0, \infty), n \in \mathbb{N}$ (this case is called a simple end of infinite genus).

For limit ends there are similar notions: a limit end $e \in \mathcal{E}(M)$ is said to have genus zero if it can be represented by a proper subdomain $\Omega \subset M$ with compact boundary and genus zero. If a limit end $e$ does not have genus zero, then we say that it has infinite genus; in this case, every proper subdomain with compact boundary representing $e$ has infinite genus.

We will devote Section 6 to the Ordering Theorem for ends of properly embedded minimal surfaces in $\mathbb{R}^{3}$; this theorem is the starting point for the theory of properly embedded minimal surfaces with more than one end. Concerning one-ended minimal surfaces, the classical example in this family is the helicoid. Also, one has the newer examples of helicoids with handles (rigorously proven to exist only for the case of one handle); see Figure 4. Theoretically, Theorem 1.4 insures that any non-planar, properly embedded, one-ended, minimal surface with finite topology must be necessarily asymptotic to a helicoid with finitely many handles, and it can

\footnotetext{
${ }^{20}$ Throughout the paper, the word eventually for proper arcs means outside a compact subset of the parameter domain $[0, \infty)$.
} 
be described analytically by meromorphic data $(d g / g, d h)$ on a compact Riemann surface by means of the classical Weierstrass representation. Regarding one-ended surfaces with infinite topology, Callahan, Hoffman and Meeks [16] showed that any non-flat, doubly or triply periodic minimal surface properly embedded in $\mathbb{R}^{3}$ must have infinite genus and only one end.

2.8. Second variation of area, index of stability and Jacobi functions. Let $M \subset \mathbb{R}^{3}$ be an oriented minimal surface and $\Omega \subset M$ a subdomain with compact closure. Any smooth normal deformation of the inclusion $X: M \rightarrow \mathbb{R}^{3}$ which is compactly supported in $\Omega$ can be written as $X+t u N$, where $N$ is the Gauss map of $M$ and $u \in C_{0}^{\infty}(\Omega)$. By equation (2), the area functional $A=A(t)$ for this deformation has $A^{\prime}(0)=0$. The second variation of area can be easily shown to be (see [160])

$$
A^{\prime \prime}(0)=-\int_{\Omega} u(\Delta u-2 K u) d A,
$$

where $K$ is the Gaussian curvature function of $M$ and $\Delta$ its Laplace operator. Formula (11) can be viewed as the bilinear form associated to the linear elliptic $L^{2}$-self-adjoint operator $L=\Delta-2 K=\Delta+|\nabla N|^{2}$, which is usually called the Jacobi operator or stability operator.

Definition 2.22. A $C^{2}$-function $u: M \rightarrow \mathbb{R}$ satisfying $\Delta u-2 K u=0$ on $M$ is called a Jacobi function. We will let $\mathcal{J}(M)$ denote the linear space of Jacobi functions on $M$.

Classical elliptic theory implies that given a subdomain $\Omega \subset M$ with compact closure, the Dirichlet problem for the Jacobi operator in $\Omega$ has an infinite discrete spectrum $\left\{\lambda_{k}\right\}_{k \in \mathbb{N} \cup\{0\}}$ of eigenvalues with $\lambda_{k} \nearrow+\infty$ as $k$ goes to infinity, and each eigenspace is a finite-dimensional linear subspace of $C^{\infty}(\Omega) \cap H_{0}^{1}(\Omega)$, where $H_{0}^{1}(\Omega)$ denotes the usual Sobolev space of $L^{2}$-functions with $L^{2}$ weak partial derivatives and trace zero. Since any normal variation by minimal surfaces has vanishing second derivative of the area functional, it follows that the normal parts of variational fields coming from Killing or dilatation vector fields of $\mathbb{R}^{3}$ produce elements in $\mathcal{J}(M)$. For instance, when the Killing field is a translation by a vector $v \in \mathbb{R}^{3}$, then the corresponding Jacobi function is $\langle N, v\rangle$ (called a linear Jacobi function). Similarly, rotations around an axis of direction $v \in \mathbb{R}^{3}$ produce the Jacobi function $\operatorname{det}(p, N, v)$ (here $p$ denotes the position vector) and homotheties give rise to the support function $\langle p, N\rangle \in \mathcal{J}(M)$.

A particularly interesting Jacobi function, which can be defined when the minimal surface is transverse to a family of horizontal planes, is the Shiffman function, which is proportional to the derivative of the curvature of each planar section with respect to a parameter of such a section (this parameter is not the arclength of the section; see equation (13)). Thus, the condition of vanishing identically for the Shiffman function means that the surface is foliated by planar curves of constant curvature, i.e., lines or circles. This argument can be used to prove that every compact minimal annulus $A$ in $\mathbb{R}^{3}$ whose boundary consists of circles in parallel planes $\Pi_{1}, \Pi_{2}$, is foliated by circles which lie in planes parallel to $\Pi_{1}$ (see Shiffman [190]). By classical results of Riemann 175, 176, it follows that such an annulus $A$ must be contained in a catenoid or in one of the Riemann minimal examples discussed in Section 2.5. The Shiffman function and its connection with the Korteweg-de 
Vries equation plays a fundamental role in the characterization by Meeks, Pérez and Ros 124] of the Riemann minimal examples as the only properly embedded minimal surfaces in $\mathbb{R}^{3}$ with their topology (see Theorem 1.2 and Section 10.3). For more details about the Shiffman function, see [54, 55, 117, 164, 167.

Definition 2.23. Let $\Omega \subset M$ be an orientable subdomain with compact closure. The index of stability of $\Omega$ is the number of negative eigenvalues of the Dirichlet problem associated to $L$ in $\Omega$. The nullity of $\Omega$ is the dimension of $\mathcal{J}(\Omega) \cap H_{0}^{1}(\Omega)$. $\Omega$ is called stable if its index of stability is zero, and strictly stable if both its index and nullity are zero.

Elliptic theory also implies that $\Omega$ is strictly stable provided that it is sufficiently small, which justifies the Definition 2.5 of minimal surface as a local minimum of area. Another consequence of elliptic theory is that $\Omega$ is stable and orientable if and only if it carries a positive Jacobi function. Since the Gauss map $N$ of a graph defined on a domain in a plane $\Pi$ has image set contained in an open half-sphere, the inner product of $N$ with the unit normal to $\Pi$ provides a positive Jacobi function, from where we conclude that any minimal graph is stable. Stability makes sense for non-compact minimal surfaces, as we next explain.

Definition 2.24. A minimal surface $M \subset \mathbb{R}^{3}$ is called stable if any subdomain $\Omega \subset M$ with compact closure is stable. For orientable minimal surfaces, stability is equivalent to the existence of a positive Jacobi function on $M$ (Proposition 1 in Fischer-Colbrie [59]). $M$ is said to have finite index if outside of a compact subset it is stable. The index of stability of $M$ is the supremum of the indices of stability of subdomains with compact closure in $M$.

By definition, stable surfaces have index zero. The following theorem explains how restrictive is the property of stability for complete minimal surfaces. It was proved independently by Fischer-Colbrie and Schoen [60, do Carmo and Peng [49], and Pogorelov [174] for orientable surfaces. Later, Ros [180] proved that a complete, non-orientable minimal surface in $\mathbb{R}^{3}$ is never stable.

Theorem 2.25. If $M \subset \mathbb{R}^{3}$ is a complete, immersed, stable minimal surface, then $M$ is a plane.

A short elementary proof of Theorem 2.25 in the orientable case is given in Section 4 of [117] and also in [114; also see the proof of Lemma 10.5 below.

A crucial fact in minimal surface theory is that stable, minimally immersed surfaces with boundary in $\mathbb{R}^{3}$ have curvature estimates up to their boundary. These curvature estimates were first obtained by Schoen for two-sided surfaces in homogeneously regular three-manifolds (see Definition 2.26 below) and later improved by Ros to the one-sided case, and are a simple consequence of Theorem 2.25 after a rescaling argument.

Definition 2.26. A Riemannian three-manifold $N$ is homogeneously regular if there exists an $\varepsilon>0$ such that $\varepsilon$-balls in $N$ are uniformly close to $\varepsilon$-balls in $\mathbb{R}^{3}$ in the $C^{2}$-norm. In particular, if $N$ is compact, then $N$ is homogeneously regular.

Theorem 2.27 (Schoen 188, Ros [180]). For any homogeneously regular threemanifold $N$ there exists a universal constant $c>0$ such that for any stable, minimally immersed surfac ${ }^{21} M$ in $N$, the absolute value $|K|$ of the Gaussian curvature

\footnotetext{
${ }^{21}$ For a two-sided minimal surface $M$ in a general three-manifold $N$ with unit normal vector $\nu: M \rightarrow T N$, the second derivative of the area functional along a compactly supported normal
} 
of $M$ satisfies

$$
|K(p)| \operatorname{dist}_{N}(p, \partial M)^{2} \leq c \quad \text { for all } p \in M
$$

where dist $_{N}$ denotes distance in $N$ and $\partial M$ is the boundary of $M$.

We remark that Rosenberg, Souam and Toubiana [183] have obtained a version of Theorem 2.27 valid in the two-sided case when the ambient three-manifold has a bound on its sectional curvature.

If we weaken the stability hypothesis in Theorem 2.25 to finite index of stability and we allow compact boundary, then completeness and orientability also lead to a well-known family of minimal surfaces.

Theorem 2.28 (Fischer-Colbrie [59]). Let $M \subset \mathbb{R}^{3}$ be a complete, orientable, minimally immersed surface in $\mathbb{R}^{3}$, with possibly empty compact boundary. Then $M$ has finite index of stability if and only if it has finite total curvature.

A similar result to Theorem 2.28 for non-orientable, complete minimal surfaces is not known to hold; see Conjecture 11.24.

By the conformal invariance of the Dirichlet integral, both the index and nullity of the Jacobi operator $L=\Delta+|\nabla N|^{2}$ remain constant under a conformal change of metric. Recall that the Huber-Osserman Theorem 2.11 asserts that every complete, orientable, immersed minimal surface $M \subset \mathbb{R}^{3}$ with finite total curvature is conformally equivalent to a finitely punctured compact Riemann surface $\bar{M}$, and the Gauss map $N$ of $M$ extends meromorphically to $\bar{M}$. In this case, it can be shown (Pérez and Ros 170]) that there exists a smooth metric $\overline{d s}^{2}$ on $\bar{M}$ such that the metric $d s^{2}$ on $M$ induced by the inner product of $\mathbb{R}^{3}$ can be expressed as $d s^{2}=\mu \overline{d s}^{2}$, where $\mu$ is a positive smooth function which blows up at the ends of $M$. Furthermore, both the index and nullity of $L$ can be computed as the index and nullity of the operator $\bar{L}=\bar{\Delta}+|\bar{\nabla} N|^{2}$ on $\bar{M}$, where a bar means that the corresponding object refers to $\overline{d s}^{2}$. Note that $\bar{L}$ is nothing more than the classical Schrödinger operator associated to the meromorphic extension of $N$ to $\bar{M}$. The subspace $\mathcal{K}(M)$ of bounded Jacobi functions on $M$ can be identified with the eigenspace associated to the eigenvalue 0 of the operator $\bar{L}$. Inside $\mathcal{K}(M)$, we have the subspace of linear functions $\mathcal{L}(M)=\left\{\langle N, v\rangle \mid v \in \mathbb{R}^{3}\right\}$ (which are the normal parts of variational fields of deformations by translations). If additionally all the ends of $M$ are parallel 22 say horizontal, then the function $\operatorname{det}\left(p, N, e_{3}\right) \in \mathcal{K}(M)$, where $e_{3}=(0,0,1)$, is a bounded Jacobi function (it is the normal part of the variational field of the deformation of $M$ by rotations around the $x_{3}$-axis). In particular, $\mathcal{K}(M)$ has dimension at least 4 for any complete, embedded minimal surface of finite total curvature in $\mathbb{R}^{3}$ (except for the catenoid and the plane, where $\operatorname{det}\left(p, N, e_{3}\right)$ vanishes).

Montiel and Ros found a beautiful relationship between bounded Jacobi functions and branched minimal immersions with prescribed Gauss map. For a complete

variation with variational field $u \nu$, is $A^{\prime \prime}(0)=-\int_{M} u\left(\Delta u+\left(\operatorname{Ric}(\nu)+|\sigma|^{2}\right) u\right) d A$, where Ric denotes the Ricci curvature of $N$ and $|\sigma|^{2}$ is the squared length of the second fundamental form of $M$ in $N$. $M$ is called stable if $A^{\prime \prime}(0) \geq 0$ for all $u \in C_{0}^{\infty}(M)$, and unstable otherwise. If $M$ is one-sided (i.e., has a non-trivial normal bundle), then stability imposes $A^{\prime \prime}(0) \geq 0$ on the two-sided covering $\widetilde{M}$ of $M$, for all functions $u \in C_{0}^{\infty}(\widetilde{M})$ which anti-commute with the natural $2: 1$ covering map $\widetilde{M} \rightarrow M$. When the ambient manifold $N$ is orientable, two-sidedness is equivalent to orientability of $M$.

${ }^{22}$ This occurs, for instance, when $M$ is embedded. 
minimal surface $M \subset \mathbb{R}^{3}$ with finite total curvature and conformal compactification $\bar{M}$, let $B(N) \subset \bar{M}$ be the set of branch points of the extended Gauss map and $\mathcal{M}(N)$ the linear space of all complete, branched minimal immersions (including the constant maps) $X: \bar{M}-B(N) \rightarrow \mathbb{R}^{3}$ with the same Gauss map $N$ as $M$.

Theorem 2.29 (Montiel, Ros [150). Let $M \subset \mathbb{R}^{3}$ be a complete, immersed minimal surface with finite total curvature 23 Then there exists a linear map $u \in \mathcal{K}(M) \mapsto$ $X_{u} \in \mathcal{M}(N)$ such that the support function 24 of $X_{u}$ is $u$, and $u \in \mathcal{L}(M)$ if and only if $X_{u}$ is constant. Furthermore, this linear map gives rise to an isomorphism between the quotient spaces $\mathcal{K}(M) / \mathcal{L}(M)$ and $\mathcal{M}(N) /\{$ constants $\}$.

Among the admissible conformal metrics which can be used to express questions related with the Jacobi operator, a particularly interesting choice comes from consideration of the pullback metric $d s_{N}^{2}$ through the Gauss map $N: M \rightarrow \mathbb{S}^{2}$ from the standard spherical metric on $\mathbb{S}^{2}$. The metric $d s_{N}^{2}$ has singularities at the branch points of $N$, and the Jacobi operator transforms into $L_{N}=\Delta_{N}+2$, where $\Delta_{N}$ is the Laplace operator of $d s_{N}^{2}$. Eigenvalues and eigenfunctions of $L_{N}$ are well defined by a variational approach (Tysk 203]). In particular, the index of stability of a subdomain $\Omega \subset M$ with compact closure is equal to the number of eigenvalues of $\Delta_{N}$ which are strictly less than 2 , and the nullity of $\Omega$ is the multiplicity of 2 as an eigenvalue of $\Delta_{N}$. Using these ideas, Montiel and Ros gave some estimates for the index and nullity under different geometrical assumptions, among which we emphasize the following one.

Theorem 2.30 (Montiel, Ros 150). Let $M \subset \mathbb{R}^{3}$ be a complete, immersed minimal surface with finite total curvature. 23 If all the branch values of the Gauss map of $M$ lie on an equator of $\mathbb{S}^{2}$, then the dimension of $\mathcal{K}(M)$ is 3 and $M$ has index $2 d-1$, where $d$ is the degree of the extended Gauss map.

2.9. Barrier constructions. Barrier constructions allow one to construct compact and non-compact stable minimal surfaces in $\mathbb{R}^{3}$ that are constrained to lie in subdomains of $\mathbb{R}^{3}$ whose boundaries have non-negative mean curvature. To illustrate this construction, we next give an example. Consider two disjoint, connected, properly embedded minimal surfaces $M_{1}, M_{2}$ in $\mathbb{R}^{3}$ and the closed connected region $W$ of $\mathbb{R}^{3}$ with $\partial W=M_{1} \cup M_{2}$. We now show how to produce compact, stable, embedded minimal surfaces in $W$. First note that $W$ is a complete flat three-manifold with boundary $\partial W$, and $\partial W$ has mean curvature zero. Meeks and Yau [145] proved that $W$ embeds isometrically in a homogeneously regular Riemannian three-manifold $(\widetilde{W}, \widetilde{g})$, with $\widetilde{W}$ diffeomorphic to the interior of $W$. Morrey 151 proved that in a homogeneously regular manifold, one can solve the classical Plateau problem and other area-minimizing problems. In particular, if $\Gamma$ is an embedded 1-cycle in $\widetilde{W}$ that bounds an orientable chain 25 in $\widetilde{W}$, then by standard results in geometric measure theory [56, $\Gamma$ is the boundary of a compact, least-area embedded surface $\Sigma_{\Gamma}(\widetilde{g}) \subset \widetilde{W}$. Meeks and Yau proved that the metric $\widetilde{g}$ on $\widetilde{W}$ can be approximated by a family of homogeneously regular metrics

\footnotetext{
${ }^{23}$ Theorems 2.29 and 2.30 remain valid for complete minimal surfaces in any quotient of $\mathbb{R}^{3}$ where the Gauss map makes sense, and which have finite total curvature in the quotient.

${ }^{24}$ The support function $u$ of an immersion $X: M \rightarrow \mathbb{R}^{3}$ is $u(p)=\langle p, N(p)\rangle$, where $N$ is the normal unit vector field of $X$; note that $u$ is the normal part of the variational field of $X$ by homotheties.

${ }^{25}$ We are considering here a finite sum of differentiable simplices.
} 
$\left\{g_{n}\right\}_{n \in \mathbb{N}}$ on $\widetilde{W}$, which converges smoothly on compact subsets of $\widetilde{W}$ to $\widetilde{g}$, and each $g_{n}$ satisfies a convexity condition outside of $W \subset \widetilde{W}$, which forces the least-area surface $\Sigma_{\Gamma}\left(g_{n}\right)$ to lie in $W$ if $\Gamma$ lies in $W$. A subsequence of the $\Sigma_{\Gamma}\left(g_{n}\right)$ converges to a smooth minimal surface $\Sigma_{\Gamma}$ of least area in $W$ with respect to the original flat metric, thereby finishing our description of the barrier construction.

We now use this barrier construction to prove the following theorem due to Hoffman and Meeks [82, from which Theorem 2.17 follows directly.

Theorem 2.31 (Strong Half-space Theorem [82]). If $M_{1}$ and $M_{2}$ are two disjoint, proper, possibly branched minimal surfaces in $\mathbb{R}^{3}$, then $M_{1}$ and $M_{2}$ are parallel planes.

Proof. Let $W$ be the closed complement of $M_{1} \cup M_{2}$ in $\mathbb{R}^{3}$ that has portions of both $M_{1}$ and $M_{2}$ on its boundary. As explained above, the surface $\partial W$ is a good barrier for solving Plateau-type problems in $W$. Let $M_{1}(1) \subset \cdots \subset M_{1}(n) \subset \cdots$ be a compact exhaustion of $M_{1}$ and let $\Sigma_{1}(n)$ be a least-area surface in $W$ with boundary $\partial M_{1}(n)$. Let $\alpha$ be a compact arc in $W$ which joins a point in $M_{1}(1)$ to a point in $\partial W \cap M_{2}$. By elementary intersection theory, $\alpha$ intersects every leastarea surface $\Sigma_{1}(n)$. By compactness of least-area surfaces, a subsequence of the surfaces $\Sigma_{1}(n)$ converges to a properly embedded area-minimizing surface $\Sigma$ in $W$ with a component $\Sigma_{0}$ which intersects $\alpha$; this proof of the existence of $\Sigma$ is due to Meeks, Simon and Yau 139. Since $\Sigma_{0}$ separates $\mathbb{R}^{3}, \Sigma_{0}$ is orientable and so by Theorem 2.25, $\Sigma_{0}$ is a plane. Hence, $M_{1}$ and $M_{2}$ lie in closed half-spaces of $\mathbb{R}^{3}$. Then, the height function of $M_{1}, M_{2}$ over their separating plane is a positive harmonic function, which must be constant provided that $M_{1}$ and $M_{2}$ are recurrent (see Definition 7.2 and Proposition 7.4). The fact that $M_{1}$ and $M_{2}$ are recurrent will be proved in Theorem 7.7 below. Hence, $M_{1}$ and $M_{2}$ must be planes 26

Another useful application of the barrier construction is the following. Suppose $\Gamma$ is an extremal simple closed curve in $\mathbb{R}^{3}$, i.e., that $\Gamma$ lies on the boundary of its convex hull $B$. We first assume that $\partial B$ is smooth. By the Jordan Curve Theorem, $\Gamma$ is the boundary of two disks $D_{1}, D_{2} \subset \partial B$. Suppose $\Gamma$ bounds two different compact, branched minimal immersions and let $\Sigma$ denote their union. By the convex hull property $27 \Sigma \subset B$. Let $W_{1}, W_{2}$ be the geodesic completion 28 of the two components of $B-\Sigma$ that contain the disks $D_{1}, D_{2}$. In this case, $\partial W_{1}$ and $\partial W_{2}$ consist of smooth pieces with non-negative mean curvature and convex corners. Meeks and Yau 145. proved that such boundaries are good barriers for solving least-area problems. In fact, in this case they proved that $\Gamma$ bounds a leastarea embedded disk $\widetilde{D}_{1} \subset W_{1}$ and a different least-area embedded disk $\widetilde{D}_{2} \subset W_{2}$. Similarly, if $\Gamma$ bounds a unique, branched minimal surface which is not an embedded stable minimal disk, then with this barrier argument, we can produce two different

\footnotetext{
${ }^{26}$ The original proof of the Strong Half-space Theorem uses the Half-space Theorem (Theorem 2.17] to conclude that $M_{1}, M_{2}$ are planes, once one knows that $\Sigma_{0}$ is a separating plane. Here we have used Proposition 7.4 since we wanted to deduce the Half-space Theorem 2.17 as a consequence of Theorem 2.31

${ }^{27}$ This property states that any compact minimal surface with boundary lies in the convex hull of its boundary. It works in much more generality than for minimal surfaces; in fact, Osserman 162 characterized the surfaces in $\mathbb{R}^{3}$ all of whose precompact subdomains lie in the convex hull of their boundaries, as those surfaces with non-positive Gaussian curvature.

${ }^{28}$ The geodesic completion of a Riemannian manifold is the completion of the underlying intrinsic metric space structure defined by the infimum of the lengths of curves in that manifold.
} 
embedded minimal disks with boundary $\Gamma$. If $\partial B$ is not assumed to be smooth, then one can use an approximation argument by convex smooth boundaries to obtain the same conclusion (see, e.g., [144).

On the other hand, Nitsche [159 proved that a regular, analytic Jordan curve in $\mathbb{R}^{3}$ whose total curvature is at most $4 \pi$ bounds a unique minimal disk. The hypothesis of analyticity for the boundary curve in Nitsche's Theorem comes from consideration of boundary branch points. When $\Gamma$ is of class $C^{2}$ and extremal, there are never boundary branch points, as shown in 145]; one uses here Hildebrandt's boundary regularity results [70. The last two paragraphs can be summarized in the following statement.

Theorem 2.32 (Meeks, Yau [145]). If $\Gamma \subset \mathbb{R}^{3}$ is a $C^{2}$-extremal curve with total curvature at most $4 \pi$, then $\Gamma$ is the boundary of a unique, compact, branched minimal surface and this surface is an embedded minimal disk of least area.

\section{Minimal surfaces With finite topology AND More than one End}

Collin's Theorem 1.3 stated in the introduction is a key result which reduces the study of properly embedded minimal surfaces in $\mathbb{R}^{3}$ with finite topology and at least two ends (see Definition 2.20 for the general definition of end) to the family of surfaces with finite total curvature. As mentioned in Section 2.3. the powerful theory of compact Riemann surfaces applies to these last surfaces, which has helped to make possible a rather good understanding of them. In this section we have two goals: to mention the main construction methods that are used to produce complete, embedded minimal surfaces of finite total curvature, and to state uniqueness and non-existence results for these kinds of surfaces. We will start with this last goal.

3.1. Classification results for embedded minimal surfaces of finite total curvature. The Jorge-Meeks formula (equation (91)) relates the total curvature, genus and number of ends of a complete, embedded minimal surface with finite total curvature. Consequently, it is natural to look for classification and nonexistence results fixing two of these numbers, or even better, fixing only one of the three. The first deep result along these lines is due to Schoen, who proved the following theorem in 1983 as an application of the Alexandrov reflection technique (see Alexandrov [2] and Hopf [86 for a description of this classical technique).

Theorem 3.1 (Schoen 188). The catenoid is the unique complete, immersed minimal surface in $\mathbb{R}^{3}$ with finite total curvature and two embedded ends.

Eight years later, López and Ros classified the embedded genus-zero examples of finite total curvature. The main ingredients in the proof of the result below are the López-Ros deformation explained in Section 2.2 together with the maximum principle for minimal surfaces.

Theorem 3.2 (López, Ros [104]). The plane and the catenoid are the only complete, embedded minimal surfaces in $\mathbb{R}^{3}$ with genus zero and finite total curvature.

Costa 43, 44, showed that any complete, embedded minimal surface in $\mathbb{R}^{3}$ with genus one and three ends either is the Costa surface or lies in the Hoffman-Meeks deformation discussed in Section 2.5. with the moduli space of complete, embedded, minimal, thrice-punctured tori being diffeomorphic to an open interval. This 
was the first time that a space of complete, embedded minimal surfaces with a prescribed topology had an explicit description as a manifold of finite positive dimension. Concerning moduli spaces of minimal surfaces with finite total curvature and prescribed topology, Pérez and Ros [170, gave general conditions on the space $\mathcal{M}(g, r)$ whose elements are the complete, embedded minimal surfaces in $\mathbb{R}^{3}$ with finite total curvature, genus $g$ and $r$ ends, to have a structure of a real analytic manifold of dimension $r-2$ around a given minimal surface $M \in \mathcal{M}(g, r)$, and they called such conditions on $M$ the non-degeneracy of the surface. This term comes from the fact that the manifold structure on $\mathcal{M}(g, r)$ follows from an application of the Implicit Function Theorem, which needs a certain derivative to be surjective (also sometimes called non-degenerate). The non-degeneracy condition is expressed in terms of the bounded Jacobi functions on $M$ (see Section 2.8 for the definition of Jacobi function). They also identified the tangent space to $\mathcal{M}(g, r)$ at a non-degenerate minimal surface $M$ with the set of Jacobi functions on $M$ which have at most logarithmic singularities at the ends. Other compactness results for moduli spaces of complete, embedded minimal surfaces with finite total curvature have been given by Ros [177], Traizet [198] and by Meeks, Pérez and Ros [119, 129]. We will explain in Section 10 some further advances in this area; see specifically Theorem 1.6 stated in the Introduction.

3.2. Methods for constructing properly embedded minimal surfaces of finite total curvature. The Weierstrass representation gives a direct method for constructing minimal surfaces of finite total curvature, starting from an input which is a compact Riemann surface $\bar{M}$ and three meromorphic 1-forms $\phi_{1}, \phi_{2}, \phi_{3}$ on $\bar{M}$ satisfying $\sum_{i} \phi_{i}^{2}=0$. One then defines the (stereographically projected) Gauss map as $g=\frac{\phi_{3}}{\phi_{1}-i \phi_{2}}$, and the height differential as $d h=\phi_{3}$. The degrees of freedom to work with come from the moduli space of conformal structures for a given genus, together with the points which correspond to the ends (poles of $\phi_{i}$, $i=1,2,3$ ). Avoiding branch points for the induced metric (condition $i$ ) in Theorem 2.10) amounts to a rather simple problem of adjusting the canonical divisors of the 1-forms $\phi_{1}, \phi_{2}, \phi_{3}$. The first main task is to solve the period problem (condition ii) in Theorem 2.10). Since the extrinsic symmetries of a minimal surface simplify the period calculations with the Weierstrass data, the period problem is sometimes tackled by assuming enough symmetries; in this way, the constraint equations in (5) reduce to a sufficiently small number of closed curves in $M=\bar{M}-\{$ ends $\}$, so that one can adjust the remaining free parameters, often by continuity or degree-type arguments, and solve the period problem. A variant of this idea is to express the period problem as a Teichmüller theory issue (we will explain more about this below; see the method of Weber and Wolf). An even more serious difficulty appears when we try to obtain an embedded example, since the Weierstrass data gives no direct information on this matter. A useful technique for proving embeddedness is to decompose $M$ into congruent pieces (which are fundamental domains of the action of the symmetry group of $M$, so we need again to assume enough symmetries here), and to prove that each piece is embedded.

Another remarkable method to produce examples is to perturb singular configurations (i.e., minimal surfaces with singularities, modeled on Riemann surfaces with nodes) via the Implicit Function Theorem, which is the main idea of the method of Traizet, or to desingularize configurations of smooth minimal surfaces, first by constructing a surface of mean curvature approximately zero, and then by applying 
a fixed point theorem to obtain a nearby minimal surface (method of Kapouleas). Next we will explain a bit more about these three different methods. If we were to follow the historical order, then Kapouleas's method would be first; nevertheless, we will leave it to the end of this section since the other two methods rely on the Weierstrass representation (which we started this section with), while Kapouleas's method uses PDE and functional analysis arguments.

The method of Weber and Wolf. The goal is to produce a surface with prescribed topology and symmetries via the Weierstrass representation, although this method has interesting features that differ from the general presentation given above. The key idea is an original viewpoint to solve the period problem, based on flat structures. If $\alpha$ is a meromorphic 1 -form on a Riemann surface $\mathcal{R}$, then $|\alpha|^{2}$ defines a flat metric on $\mathcal{R}-\alpha^{-1}(\{0, \infty\})$. In particular, we can develop $\mathcal{R}$ locally as a domain $\Omega_{\alpha}$ in $\mathbb{C}$ (so that the usual holomorphic differential $d z$ pulls back to $\alpha$ ), except around a zero or pole of $\alpha$, where the developing map produces a cone point (i.e., a generalized sector with edges identified, whose aperture angle is neither necessarily less than $2 \pi$ nor a rational multiple of $\pi$ ), whose cone angle depends explicitly on the order of the zero or pole of $\alpha$. The line element $|\alpha|$ associated to the metric $|\alpha|^{2}$ is called a flat structure on $\mathcal{R}$.

Once we understand the concept of flat structure, the method is easy to follow. One starts by assuming that the desired minimal surface $M$ exists and computes the divisors of the 1-forms $g d h$ and $g^{-1} d h$ (as usual, $g$ is the Gauss map and $d h$ the height differential of $M$; this divisor information can be read from the expected shape of the surface). Assuming enough symmetry, one supposes that a fundamental piece of the action of the symmetry group of $M$ is a disk $D$ with boundary and develops $D$ as two domains $\Omega_{g d h}, \Omega_{g^{-1} d h}$ with piecewise smooth boundary in $\mathbb{C}$ as we explained above for $\alpha$. The periods of $g d h$ and $g^{-1} d h$ transform into integrals of $d z$ along paths in $\mathbb{C}$, and the complex period condition in equation (5)-left is equivalent to the fact that the developing domains in $\mathbb{C}$ associated to $g d h$ and $g^{-1} d h$ are conjugate in a natural sense. This period condition is, thus, automatically satisfied by taking appropriate pairs of developing domains. The real period condition in equation (5)-right is also easy to fulfill, since it reduces to an algebraic condition on the sum of the cone angles. The reader could think that the period problem has been magically solved, but in reality we have only exchanged it with another problem in Teichmüller theory: find a pair of conjugate developing domains $\Omega_{g d h}, \Omega_{g^{-1} d h}$ so that they match up to give a well-defined Riemann surface on which $g d h, g^{-1} d h$ both exist (once this difficulty is solved, the Weierstrass data $(g, d h)$ is easily recovered from $\left.g d h, g^{-1} d h\right)$. This task is done by studying the moduli space of pairs of conjugate domains and using specific tools of Teichmüller theory, such as extremal length.

Weber and Wolf have used this method in several different situations 204, 205. For instance, they produced for all odd genera $g$, a complete minimal surface $M(g) \subset \mathbb{R}^{3}$ with finite total curvature, genus $g$, two catenoidal ends and $g$ planar horizontal ends. Furthermore, $M(g)$ is embedded outside a compact set 29 and the symmetry group of $M(g)$ is generated by reflective symmetries about a pair of orthogonal vertical planes and a rotational symmetry about a horizontal line. The surface $M(g)$ also has theoretical importance, since if it were proved to be

\footnotetext{
${ }^{29}$ The conjecture that the surfaces $M(g)$ are all embedded is supported by graphical and numerical evidence.
} 
embedded, then it would represent the borderline case for Conjecture 11.5 below. Also, the surfaces $M(g)$ might be used to produce as a limit as $g \rightarrow \infty$, a properly embedded minimal surface in $\mathbb{R}^{3}$ with exactly one limit end and infinite genus.

The method of Traizet. Again this method is based on the Weierstrass representation, but with a different flavor from the preceding one. The technique also applies to construct minimal surfaces with infinite total curvature, but we will focus here on the finite total curvature case. The key point is that certain continuous families of embedded minimal surfaces $M(\theta)$ (here $\theta$ is a multi-parameter) with the same topology and total curvature have a weak limit as $\theta$ approaches a point $\theta_{\infty}$ in the boundary of the range of allowed parameters. A weak limit is a finite collection of minimal surfaces $\left\{M_{1, \infty}, \ldots, M_{k, \infty}\right\}$ such that:

- For each $i, M_{i, \infty}$ is a smooth limit of suitable rescalings of the $M(\theta)$ as $\theta \rightarrow \theta_{\infty}$.

- The topology and total curvature of the $M(\theta)$ can be recovered as a sum of the ones of all the $M_{i, \infty}, i=1, \ldots, k$.

This phenomenon allows one to model the weak limit as being a compact Riemann surface with nodes 30 Now the method allows one to go backwards: starting from a finite configuration of minimal surfaces $M_{1, \infty}, \ldots, M_{k, \infty}$ with finite total curvature modeled over a compact Riemann surface with nodes $\mathcal{R}(0)$, one studies how to deform $\mathcal{R}(0)$ by opening slightly its nodes, creating in this way an analytic family $\mathcal{R}(t)$ of compact Riemann surfaces without nodes, where $t$ is a vector-valued complex parameter around $t=0$. At the same time, the collection of Weierstrass representations $\left(g_{i, \infty}, d h_{i, \infty}\right)$ of the $M_{i, \infty}$ on $\mathcal{R}(0)$ deform analytically to a global Weierstrass representation $\left(g_{t}, d h_{t}\right)$ on $\mathcal{R}(t)$, and we want to find some subfamily of parameters $t$ such that the period problem for the triple $\left(\mathcal{R}(t), g_{t}, d h_{t}\right)$ is solved. To solve this period problem, one writes its solutions as the zeros of an analytic map $P$ from the $t$-domain into certain $\mathbb{C}^{m}$. Furthermore, $P(0)=0$ since the $M_{1, \infty}, \ldots, M_{k, \infty}$ are real minimal surfaces. The desired family of zeros of $P$ passing through $t=0$ is then obtained by application of the Implicit Function Theorem, provided that a certain non-degeneracy condition holds for the limit configuration (namely, the differential of $P$ at $t=0$ must be surjective).

It is remarkable that Traizet's method makes no restrictions a priori either on the genus of the surfaces to be constructed, or on their symmetry groups. In fact, Traizet applied this method to produce the first example of a complete, embedded minimal surface with finite total curvature in $\mathbb{R}^{3}$ and trivial symmetry group; see [197. For this result, Traizet desingularized a configuration of planes and tiny catenoids. One limitation of the method is that, by its nature, it only produces examples which are close to the boundary of their corresponding moduli spaces. As said before, the method also works for surfaces of infinite total curvature. For instance, Traizet and Weber 201 have used these ideas to perturb weak limits of planes and helicoids, thereby producing the first non-classical examples of minimal parking garage surfaces (see the comments after Theorems 4.5 and 4.7 also see the survey [116 for a more detailed explanation of this concept). Very recently, Traizet 200 has applied this method to produce a properly embedded minimal surface in $\mathbb{R}^{3}$ with infinite genus and one limit end; see Footnote 43 for the importance of this new example.

\footnotetext{
${ }^{30}$ See [88 page 245] for the definition of a Riemann surface with nodes.
} 
We also remark that according to Traizet 197, his technique was inspired on a clever modification of an argument due to Meeks, Pérez and Ros [125, used in proving the uniqueness of the Riemann minimal examples in their natural periodic moduli space, around a weak limit given by infinitely many catenoids and planes.

The method of Kapouleas. This method differs in an essential manner from the preceding ones, since it is not based on the Weierstrass representation but on PDE theory and functional analysis techniques. One starts by considering a finite collection $\mathcal{F}$ of horizontal planes and catenoids with axis the $x_{3}$-axis. The goal is to desingularize $\mathcal{F}$ to obtain a complete minimal surface $M \subset \mathbb{R}^{3}$ of finite total curvature. By desingularization, we mean here that each circle $C$ of intersection of the planes and/or catenoids in $\mathcal{F}$ is replaced in a first stage by a suitably bent, slightly deformed and appropriately scaled down singly periodic Scherk minimal surface $\mathcal{S}_{\theta}$, in the same way that $\mathcal{S}_{\theta}$ can be viewed as the desingularization of a pair of planes intersecting at angle $\theta$ (see Figure 3, center for an indication of the geometry of the replaced neighborhood near $C$ when $\theta=\pi / 2$ ). The fact that one desingularizes a circle instead of a straight line can be overcome by thinking of a line as the limit of a circle with arbitrarily large radius, or equivalently, by scaling down and slightly bending the Scherk connection piece. The removing of all the intersection circles decomposes the catenoids and planes in $\mathcal{F}$ into bounded pieces (namely discs and annuli) and annular ends, all of which, after being slightly perturbed, glue to the wings of the bent Scherk pieces. In this way, one constructs a smooth (non-minimal) surface $M_{1}$, which is the base of the second stage of the method: perturb $M_{1}$ by small normal graphs, to find a minimal surface $M$ which is the final goal of the method. Since the surface $M_{1}$ has a large number of handles (depending on the precision of the desingularization in the first stage), one does not have a control on a lower bound for the genus of the final minimal surface $M$. The annular ends of the original configuration $\mathcal{F}$, viewed as graphs of logarithmic growth over the $\left(x_{1}, x_{2}\right)$-plane, change after the perturbation in the second stage, to give ends of the minimal surface $M$ with slightly different logarithmic growths and positions from the original ones. In particular, planar ends may become asymptotic to catenoids of small logarithmic growths. But this change is not explicit, since the second stage is solved by an application of a fixed point theorem in a suitable space of functions. In order to insure embeddedness of $M$, one must then start from a configuration $\mathcal{F}$ having all its ends with different logarithmic growths (in particular, $\mathcal{F}$ contains at most one plane). For details, see Kapouleas [90.

A good example for understanding the essence of this method is to consider the Costa-Hoffman-Meeks surfaces $M_{k, 1}$ described in Section 2.5. Hoffman and Meeks [80. proved that after suitable normalizations, the $M_{k, 1}$ converge as $k \rightarrow \infty$ to the following two limits:

- The union of a catenoid and a plane passing through its waist circle (this limit is attained by fixing the logarithmic growth of the catenoidal ends of $\left.M_{k, 1}\right)$.

- The singly periodic Scherk minimal surface $\mathcal{S}_{\theta=\pi / 2}$ (after normalizing the maximal absolute curvature of $M_{k, 1}$ to be some fixed positive number).

This phenomenon for the surfaces $M_{k, 1}$ with $k$ large motivated the question of whether it is possible to desingularize the intersection set $I$ of two transversely intersecting minimal surfaces, by replacing neighborhoods of $I$ with necklaces of 
singly periodic Scherk minimal surfaces 31 Kapouleas used his method in 90 to perform this desingularization under the assumption of coaxial symmetry. In [91, he announced a general theorem for intersecting minimal surfaces in Riemannian three-manifolds, without symmetry assumptions, based on the same method.

\section{Sequences of Embedded minimal Surfaces WITHOUT UNIFORM LOCAL AREA BOUNDS}

Two central problems in minimal surface theory are to understand the possible geometries or shapes of embedded minimal surfaces in $\mathbb{R}^{3}$ of finite genus, as well as the structure of limits of sequences of embedded minimal surfaces with fixed genus. The classical theory deals with these limits when the sequence has uniform local area and curvature bounds, since in this case one can reduce the problem of taking limits of minimal surfaces to taking limits of solutions of the minimal surface equation (for this reduction, one uses the local curvature bound in order to express the surfaces as local graphs of uniform size, and the local area bound to constrain locally the number of such graphs to a fixed finite number), which follows from the classical Arzelà-Ascoli theorem; see, e.g., 171]. Hence, we will concentrate here on the case where we do not have such estimates. The understanding of these problems starts with the analysis of the local structure in a fixed extrinsic ball, an issue tackled by Colding and Minicozzi in a series of papers where they study the structure of a sequence of compact, embedded minimal surfaces $M_{n}$ with fixed genus and no area bounds in balls $\mathbb{B}_{n} \subset \mathbb{R}^{3}$, whose radii tend to infinity as $n \rightarrow \infty$ and with boundaries $\partial M_{n} \subset \partial \mathbb{B}_{n}, n \in \mathbb{N}$.

4.1. Colding-Minicozzi theory for locally simply connected sequences of minimal surfaces. As we said above, a main goal of the Colding-Minicozzi theory is to understand the limit objects for a sequence of compact, embedded minimal surfaces $M_{n}$ with fixed genus but not a priori area bounds, each one with boundary contained in the boundary sphere of a Euclidean ball centered at the origin, say with radius $R_{n}>0$. Typically, one finds minimal lamination 32 as limits of subsequences of the $M_{n}$. Nevertheless, we will see in Theorems 4.2 and 4.7 versus Example II of Section 4.2, that the behavior of the limit lamination changes dramatically depending on whether $R_{n}$ diverges to $\infty$ or stays bounded, in the sense that the limit lamination can develop removable or essential singularities. This phenomenon connects with another main problem in the current state of the theory of minimal surfaces: finding removable singularity theorems for minimal laminations, or equivalently, finding extension theorems for minimal laminations defined outside of a small set. The interested reader can find details about removable singularity results in Meeks, Pérez and Ros 122; also see Conjectures 10.4 and 11.3 below.

Coming back to the Colding-Minicozzi results, the most important structure theorem in this theory is when the $M_{n}$ are disks whose Gaussian curvature blows up near the origin. The basic example in this setting is a sequence of rescaled helicoids $M_{n}=\lambda_{n} H=\left\{\lambda_{n} x \mid x \in H\right\}$, where $H$ is a fixed vertical helicoid with axis the $x_{3}$-axis and $\lambda_{n} \in \mathbb{R}^{+}, \lambda_{n} \searrow 0$. The curvature of the sequence $\left\{M_{n}\right\}_{n}$ blows up along the $x_{3}$-axis and the $M_{n}$ converge away from the axis to the foliation $\mathcal{L}$ of

${ }^{31} \mathrm{~A}$ related interesting question is if one could use a model different from a singly periodic Scherk minimal surface, to desingularize two intersecting embedded minimal surfaces. This open problem is closely related to Conjecture 11.12 below.

${ }^{32}$ See Definition 4.4 for the concept of minimal lamination. 
$\mathbb{R}^{3}$ by horizontal planes. The $x_{3}$-axis is the singular set of $C^{1}$-convergence $S(\mathcal{L})$ of $M_{n}$ to $\mathcal{L}$ (i.e., the $M_{n}$ do not converge $C^{1}$ to the leaves of $\mathcal{L}$ along the $x_{3}$-axis), but each leaf $L$ of $\mathcal{L}$ extends smoothly across $L \cap S(\mathcal{L})$ (i.e., $S(\mathcal{L})$ consists of removable singularities of $\mathcal{L}$ ).

Definition 4.1. In polar coordinates $(\rho, \theta)$ on $\mathbb{R}^{2}-\{0\}$ with $\rho>0$ and $\theta \in \mathbb{R}$, a $k$-valued graph on an annulus of inner radius $r$ and outer radius $R$, is a singlevalued graph of a function $u(\rho, \theta)$ defined over $\{(\rho, \theta)|r \leq \rho \leq R,| \theta \mid \leq k \pi\}, k$ being a positive integer. The separation between consecutive sheets is $w(\rho, \theta)=$ $u(\rho, \theta+2 \pi)-u(\rho, \theta) \in \mathbb{R}$.

With this notation at hand, the statement of the so-called Limit Lamination Theorem for Disks (Theorem 0.1 of [34) can be easily understood. Given $p \in \mathbb{R}^{3}$ and $R>0$, we denote by $\mathbb{B}(p, R)=\left\{x \in \mathbb{R}^{3} \mid\|x-p\|<R\right\}, \mathbb{B}(R)=\mathbb{B}(\overrightarrow{0}, R)$ and $K_{M}$ the Gaussian curvature function of a surface $M$.

Theorem 4.2 (Limit Lamination Theorem for Disks, Colding and Minicozzi 34). Let $M_{n} \subset \mathbb{B}\left(R_{n}\right)$ be a sequence of embedded minimal disks with $\partial M_{n} \subset \partial \mathbb{B}\left(R_{n}\right)$ and $R_{n} \rightarrow \infty$. If $\sup \left|K_{M_{n} \cap \mathbb{B}(1)}\right| \rightarrow \infty$, then there exists a subsequence of the $M_{n}$ (denoted in the same way) and a Lipschitz curve $S: \mathbb{R} \rightarrow \mathbb{R}^{3}$ such that up to a rotation of $\mathbb{R}^{3}$ :

1. $x_{3}(S(t))=t$ for all $t \in \mathbb{R}$.

2. Each $M_{n}$ consists of exactly two multigraphs away from $S(\mathbb{R})$ (which spiral together).

3. For each $\alpha \in(0,1)$, the surfaces $M_{n}-S(\mathbb{R})$ converge in the $C^{\alpha}$-topology to the foliation $\mathcal{L}=\left\{x_{3}=t\right\}_{t \in \mathbb{R}}$ by horizontal planes.

4. $\sup \left|K_{M_{n} \cap \mathbb{B}(S(t), r)}\right| \rightarrow \infty$ as $n \rightarrow \infty$, for any $t \in \mathbb{R}$ and $r>0$.

Theorem 4.2 has two main ingredients in its proof, which we explain very roughly. The first ingredient is that the embedded minimal disk $M_{n}$ with large curvature at some interior point can be divided into building blocks, each one being a multivalued graph $u_{n}(\rho, \theta)$ defined on an annulus, and that these basic pieces fit together properly. In particular, Colding and Minicozzi proved that the number of sheets of $u_{n}(\rho, \theta)$ rapidly grows as the curvature blows up as $n$ goes to infinity and at the same time, the sheets of $u_{n}$ do not accumulate in a half-space. This is obtained by means of sublinear and logarithmic bounds for the separation $w_{n}(\rho, \theta)$ (see Definition 4.1) as a function of $\rho \rightarrow \infty$; by these bounds we mean the following inequalities; see Corollary 11.2 in Colding and Minicozzi [30 and Theorem 0.8 in [27:

$$
\left|w_{n}\right|\left(\rho_{2}, 0\right) \leq\left|w_{n}\right|\left(\rho_{1}, 0\right)\left(\frac{\rho_{2}}{\rho_{1}}\right)^{\beta}, \quad \frac{1}{C \log \rho} \leq \frac{w_{n}(\rho, 0)}{w_{n}(1,0)} \leq C \log \rho,
$$

where $\beta \in(0,1), C>0$ are constants independent of $n$.

Another consequence of these bounds is that by allowing the inner radius $r$ of the annulus where the multigraph is defined to go to zero, the sheets of the multigraphs $u_{n}$ collapse (i.e., $\left|w_{n}(\rho, \theta)\right| \rightarrow 0$ as $n \rightarrow \infty$ for $\rho, \theta$ fixed); thus a subsequence of the $u_{n}$ converges to a smooth minimal graph through $r=0$. The fact that the $R_{n}$ go to $\infty$ then implies that this limit graph is entire and, by Bernstein's Theorem [6], it is a plane. 


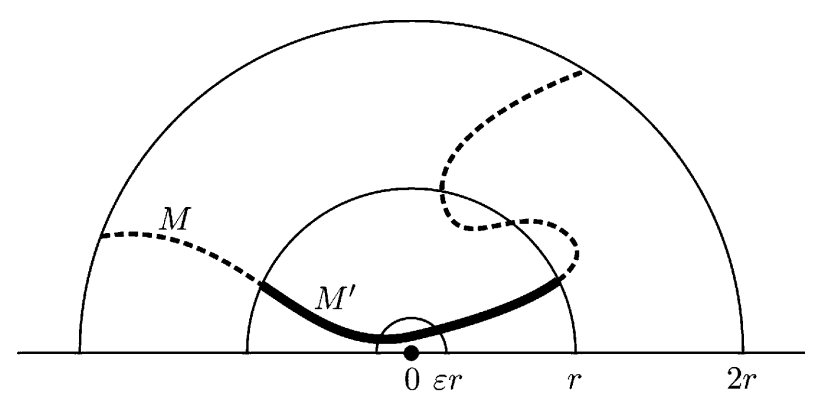

FiguRE 10. The one-sided curvature estimate.

The second main ingredient in the proof of Theorem 4.2 is the so-called onesided curvature estimate, a scale-invariant bound for the Gaussian curvature of any embedded minimal disk in a half-space.

Theorem 4.3 (Colding and Minicozzi 34). There exists an $\varepsilon>0$ such that the following holds. Given $r>0$ and an embedded minimal disk $M \subset \mathbb{B}(2 r) \cap\left\{x_{3}>0\right\}$ with $\partial M \subset \partial \mathbb{B}(2 r)$, then for any component $M^{\prime}$ of $M \cap \mathbb{B}(r)$ which intersects $\mathbb{B}(\varepsilon r)$,

(See Figure 10.)

$$
\sup _{M^{\prime}}\left|K_{M}\right| \leq r^{-2} \text {. }
$$

The hypothesis on $M$ to be simply connected in Theorem 4.3 is necessary, as the catenoid demonstrates (see Figure 13 left). Actually the 1-sided curvature estimate is equivalent to the property that every component of $M \cap \mathbb{B}(r)$ which intersects $\mathbb{B}(\varepsilon r)$ is a graph of small gradient over its projection on the $\left(x_{1}, x_{2}\right)$ plane. This result is needed in the proof of Theorem 4.2 in the following manner: once it has been proven that an embedded minimal disk $M$ contains a highly sheeted double multigraph $\widetilde{M}$, then $\widetilde{M}$ plays the role of the plane in the one-sided curvature estimate, which implies that reasonably large pieces of $M$ consist of multigraphs away from a cone with axis "orthogonal" to the double multigraph. The proofs of Theorems 4.2 and 4.3 are long and delicate. References [28, 29, 30, 35, 36] by Colding and Minicozzi are reading guides for the complete proofs of these results, which go through various papers [31, 32, 34].

Theorems 4.2 and 4.3 have been applied to obtain a number of results, among which we highlight three: Meeks and Rosenberg [136] proved that the helicoid and the plane are the unique properly embedded, simply connected minimal surfaces in $\mathbb{R}^{3}$ (see Theorem 1.4); Meeks, Pérez and Ros used Colding-Minicozzi theory to study properly embedded minimal surfaces in $\mathbb{R}^{3}$ with finite genus and infinitely many ends, proving that such a surface $M$ has necessarily two limit ends (Theorem 10.3 below); finally, the same authors proved (Theorem 1 in [126]) that if the genus of $M$ is zero, then its absolute Gaussian curvature function is bounded. These are key properties in the classification of the properly embedded, minimal planar domains in $\mathbb{R}^{3}$ (Theorem 1.2). We will discuss other applications of the Colding-Minicozzi results, such as Theorem 1.6.

We have mentioned the importance of understanding limits of sequences of embedded minimal surfaces with fixed (or bounded) genus but no a priori area bounds in a three-manifold $N$, a situation of which Theorem 4.2 is a particular case. This 
result shows that one must consider limit objects other than minimal surfaces, such as minimal foliations or, more generally, minimal laminations of $N$. We next define these objects in the case that $N$ is an open subset of $\mathbb{R}^{3}$, although the reader can easily extend the following definition to other ambient spaces.

Definition 4.4. A lamination of an open subset $U \subset \mathbb{R}^{3}$ is the union of a collection of pairwise disjoint, connected, injectively immersed surfaces, with a certain local product structure. More precisely, it is a pair $(\mathcal{L}, \mathcal{A})$ satisfying:

1. $\mathcal{L}$ is a closed subset of $U$.

2. $\mathcal{A}=\left\{\varphi_{\beta}: \mathbb{D} \times(0,1) \rightarrow U_{\beta}\right\}_{\beta}$ is a collection of coordinate charts of $\mathbb{R}^{3}$ (here $\mathbb{D}$ is the open unit disk, $(0,1)$ the open unit interval and $U_{\beta}$ an open subset of $U$ ).

3. For each $\beta$, there exists a closed subset $C_{\beta}$ of $(0,1)$ such that $\varphi_{\beta}^{-1}\left(U_{\beta} \cap \mathcal{L}\right)=$ $\mathbb{D} \times C_{\beta}$.

We will simply denote laminations by $\mathcal{L}$, omitting the charts $\varphi_{\beta}$ in $\mathcal{A}$. A lamination $\mathcal{L}$ is said to be a foliation of $U$ if $\mathcal{L}=U$. Every lamination $\mathcal{L}$ naturally decomposes into a union of disjoint connected surfaces, called the leaves of $\mathcal{L}$. As usual, the regularity of $\mathcal{L}$ requires the corresponding regularity on the change of coordinate charts. A lamination is minimal if all its leaves are minimal surfaces.

Each leaf of a minimal lamination $\mathcal{L}$ is smooth, and if $C$ is a compact subset of a limit lea $23 \in \mathcal{L}$, then the leaves of $\mathcal{L}$ converge smoothly to $L$ over $C$ (i.e., they converge uniformly in the $C^{k}$-topology on $C$ for any $k$ ). In general, a codimension-one minimal lamination of a Riemannian manifold is of class $C^{0,1}$ (see Proposition B.1 in Colding and Minicozzi [34 for the proof of this result when the leaves are two-dimensional). In particular, a codimension-one minimal foliation of a Riemannian manifold is of class $C^{0,1}$ and furthermore, the unit normal vector field is $C^{0,1}$ as well (see Solomon [191]).

The following result concerns the behavior of limit leaves for a minimal lamination, which generalizes some statements in Lemma A.1 in Meeks and Rosenberg [137.

Theorem 4.5 (Meeks, Pérez, Ros [130]). Any limit leaf L of a codimension-one minimal lamination of a Riemannian manifold is stable for the Jacobi operator 34 More strongly, every two-sided cover of such a limit leaf $L$ is stable. Therefore, the collection of stable leaves of a minimal lamination $\mathcal{L}$ has the structure of a sublamination containing all the limit leaves of $\mathcal{L}$.

We next return to our discussion about Theorem 4.2. Using the regularity Theorem 9.6 below, one can replace the Lipschitz curve in Theorem 4.2 by a vertical line, which on large balls, yields what we refer to as a limiting parking garage structure on $\mathbb{R}^{3}$ with one column. We will find again a limiting parking garage structure in Theorem 4.7 below, but with two columns instead of one. In a parking garage structure one can travel quickly up and down the horizontal levels of the limiting

\footnotetext{
${ }^{33} \mathrm{~A}$ point $p \in \mathcal{L}$ is said to be a limit point if for some $\beta, \varphi_{\beta}^{-1}(p)=(x, y) \in \mathbb{D} \times C_{\beta}$, with $y$ being a limit point of $C_{\beta}$ (this definition does not depend on the local chart). A leaf $L$ of $\mathcal{L}$ is called a limit leaf if it contains a limit point, which is equivalent to consisting entirely of limit points.

34 The Jacobi operator of a two-sided minimal surface $M$ in a Riemannian three-manifold $N$ is $L=\Delta+\left(\operatorname{Ric}(\nu)+|\sigma|^{2}\right)$, where we are using the same notation as in Footnote 21]
} 
surfaces only along the (helicoidal) columns, in much the same way that some parking garages are configured for traffic flow, hence, the name parking garage structure. We refer the reader to [116] for further study of limiting parking garage structures.

Theorem 4.2 does not hold if we exchange the hypothesis that "the radii $R_{n}$ of the balls go to $\infty$ " by " $R_{n}$ equals a constant", as demonstrated by a counterexample due to Colding and Minicozzi 29]; see also Example II in Section 4.2 below. They constructed a sequence of embedded minimal disks $M_{n} \subset \mathbb{B}(1)$ with $\partial M_{n} \subset \partial \mathbb{B}(1)$, all passing through the origin $\overrightarrow{0}$, and with Gaussian curvature blowing up only at $\overrightarrow{0}$. This sequence produces a limit lamination of $\mathbb{B}(1)-\{\overrightarrow{0}\}$ with an isolated singularity at the origin. The limit lamination consists of three leaves, one of them being the flat horizontal punctured disk (which extends through $\overrightarrow{0}$ ), and the other two being non-proper multigraphs with this flat disk as limit set (see Figure 13, right for a similar example). In particular, both smoothness and properness of the leaves of the limit lamination fail for this local example. We refer the reader to the survey [116], where we will consider the general question of extending a minimal lamination $\mathcal{L}$ of $\mathbb{R}^{3}-A$, for certain closed subsets $A$ of $\mathbb{R}^{3}$, and also to Conjectures 10.4 and 11.3 below.

Theorem 4.2 deals with limits of sequences of disks, but it is also useful when studying more general situations, as for instance, locally simply connected sequences of minimal surfaces, a notion which we now define.

Definition 4.6. Suppose that $\left\{M_{n}\right\}_{n}$ is a sequence of embedded minimal surfaces (possibly with boundary) in an open set $U$ of $\mathbb{R}^{3}$. If for any $p \in U$ there exists a number $r(p)>0$ such that $\mathbb{B}(p, r(p)) \subset U$ and for $n$ sufficiently large, $M_{n}$ intersects $\mathbb{B}(p, r(p))$ in compact disks whose boundaries lie on $\partial \mathbb{B}(p, r(p))$, then we say that $\left\{M_{n}\right\}_{n}$ is locally simply connected in $U$. If $\left\{M_{n}\right\}_{n}$ is a locally simply connected sequence in $U=\mathbb{R}^{3}$ and the positive number $r(p)$ can be chosen independently of $p \in \mathbb{R}^{3}$, then we say that $\left\{M_{n}\right\}_{n}$ is uniformly locally simply connected.

There is a subtle difference between our definition of uniformly locally simply connected and that of Colding and Minicozzi [24, which may lead to some confusion. Colding and Minicozzi define a sequence $\left\{M_{n}\right\}_{n}$ to be uniformly locally simply connected in an open set $U \subset \mathbb{R}^{3}$ if for any compact set $K \subset U$, the number $r(p)$ in Definition 4.6 can be chosen independently of $p \in K$. It is not difficult to check that this concept coincides with our definition of a locally simply connected sequence in $U$.

The Limit Lamination Theorem for Disks (Theorem 4.2) admits a generalization to a locally simply connected sequence of non-simply connected planar domains, which we now explain since it will be useful for our goal of classifying minimal planar domains. Instead of the scaled-down limit of the helicoid, the basic example in this case is an appropriate scaled-down limit of Riemann minimal examples $M_{\lambda}, \lambda>0$, defined in Section 2.5. To understand this limit, one considers the flux $F(\lambda)$ of $M_{\lambda}$, which is the flux vector along any compact horizontal section, $M_{\lambda} \cap\left\{x_{3}=\right.$ constant $\}$, normalized so that the third component of this vector equals one. The fact that $M_{\lambda}$ is invariant by reflection in the $\left(x_{1}, x_{3}\right)$-plane $\Pi$ forces $F(\lambda)$ to be contained in $\Pi$ for each $\lambda$. Furthermore, $\lambda \mapsto F=F(\lambda)$ is a bijection that parameterizes the whole family of Riemann minimal examples, with $F$ running from horizontal to vertical (with monotonically increasing slope function). When $F$ tends to vertical, then it can be proved that $M_{\lambda(F)}$ converges to a vertical catenoid with waist circle of radius $\frac{1}{2 \pi}$. When $F$ tends to horizontal, one can shrink $M_{\lambda(F)}$ so that $F$ tends 


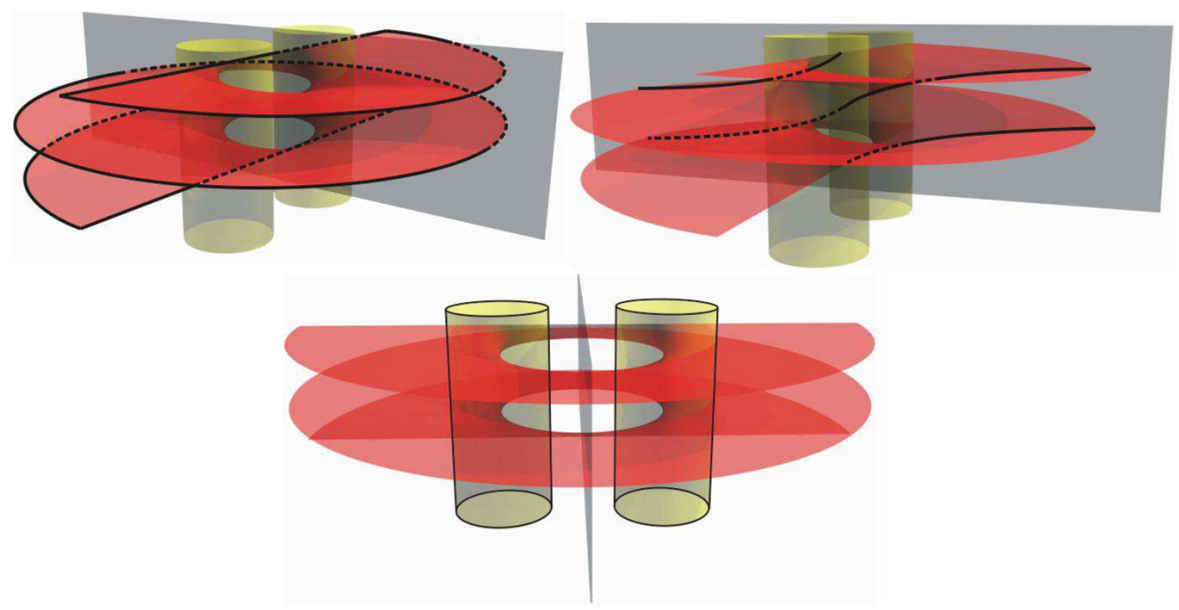

FiguRE 11. Three views of the same Riemann minimal example, with large horizontal flux and two oppositely handed vertical helicoids forming inside solid almost vertical cylinders, one at each side of the vertical plane of symmetry.

to $(4,0,0)$, and in that case the $M_{\lambda(F)}$ converge to the foliation of $\mathbb{R}^{3}$ by horizontal planes, outside of the two vertical lines $\left\{\left(0, \pm 1, x_{3}\right) \mid x_{3} \in \mathbb{R}\right\}$, along which the surface $M_{\lambda(F)}$ approximates two oppositely handed, highly sheeted, scaled-down vertical helicoids; see Figures 11 and 12. With this basic family of examples in mind, we state the following result by Colding and Minicozzi.

Theorem 4.7 (Limit Lamination Theorem for Planar Domains [24, 111]). Let $M_{n} \subset \mathbb{B}\left(R_{n}\right)$ be a locally simply connected sequence of embedded minimal planar domains with $\partial M_{n} \subset \partial \mathbb{B}\left(R_{n}\right), R_{n} \rightarrow \infty$, such that $M_{n} \cap \mathbb{B}(2)$ contains a component which is not a disk for any $n$. If $\sup \left|K_{M_{n} \cap \mathbb{B}(1)}\right| \rightarrow \infty$, then there exists a subsequence of the $M_{n}$ (denoted in the same way) and two vertical lines $S_{1}, S_{2}$, such that:

(a) $M_{n}$ converges away from $S_{1} \cup S_{2}$ to the foliation $\mathcal{F}$ of $\mathbb{R}^{3}$ by horizontal planes.

(b) Away from $S_{1} \cup S_{2}$, each $M_{n}$ consists of exactly two multivalued graphs spiraling together. Near $S_{1}$ and $S_{2}$, the pair of multivalued graphs forms two double spiral staircases with opposite handedness at $S_{1}$ and $S_{2}$. Thus, circling only $S_{1}$ or only $S_{2}$ results in going either up or down, while a path circling both $S_{1}$ and $S_{2}$ closes up; see Figure 12.

Theorem 4.7 shows a second example of a limiting parking garage structure on $\mathbb{R}^{3}$ (we obtained the first example in Theorem 4.2 above), now with two columns which are $(+,-)$-handed 35 just as in the case of the Riemann minimal examples $M_{\lambda}$ discussed before the statement of Theorem 4.7. We refer the reader to [116] for more details about parking garage structures on $\mathbb{R}^{3}$.

To finish this section, we want to mention that the one-sided curvature estimate of Colding and Minicozzi (Theorem 4.3) can be used to solve an important problem

\footnotetext{
${ }^{35}$ Here + (resp. - $)$ means that the corresponding forming helicoid or multigraph is righthanded (resp. left-handed).
} 

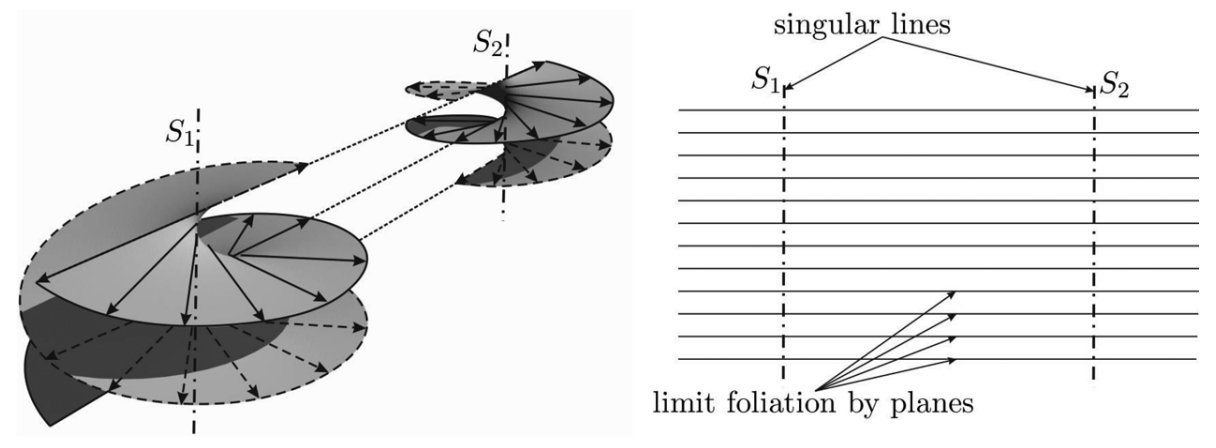

limit foliation by planes

Figure 12. Left: Two oppositely handed double spiral staircases. Right: The limit foliation by parallel planes and the singular set of convergence $S_{1} \cup S_{2}$.

in classical minimal surface theory, known as the Generalized Nitsche Conjecture. In 1962, Nitsche [157] conjectured that if a minimal surface in $\mathbb{R}^{3}$ meets every horizontal plane in a Jordan curve, then it must be a catenoid (he also proved this conjecture with the additional assumption that every horizontal section of the surface is a star-shaped curve). In 1993, Meeks and Rosenberg [133] showed that if a properly embedded minimal surface $M \subset \mathbb{R}^{3}$ has at least two ends, then any annular end $E \subset M$ either has finite total curvature or it satisfies the hypotheses of the following conjecture.

Conjecture 4.8 (Generalized Nitsche Conjecture, Collin's Theorem [38]). Let E $\subset$ $\left\{x_{3} \geq 0\right\}$ be a properly embedded minimal annulus with $\partial E \subset\left\{x_{3}=0\right\}$, such that $E$ intersects each plane $\left\{x_{3}=t\right\}, t>0$, in a simple closed curve. Then, $E$ has finite total curvature, and so, $E$ is asymptotic to a plane or to an end of a catenoid.

This problem was originally solved by Collin [38] before the Colding-Minicozzi results, with a beautiful and long proof. Later on, Colding and Minicozzi gave an alternative proof of Conjecture 4.8. as a short application of Theorem 4.3 that can be found in detail in [26] and in a survey by Rosenberg [182]. We will only state here a consequence of the one-sided curvature estimate by Colding and Minicozzi, which implies directly the Generalized Nitsche Conjecture. Given $\varepsilon \in \mathbb{R}$, we denote by $\mathcal{C}_{\varepsilon}$ the conical region $\left\{x_{3}>\varepsilon \sqrt{x_{1}^{2}+x_{2}^{2}}\right\}$.

Theorem 4.9 (Colding, Minicozzi [26]). There exists a $\delta>0$ such that any properly embedded minimal annular end $E \subset \mathcal{C}_{-\delta}$ has finite total curvature.

4.2. Examples of minimal laminations with isolated singularities. A fundamental theoretical question deals with finding appropriate conditions under which a minimal lamination of a three-manifold $N$ minus a closed subset $A \subset N$ extends across $A$. In this section, we first describe examples in $\mathbb{B}(1)=\mathbb{B}(\overrightarrow{0}, 1) \subset \mathbb{R}^{3}$ with the origin as the unique non-removable singularity. We then indicate how these examples lead to related singular minimal laminations in the homogeneous spaces $\mathbb{H}^{3}$ and $\mathbb{H}^{2} \times \mathbb{R}$ (here $\mathbb{H}^{n}$ denotes the $n$-dimensional hyperbolic space)

ExAmple I. Catenoid type laminations. Consider the sequence of horizontal circles $C_{n}=\mathbb{S}^{2} \cap\left\{x_{3}=\frac{1}{n}\right\}, n \geq 2$. Note that each pair $C_{2 k}, C_{2 k+1}$ bounds a compact unstable piece of a catenoid $M(k)$ and that 

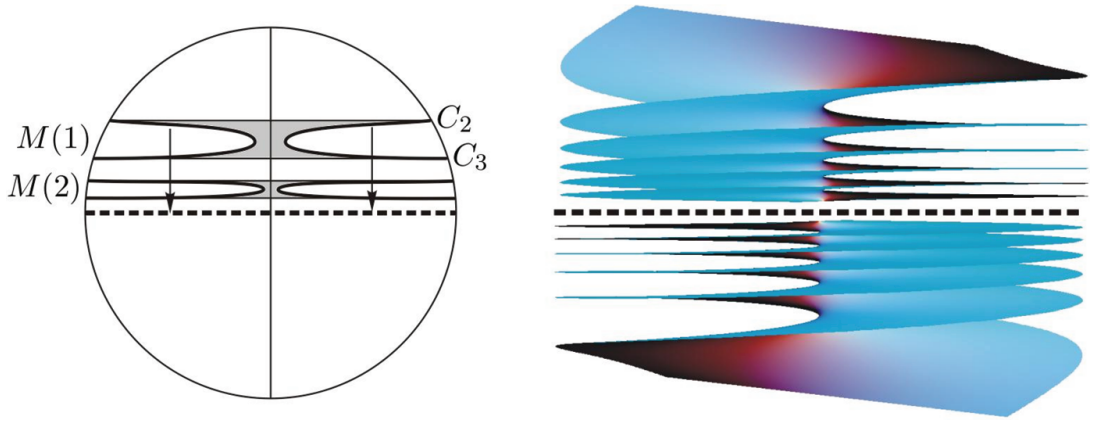

Figure 13. Left: A catenoid type lamination, which explains why the hypothesis of simply connectedness of Theorem 4.3 is necessary. Right: A Colding-Minicozzi type lamination in a cylinder.

$M(k) \cap M\left(k^{\prime}\right)=\varnothing$ if $k \neq k^{\prime}$. The sequence $\{M(k)\}_{k}$ converges with multiplicity two outside the origin $\overrightarrow{0}$ to the closed horizontal disk $\overline{\mathbb{D}}$ of radius 1 centered at $\overrightarrow{0}$. Thus, $\{M(k)-\partial M(k)\}_{k} \cup\{\mathbb{D}-\{\overrightarrow{0}\}\}$ is a minimal lamination of $\mathbb{B}(1)-\{\overrightarrow{0}\}$, which does not extend through the origin; see Figure 13, left.

Example II. Colding-Minicozzi examples. In their paper [29], Colding and Minicozzi constructed a sequence of compact, embedded minimal disks $D_{n} \subset \mathbb{B}(1)$ with boundary in $\mathbb{S}^{2}$ that converge to a singular minimal lamination $\overline{\mathcal{L}}$ of the closed ball $\overline{\mathbb{B}}(\overrightarrow{0}, 1)$ which has an isolated singularity at $\overrightarrow{0}$. The related lamination $\mathcal{L}$ of $\mathbb{B}(1)-\{\overrightarrow{0}\}$ consists of a unique limit leaf which is the punctured open disk $\mathbb{D}-\{\overrightarrow{0}\}$, together with two non-proper leaves that spiral into $\mathbb{D}-\{\overrightarrow{0}\}$ from opposite sides; see Figure 13 , right.

Consider the exhaustion of $\mathbb{H}^{3}$ (naturally identified with $\mathbb{B}(1)$ through the Poincaré model) by hyperbolic balls of hyperbolic radius $n$ centered at the origin, together with compact minimal disks with boundaries on the boundaries of these balls, similar to the compact Colding-Minicozzi disks. We conjecture that these examples produce a similar limit lamination of $\mathbb{H}^{3}-\{\overrightarrow{0}\}$ with three leaves, one of which is totally geodesic and the other two of which are not proper and that spiral into the first one. We remark that one of the main results of the Colding-Minicozzi theory, Theorem 4.2 above, insures that such an example cannot be constructed in $\mathbb{R}^{3}$.

Example III. Catenoid-type laminations in $\mathbb{H}^{3}$ and in $\mathbb{H}^{2} \times \mathbb{R}$. Consider the same circles $C_{n}$ as in Example I, where $\mathbb{S}^{2}$ is now viewed as the boundary at infinity of $\mathbb{H}^{3}$. Then each pair of circles $C_{2 k}, C_{2 k+1}$ is the asymptotic boundary of a properly embedded, unstable minimal annulus $M(k)$, which is a surface of revolution called a catenoid. The sequence $\{M(k)\}_{k}$ converges with multiplicity two outside of $\overrightarrow{0}$ to the horizontal, totally geodesic subspace $\mathbb{D}$ at height zero. Thus, $\{M(k)-\partial M(k)\}_{k} \cup\{\mathbb{D}-\{\overrightarrow{0}\}\}$ is a minimal lamination of 
$\mathbb{H}^{3}-\{\overrightarrow{0}\}$, which does not extend through the origin. A similar catenoidal construction can be done in $\mathbb{H}^{2} \times \mathbb{R}$, where we consider $\mathbb{H}^{2}$ with the Poincaré disk model of the hyperbolic plane. Note that the Half-space Theorem 2.17 excludes this type of singular minimal lamination in $\mathbb{R}^{3}$.

\section{The structure of minimal laminations of $\mathbb{R}^{3}$}

The goal of this section is to give a description of the structure of a general minimal lamination of $\mathbb{R}^{3}$. This description is a crucial tool to study limits of embedded minimal surfaces, and it will be used in the proofs of Theorems 1.1 and 1.2 ,

In his beautiful survey on minimal surfaces, Rosenberg 182 introduced the subject of his paper (and of this section) through a question asked to him by Haefliger about thirty years ago: Is there a foliation of $\mathbb{R}^{3}$ by minimal surfaces, other than a foliation by parallel planes?

Any leaf $L$ of a minimal foliation of $\mathbb{R}^{3}$ is a complete limit leaf, and by Theorem 4.5 it is stable. Now Theorem 2.25 implies that $L$ is a plane. Thus, the answer to Haefliger's question is no. Immediately one is tempted to extend this question to minimal laminations.

Question 5.1. What are the minimal laminations of $\mathbb{R}^{3}$ ?

There are only two known types of minimal laminations of $\mathbb{R}^{3}$, both rather simple: a lamination with exactly one leaf which is a properly embedded minimal surface, or a lamination consisting of a closed set of parallel planes. Rosenberg [136] has conjectured that these are the unique possible examples. To the contrary, Meeks has conjectured that there exists a minimal lamination $\mathcal{L}$ of $\mathbb{R}^{3}$ with two planar leaves and a non-flat leaf which is proper in the open slab between these two planes in $\mathcal{L}$. Since every leaf of a minimal lamination of $\mathbb{R}^{3}$ is complete, the above question is closely related to the following one, known as the embedded Calabi-Yau problem in $\mathbb{R}^{3}$ (see also Conjecture 11.20):

Question 5.2. When is a complete, embedded minimal surface $M \subset \mathbb{R}^{3}$ proper?

Given a minimal lamination $\mathcal{L}$ of $\mathbb{R}^{3}$, the function that assigns to each point $p$ in $\mathcal{L}$ the Gaussian curvature of the leaf $L \in \mathcal{L}$ passing through $p$ is continuous in the subspace topology. Since the intersection of $\mathcal{L}$ with any closed ball is compact, we conclude that the intersection of any leaf $L \in \mathcal{L}$ with a ball has Gaussian curvature bounded from below by a constant that only depends on the ball (in other words, $\mathcal{L}$ has locally bounded Gaussian curvature). Reciprocally, if $M$ is a complete, embedded minimal surface in $\mathbb{R}^{3}$ with locally bounded Gaussian curvature, then the closure $\bar{M}$ of $M$ is a minimal lamination of $\mathbb{R}^{3}$ (Lemma 1.1 in [136]). With this perspective, it is natural to study complete, embedded minimal surfaces $M \subset \mathbb{R}^{3}$ with locally bounded Gaussian curvature, as a first stage for answering Questions 5.1 and 5.2 If $M$ is such a minimal surface and it is not proper, then $\bar{M}-M$ may or may not be non-empty, but since $M$ has locally bounded curvature, $\mathcal{L}=\bar{M}$ is a non-trivial minimal lamination of $\mathbb{R}^{3}$. Since $M$ is not proper, then some leaf $L \in \mathcal{L}$ must be a limit leaf, and hence stable by Theorem 4.5. Now an argument similar to the one we used to answer Haefliger's question at the beginning of this section insures that $L$ is a plane; so in this case, $\bar{M}-M$ is always non-empty. This can be stated as follows. 
Lemma 5.3 (Meeks, Rosenberg [136]). Let $M \subset \mathbb{R}^{3}$ be a connected, complete, embedded minimal surface with locally bounded Gaussian curvature. Then, exactly one of the following statements holds:

(1) $M$ is properly embedded in $\mathbb{R}^{3}$.

(2) $M$ is properly embedded in an open half-space, with limit set consisting of the boundary plane of this half-space.

(3) $M$ is properly embedded in an open slab, with limit set consisting of the boundary planes of this slab.

It should be mentioned that in a previous work, Xavier 209] proved that a complete, immersed, non-flat minimal surface of bounded curvature in $\mathbb{R}^{3}$ cannot be contained in a half-space. This result together with Lemma 5.3 gives a partial answer to Question 5.2 .

Corollary 5.4 (Meeks, Rosenberg [136]). If $M \subset \mathbb{R}^{3}$ is a connected, complete, embedded minimal surface with bounded Gaussian curvature, then $M$ is proper.

The next step in the study of complete, embedded, non-proper minimal surfaces consists of understanding how they accumulate to the limit set described in Lemma 5.3

Lemma 5.5 (Meeks, Rosenberg [136). Let $M \subset \mathbb{R}^{3}$ be a connected, complete, embedded minimal surface with locally bounded Gaussian curvature. Suppose that $M$ is not proper and let $\Pi$ be a limit plane of $M$. Then, for any $\varepsilon>0$, the closed $\varepsilon$-neighborhood of $\Pi$ intersects $M$ in a path connected set.

Outline of the proof. The argument is by contradiction. Without loss of generality, we may assume that $\Pi=\left\{x_{3}=0\right\}$ and that $M$ limits to $\Pi$ from above. Assuming that the statement fails, one can find an $\varepsilon>0$ and a stable minimal surface $\Sigma$ between two components of the intersection of $M$ with the slab $\left\{0<x_{3} \leq \varepsilon\right\}$ by the usual barrier construction argument discussed in Section 2.9. Since $\Sigma$ satisfies curvature estimates away from its boundary (Theorem 2.27), we conclude that for sufficiently small $\delta \in(0, \varepsilon)$, the orthogonal projection $\pi$ to $\Pi$, restricted to a component $\Sigma(\delta)$ of $\Sigma \cap\left\{0<x_{3}<\delta\right\}$, is a local diffeomorphism. A topological argument shows that $\left.\pi\right|_{\Sigma(\delta)}$ is in fact a diffeomorphism between $\Sigma(\delta)$ and its image. This implies that $\Sigma(\delta)$ is a graph, and so, it is properly embedded in the slab $\left\{0 \leq x_{3} \leq \delta\right\}$. By Theorem 7.7 below, $\Sigma(\delta)$ is a parabolic surface but $\left.x_{3}\right|_{\Sigma(\delta)}$ is a non-constant, bounded harmonic function with constant boundary value $\delta$, which gives a contradiction and proves Lemma 5.5 .

A refinement of the argument in the previous paragraph gives the following result.

Lemma 5.6 (Meeks, Rosenberg 136]). Let $M \subset \mathbb{R}^{3}$ be a connected, complete, nonproper, embedded minimal surface with locally bounded Gaussian curvature, which limits to the plane $\Pi=\left\{x_{3}=0\right\}$ from above. Then, for any $\varepsilon>0$, the Gaussian curvature of $M \cap\left\{0<x_{3} \leq \varepsilon\right\}$ is not bounded from below.

Let $M \subset \mathbb{R}^{3}$ satisfy the hypotheses of Lemma 5.6. Then, there exists a sequence $\left\{p_{n}\right\}_{n} \subset M$ such that $x_{3}\left(p_{n}\right) \searrow 0$ and the curvature function $K_{M}$ of $M$ satisfies $\left|K_{M}\left(p_{n}\right)\right| \rightarrow \infty$ as $n$ goes to infinity. Such a sequence must diverge in space because $K_{M}$ is locally bounded. If we additionally assume $M$ has finite topology, then an application of the Colding-Minicozzi one-sided curvature estimate (Theorem 4.3) 
contradicts that $\left|K_{M}\left(p_{n}\right)\right| \rightarrow \infty$. This is a rough sketch of the proof of the following statement.

Theorem 5.7 (Meeks, Rosenberg [136]). If $M \subset \mathbb{R}^{3}$ is a connected, complete, embedded minimal surface in $\mathbb{R}^{3}$ with finite topology and locally bounded Gaussian curvature, then $M$ is proper.

Meeks, Pérez and Ros (Theorem 5 in 126]) have combined the last statement with deeper arguments using the results of Colding and Minicozzi, which let us exchange the finite topology assumption by the weaker hypothesis of finite genus.

Theorem 5.8 (Meeks, Pérez, Ros [126]). If $M \subset \mathbb{R}^{3}$ is a connected, complete, embedded minimal surface in $\mathbb{R}^{3}$ with finite genus and locally bounded Gaussian curvature, then $M$ is proper.

In conclusion, we state the following descriptive result for minimal laminations of $\mathbb{R}^{3}$, by Meeks and Rosenberg [136] as generalized by Meeks, Pérez and Ros [126].

Theorem 5.9 (Structure Theorem for Minimal Laminations of $\mathbb{R}^{3}$ ). For a given minimal lamination $\mathcal{L}$ of $\mathbb{R}^{3}$, one of the following possibilities holds.

i) $\mathcal{L}$ has exactly one leaf, which consists of a properly embedded minimal surface in $\mathbb{R}^{3}$.

ii) $\mathcal{L}$ has more than one leaf and consists of the disjoint union of a nonempty closed set of parallel planes $\mathcal{P} \subset \mathcal{L}$ together with a collection of complete minimal surfaces of unbounded Gaussian curvature and infinite genus, which are properly embedded in the open slabs and half-spaces of $\mathbb{R}^{3}-\mathcal{P}$. Furthermore, each of the open slabs and half-spaces in $\mathbb{R}^{3}-\mathcal{P}$ contains at most one leaf of $\mathcal{L}$, and every plane parallel to but different from the planes in $\mathcal{P}$ intersects at most one of the leaves of $\mathcal{L}$ and separates such an intersecting leaf into exactly two components.

There are no known examples of laminations of $\mathbb{R}^{3}$ as described in item ii) of the last theorem; see item 1 in Conjecture 11.20.

\section{THE ORDERING THEOREM FOR THE SPACE OF ENDS}

The study of the ends of a properly embedded minimal surface $M \subset \mathbb{R}^{3}$ with more than one end has been extensively developed. Callahan, Hoffman and Meeks [16] showed that in one of the closed complements of $M$ in $\mathbb{R}^{3}$, there exists a non-compact, properly embedded minimal surface $\Sigma$ with compact boundary and finite total curvature. By the discussion following Theorem 2.11, the ends of $\Sigma$ are of catenoidal or planar type, and the embeddedness of $\Sigma$ forces its ends to have parallel normal vectors at infinity.

Definition 6.1. In the above situation, the limit tangent plane at infinity of $M$ is the plane in $\mathbb{R}^{3}$ passing through the origin, whose normal vector equals (up to sign) the limiting normal vector at the ends of $\Sigma$. Such a plane is unique [16], in the sense that it does not depend on the finite total curvature minimal surface $\Sigma \subset \mathbb{R}^{3}-M$.

The limit tangent plane at infinity is a key notion for studying the way in which a minimal surface with more than one end embeds properly in space.

Theorem 6.2 (Ordering Theorem, Frohman, Meeks 61]). Let $M \subset \mathbb{R}^{3}$ be a properly embedded minimal surface with more than one end and horizontal limit 
tangent plane at infinity. Then, the space $\mathcal{E}(M)$ of ends of $M$ is linearly ordered geometrically by the relative heights of the ends over the $\left(x_{1}, x_{2}\right)$-plane, and embeds topologically in $[0,1]$ in an ordering preserving way. Furthermore, this ordering has a topological nature in the following sense: If $M$ is properly isotopic to a properly embedded minimal surface $M^{\prime}$ with horizontal limit tangent plane at infinity, then the associated ordering of the ends of $M^{\prime}$ either agrees with or is opposite to the ordering coming from $M$.

Given a minimal surface $M \subset \mathbb{R}^{3}$ satisfying the hypotheses of Theorem 6.2, we define the top end $e_{T}$ of $M$ as the unique maximal element in $\mathcal{E}(M)$ for the ordering given in this theorem (recall from Section 2.7 that $\mathcal{E}(M) \subset[0,1]$ is compact; hence $e_{T}$ exists). Analogously, the bottom end $e_{B}$ of $M$ is the unique minimal element in $\mathcal{E}(M)$. If $e \in \mathcal{E}(M)$ is neither the top nor the bottom end of $M$, then it is called a middle end of $M$.

Rather than sketching the proof of the Ordering Theorem, we will be content to explain how one obtains the linear ordering. Suppose $M \subset \mathbb{R}^{3}$ is a minimal surface in the hypotheses of Theorem 6.2 and let $\mathcal{A} \subset \mathcal{E}(M)$ be the set of annular ends of $M$. In this setting, Collin's Theorem 1.3 insures that every proper annular representative of an end $e \in \mathcal{A}$ has finite total curvature and thus, it is asymptotic to a horizontal plane or to a half-catenoid. Since the ends in $\mathcal{A}$ are all graphs over complements of compact subdomains in the $\left(x_{1}, x_{2}\right)$-plane, we see that $\mathcal{A}$ has a natural linear ordering by relative heights of its ends over the $\left(x_{1}, x_{2}\right)$-plane. Hence the Ordering Theorem is proved when $\mathcal{A}=\mathcal{E}(M)$.

We next indicate how to extend the above linear ordering to $\mathcal{E}(M)-\mathcal{A}$. By Theorem 2.28, any end of $M$ which can be represented by a proper subdomain that is stable can also be represented by a surface of finite total curvature and so, it can be represented by an annulus. Let $e_{1}=\left[\alpha_{1}\right] \in \mathcal{E}(M)$ be an end which is not annular. Such an end can always be represented by a proper subdomain $E_{1} \subset M$ with the following two properties (recall we are assuming $M$ has at least two ends):

- $E_{1}$ is unstable, and $\partial E_{1}$ is compact and connected.

- $\overline{M-E_{1}}$ is unstable and non-compact.

Let $W_{1}, W_{2}$ be the two closed complements of $M$ in $\mathbb{R}^{3}$. Note that we can consider $E_{1}$ to lie on the boundary of both of these complete flat three-manifolds $W_{1}, W_{2}$, and that their boundaries $\partial W_{1}, \partial W_{2}$ are good barriers for solving Plateau-type problems. Since $E_{1}$ and $\overline{M-E_{1}}$ are both non-compact, elementary separation properties for proper surfaces in $\mathbb{R}^{3}$ imply that $\partial E_{1}$ is not homologous to zero in one of the domains $W_{1}, W_{2}$; suppose that $\partial E_{1}$ is not homologous to zero in $W_{1}$. Since $\partial E_{1}$ bounds the locally finite 2 -chain $E_{1}$ in $\partial W_{1}$, the barrier argument in Section 2.9 shows that $\partial E_{1}$ is the boundary of a properly embedded, orientable, least-area surface $\Sigma_{1}$ in $W_{1}$, which is non-compact since $\partial E_{1}$ is not homologous to zero in $W_{1}$. Similarly, $\partial E_{1}$ is the boundary of a least-area (possibly compact) surface $\Sigma_{2}$ in $W_{2}$. Since $E_{1}$ and $M-E_{1}$ are unstable, the maximum principle implies that $\left(\Sigma_{1} \cup \Sigma_{2}\right) \cap M=\partial E_{1}$; see Figure 14 .

Let $R_{1}$ be the closed complement of $\Sigma_{1} \cup \Sigma_{2}$ in $\mathbb{R}^{3}$ which contains $E_{1}$ and let $R_{2}$ be the other closed complement. Since $E_{1}$ and $\overline{M-E_{1}}$ are both non-compact and $M$ is properly embedded in $\mathbb{R}^{3}$, then $R_{1}$ and $R_{2}$ are both non-compact. It follows from Theorem 2.28 that outside a large ball containing $\partial E_{1}$, the boundary of $R_{1}$, which equals $\Sigma_{1} \cup \Sigma_{2}=\partial R_{2}$, consists of a finite positive number of graphical ends which are asymptotic to ends of horizontal planes and vertical catenoids. Let 


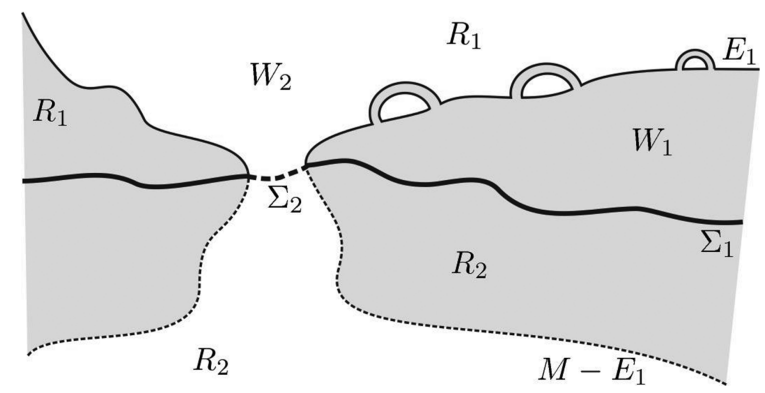

Figure 14. The least-area surfaces $\Sigma_{1}, \Sigma_{2} . W_{1}$ is the shaded region, and $R_{1}$ lies above $\Sigma_{1} \cup \Sigma_{2}$.

$e_{2}=\left[\alpha_{2}\right] \in \mathcal{E}(M)$ be an end with a proper subdomain representative $E_{2} \subset M$ which is disjoint from $E_{1}$ (note that any two distinct ends can be chosen to have disjoint representatives). Next we define when $\left[\alpha_{1}\right]<\left[\alpha_{2}\right]$ or vice versa in the linear ordering of Theorem 6.2. The proper $\operatorname{arcs} \alpha_{1}, \alpha_{2}$ which represent $e_{1}, e_{2}$ eventually lie in $R_{1}, R_{2}$ and so, exactly one of the following cases holds:

- $\alpha_{1}$ eventually lies in the open region between two successive graphical ends of $\partial R_{1}$, and $\alpha_{2}$ eventually lies above or below this region.

- $\alpha_{1}$ eventually lies in the open region above the top graphical end of $\partial R_{1}$, and $\alpha_{2}$ eventually lies below this region.

- $\alpha_{1}$ eventually lies in the open region below the bottom graphical end of $\partial R_{1}$, and $\alpha_{2}$ eventually lies above this region.

In particular, there is a topological plane $P$ which is a graph over the $\left(x_{1}, x_{2}\right)$-plane and whose end is one of the ends of $\partial R_{1}$, such that eventually $\alpha_{1}$ and $\alpha_{2}$ lie at opposite sides of $P$. If $\alpha_{1}$ eventually lies below $P$ and $\alpha_{2}$ eventually lies above $P$, then $\left[\alpha_{1}\right]<\left[\alpha_{2}\right]$ in the linear ordering given by the Ordering Theorem; otherwise, $\left[\alpha_{2}\right]<\left[\alpha_{1}\right]$. The ordering we have just described can be proven to be a well-defined linear ordering; see [61] for more details.

\section{Conformal structure of minimal surfaces}

7.1. Recurrence and parabolicity for manifolds. The conformal structure of a complete minimal surface has a strong influence on its global properties. For this reason, an important conformal question is to decide the so-called type problem for a minimal surface $M$ in $\mathbb{R}^{3}$, in the sense of classical Riemann surfaces, i.e., whether $M$ is hyperbolic or paraboli 36 (the elliptic or compact case is impossible for such a minimal surface by the maximum principle for harmonic functions). It turns out that parabolicity for Riemann surfaces without boundary is equivalent to the recurrence of Brownian motion on such surfaces. This field lies in the borderline between several branches of mathematics such as Riemannian geometry, stochastic analysis, partial differential equations and potential theory. A particularly interesting source,

\footnotetext{
${ }^{36}$ Classically, a Riemann surface without boundary is called hyperbolic if it carries a nonconstant positive superharmonic function, and parabolic if it is neither elliptic (i.e., compact) nor hyperbolic. The reader should be aware that we will use the concept of parabolicity for Riemannian manifolds with boundary (see Definition 7.1) and reserve the word recurrent for manifolds without boundary (Definition 7.2). For Riemannian manifolds, the relationship between parabolicity and recurrence will become clear soon.
} 
where the reader can find an excellent introduction to these questions, is the survey of recurrence and Brownian motion on Riemannian manifolds by Grigor'yan [66.

The goal of this section is to introduce some key concepts which are useful when dealing with these conformal questions. Instead of using concepts related to probability (such as random walks or Brownian motion), in this paper we will follow an alternative way to define recurrence and parabolicity that is slightly different from Grigor'yan's approach; our approach is well known and is explained in greater detail in the notes by the second author [166]. Most of the results stated in this section are proved in [66] or [166].

Definition 7.1. Let $\left(M^{n}, g\right)$ be an $n$-dimensional Riemannian manifold with nonempty boundary. $M$ is parabolic if every bounded harmonic function on $M$ is determined by its boundary values.

Definition 7.2. Let $\left(M^{n}, g\right)$ be an $n$-dimensional Riemannian manifold without boundary. $M$ is recurrent if for any non-empty open set $U \subset M(U \neq M)$ with smooth boundary, $M-U$ is parabolic. $M$ is called transient if it is not recurrent.

Given a Riemannian manifold $(M, g)$ with boundary $\partial M \neq \varnothing$ and a point $p \in$ $\operatorname{Int}(M)$, the harmonic measure $\mu_{p}$ with respect to $p$ can be defined as follows. Let $I \subset \partial M$ be a non-empty open set with smooth boundary. Consider a compact exhaustion $M_{1} \subset M_{2} \subset \cdots$ of $M$. Given $k \in \mathbb{N}$, let $h_{k}: M_{k} \rightarrow[0,1]$ be the (bounded) harmonic function on $M_{k}$ with boundary values 1 on the interior of $I \cap M_{k}$ and 0 on $\partial M_{k}-\bar{I}$. After extending $h_{k}$ by zero to $M$, we can view $\left\{h_{k}\right\}_{k}$ as an increasing sequence of subharmonic functions, bounded from above by 1 . The functions $h_{k}$ limit to a unique bounded harmonic function $h_{I}: M \rightarrow[0,1]$ (defined except at countably many points in $\partial I \subset \partial M$ ). In this situation, we define $\mu_{p}(I)=h_{I}(p)$. It turns out that $\mu_{p}$ extends to a Borel measure $\mu_{p}$ on $\partial M$. For another interpretation of $\mu_{p}$ related to Brownian motion, see Grigor'yan [66] and also [116].

Parabolicity and harmonic measure are closely related, as states the following elementary result.

Proposition 7.3. Let $(M, g)$ be a Riemannian manifold with $\partial M \neq \varnothing$. Then, the following statements are equivalent:

1. $M$ is parabolic.

2. There exists a point $p \in \operatorname{Int}(M)$ such that the harmonic measure $\mu_{p}$ is full; i.e., $\int_{\partial M} \mu_{p}=1$.

3. Given any $p \in \operatorname{Int}(M)$ and any bounded harmonic function $f: M \rightarrow \mathbb{R}$, then $f(p)=\int_{\partial M} f \mu_{p}$.

4. The universal covering of $M$ is parabolic.

Furthermore, if there exists a proper, non-negative superharmonic function on $M$, then $M$ is parabolic. When $M$ is simply connected and two-dimensional, then the existence of such a function is equivalent to being parabolic.

We note that the parabolicity of a Riemannian manifold with boundary is not affected by adding compact sets or by removing interiors of compact sets, and if a manifold $M$ can be decomposed as the union of two parabolic domains with compact intersection, then $M$ is parabolic (or recurrent, depending on whether $\partial M$ is empty or not). Since the closed unit disk $\overline{\mathbb{D}}$ is parabolic and $x \in \mathbb{R}^{2}-\mathbb{D} \mapsto$ $\log |x|$ is a proper, non-negative harmonic function, then it follows that $\mathbb{R}^{2}-\mathbb{D}$ is 
parabolic and $\mathbb{R}^{2}$ is recurrent. On the other hand, for $n \in \mathbb{N}, n \geq 3$, the function $x \in \mathbb{R}^{n}-\{|x|<1\} \mapsto|x|^{2-n}$ is bounded and harmonic with constant boundary values. So, $\mathbb{R}^{n}-\{|x|<1\}$ is not parabolic and $\mathbb{R}^{n}$ is transient if $n \geq 3$.

Note that if $h: M \rightarrow \mathbb{R}$ is a non-constant, positive harmonic function on a recurrent Riemannian manifold, then for any positive regular value $t \in \mathbb{R}$ of $h$, the closed subset $M_{t}=h^{-1}((0, t])$ is parabolic and $\left.h\right|_{M_{t}}$ is a bounded harmonic function with constant boundary value $t$. By Proposition $7.3,\left.h\right|_{M_{t}}$ is the constant function $t$, which contradicts that $t$ is a regular value of $h$. This contradiction completes the proof of the following well-known result.

Proposition 7.4 (Liouville Theorem). Every positive harmonic function on a recurrent Riemannian manifold is constant.

7.2. Universal superharmonic functions and parabolicity of minimal surfaces. As we mentioned before, the knowledge of the conformal type of a minimal surface $M$ is crucial when tackling uniqueness questions. Sometimes it is useful to decompose $M$ in pieces and study the conformal structure of each piece as a Riemann surface with boundary. For instance, the proof by Meeks and Rosenberg of the uniqueness of the helicoid rests on the non-trivial fact 37 that a simply connected, properly embedded minimal surface $M \subset \mathbb{R}^{3}$ must admit a plane which intersects $M$ transversely in a single proper arc $\gamma$. Each of the two closed complements of $\gamma$ in $M$ is contained in a closed half-space. Hence, from Theorem 7.7 below, both halves are parabolic, and one then uses this information to prove that $M$ is conformally $\mathbb{C}$. A key ingredient in the proof of Theorem 7.7 is the construction of a function defined on a certain region of space, whose restriction to any minimal surface contained in that region is superharmonic. This construction leads us to the following definition.

Definition 7.5. Given a region $W \subset \mathbb{R}^{3}$, a function $h: W \rightarrow \mathbb{R}$ is said to be a universal superharmonic function on $W$ if its restriction to any minimal surface $M \subset W$ is superharmonic.

Examples of universal superharmonic functions on all of $\mathbb{R}^{3}$ include coordinate functions such as $x_{1}$ or the function $-x_{1}^{2}$. Collin, Kusner, Meeks and Rosenberg proved the following useful inequality (Lemma 2.2 in 39]) valid for any immersed minimal surface in $\mathbb{R}^{3}$ :

$$
|\Delta \ln r| \leq \frac{\left|\nabla x_{3}\right|^{2}}{r^{2}} \quad \text { in } M-\left(x_{3} \text {-axis }\right),
$$

where $r=\sqrt{x_{1}^{2}+x_{2}^{2}}$ and $\nabla, \Delta$ denote the intrinsic gradient and Laplacian on $M$. Using the estimate (12), it is easy to check that the following statements hold.

\section{Lemma 7.6.}

i) The function $\ln r-x_{3}^{2}$ is a universal superharmonic function in the region $\left\{r^{2} \geq \frac{1}{2}\right\}$.

ii) The function $\ln r-x_{3} \arctan x_{3}+\frac{1}{2} \ln \left(x_{3}^{2}+1\right)$ is a universal superharmonic function in the region $\left\{r^{2} \geq x_{3}^{2}+1\right\}$.

Let $M$ be a properly immersed minimal surface in a closed half-space. If $\partial M=$ $\varnothing$, then $M$ is planar by the Half-space Theorem 2.17 (and hence, $M$ is recurrent);

\footnotetext{
${ }^{37}$ See the third paragraph in the sketch of the proof of Theorem 1.1 in Section 8
} 
if $\partial M \neq \varnothing$, then $M$ is parabolic by a direct application of the following general theorem.

Theorem 7.7 (Collin, Kusner, Meeks, Rosenberg [39]). Let $M$ be a connected, nonplanar, properly immersed minimal surface in $\mathbb{R}^{3}$, possibly with boundary. Then, every component of the intersection of $M$ with a closed half-space is a parabolic surface with boundary. In particular, if $M$ has empty boundary and intersects some plane in a compact set, then $M$ is recurrent.

Proof. Up to a rotation, it suffices to check that any component $C$ of $M(+)=$ $M \cap\left\{x_{3} \geq 0\right\}$ is parabolic. For fixed $n \in \mathbb{N}$, let $C_{n}=C \cap x_{3}^{-1}([0, n])$. By part i) of Lemma 7.6 the function $h=\ln r-x_{3}^{2}$ is superharmonic and proper when restricted to $C_{n} \cap\left\{r^{2} \geq \frac{1}{2}\right\}$. Furthermore, $h$ is positive outside a compact domain on $C_{n}$, which by Proposition 7.3 implies that $C_{n} \cap\left\{r^{2} \geq \frac{1}{2}\right\}$ is parabolic. Since $M$ is proper and $\left\{r^{2} \leq \frac{1}{2}\right\} \cap\left\{0 \leq x_{3} \leq n\right\}$ is compact, we deduce that $C_{n}-\left\{r^{2}>\frac{1}{2}\right\}$ is a compact subset of $C_{n}$. Since parabolicity is not affected by adding compact subsets, it follows that $C_{n}$ is parabolic.

We now check that $C$ is parabolic. Fix a point $p \in C$ with $x_{3}(p)>0$ and let $\mu_{p}^{C}$ be the harmonic measure of $C$ with respect to $p$. For $n$ large enough, $p$ lies in the interior of $C_{n}$. Since $x_{3}$ is a bounded harmonic function on the parabolic surface $C_{n}$, part 3 of Proposition 7.3 insures that

$$
x_{3}(p)=\int_{\partial C_{n}} x_{3} \mu_{p}^{n} \geq n \int_{\partial C_{n} \cap x_{3}^{-1}(n)} \mu_{p}^{n},
$$

where $\mu_{p}^{n}$ is the harmonic measure of $C_{n}$ with respect to $p$. Since $\mu_{p}^{n}$ is full on $\partial C_{n}$, it follows that

$$
\int_{\partial C_{n}-x_{3}^{-1}(n)} \mu_{p}^{n}=1-\int_{\partial C_{n} \cap x_{3}^{-1}(n)} \mu_{p}^{n} \geq 1-\frac{x_{3}(p)}{n} \stackrel{(n \rightarrow \infty)}{\longrightarrow} 1 .
$$

Suppose now that $M$ and $N$ are Riemannian manifolds with $M \subset N, \partial$ is a component of $\partial M \cap \partial N, p \in \operatorname{Int}(M)$ and $\mu_{p}^{M}$ and $\mu_{p}^{N}$ are the respective harmonic measures. Then it follows immediately from the definition of harmonic measure that $\int_{\partial} \mu_{p}^{M} \leq \int_{\partial} \mu_{p}^{N} \leq 1$. By letting $M=C_{n}, N=C$ and $\partial=\partial C_{n}-x_{3}^{-1}(n)$, the above-displayed inequality implies that $\lim _{n} \int_{\partial C_{n}-x_{3}^{-1}(n)} \mu_{p}^{C} \geq 1$, from where we conclude that $\int_{\partial C} \mu_{p}^{C}=1$ and the proof is complete.

There are several problems and results about parabolicity of properly immersed minimal surfaces with boundary in $\mathbb{R}^{3}$ (see Conjecture 11.24 below). Concerning these problems, it is worth mentioning the following results due to Neel.

Theorem 7.8. 1. (Neel [155]) Let $M$ be a properly embedded minimal surface of bounded curvature in $\mathbb{R}^{3}$. Then $M$ has no non-constant bounded harmonic functions.

2. (Neel 154) Let $M$ be a non-flat complete, embedded minimal surface with boundary in $\mathbb{R}^{3}$. Assume that, outside of a compact subset of $M$, the Gauss map image of $M$ is contained in an open, hyperbolic subset of $\mathbb{S}^{2}$. Then $M$ is parabolic. In particular, every minimal graph with boundary over a domain in a plane is parabolic. 
7.3. Quadratic area growth and middle ends. In this section, we will state a result by Collin, Kusner, Meeks and Rosenberg that constrains both the geometry and the topology of any properly embedded minimal surface in $\mathbb{R}^{3}$ with more than one end. This theorem has been used in an essential way by Meeks, Pérez and Ros in the proofs of their classification results in Theorems 1.2, 1.6 and 10.3.

The Ordering Theorem in Section 6 represents the first step in trying to understand the geometry of a properly embedded minimal surface in $\mathbb{R}^{3}$ with more than one end. By the proof of the Ordering Theorem, every middle end of such a surface $M \subset \mathbb{R}^{3}$ with horizontal limit tangent plane at infinity can be represented by a proper subdomain $E \subset M$ with compact boundary such that $E$ "lies between two half-catenoids". This means that $E$ is contained in a neighborhood $S$ of the $\left(x_{1}, x_{2}\right)$-plane, $S$ being topologically a slab, whose width grows at most logarithmically with the distance from the origin. The next result shows that a middle end of a properly embedded minimal surface is never a limit end. More precisely:

Theorem 7.9 (Collin, Kusner, Meeks, Rosenberg [39]). Let $M \subset \mathbb{R}^{3}$ be a properly embedded minimal surface with more than one end and horizontal limit tangent plane at infinity. Then, any limit end of $M$ must be a top or bottom end of $M$. In particular, $M$ can have at most two limit ends, each middle end is simple and the number of ends of $M$ is countable. Furthermore, for each middle end $E$ of $M$ there exists a positive integer $m(E)$ such that

$$
\lim _{R \rightarrow \infty} \frac{\operatorname{Area}(E \cap \mathbb{B}(R))}{\pi R^{2}}=m(E) .
$$

The parity of $m(E)$ is called the parity of the middle end $E$.

We refer the interested reader to [116 for an explanation of the proof of Theorem [7.9. We will content ourselves here with pointing out that the universal superharmonic functions mentioned in Lemma 7.6 are used in the proof, in conjunction with the fact that $E$ is trapped between two catenoids, to show that the area of $E$ in balls $\mathbb{B}(R)$ grows quadratically with $R$.

Theorem 7.9 and the Ordering Theorem (Theorem [6) are crucial tools in the topological classification of properly embedded minimal surfaces in $\mathbb{R}^{3}$ by Frohman and Meeks [62. For a detailed discussion, see [116.

We should remark that embeddedness is crucial in showing that the surface $M$ in the last theorem has a countable number of ends, as follows from the construction by Alarcón, Ferrer and Martín [1 of a genus zero, properly immersed minimal surface in $\mathbb{R}^{3}$ whose space of ends is a Cantor set. In particular, every end of their surface is a limit end.

Collin, Kusner, Meeks and Rosenberg [39] were also able to use universal superharmonic functions to control the geometry of properly embedded minimal surfaces with exactly two limit ends. Their proof of the following theorem is motivated by the proof of a similar theorem by Callahan, Hoffman and Meeks [16] in the classical singly periodic setting.

Theorem 7.10 (Collin, Kusner, Meeks, Rosenberg [39]). Let $M \subset \mathbb{R}^{3}$ be a properly embedded minimal surface with two limit ends and horizontal limit tangent plane at infinity. Then there exists a proper collection $\left\{P_{n} \mid n \in \mathbb{Z}\right\}$ of horizontal planes in $\mathbb{R}^{3}$, ordered by heights, such that each plane $P_{n}$ intersects $M$ transversely in a finite number of simple closed curves. Furthermore, the closed slab $S_{n}$ bounded by 
$P_{n} \cup P_{n+1}$ intersects $M$ in a non-compact domain which represents the nth end of $M$. In particular, by Theorem 7.7, $M$ is recurrent.

\section{Uniqueness of The Helicoid I: The PROPER CASE}

The goal of this section is to outline the arguments by Meeks and Rosenberg 136 in their proof of the uniqueness of the helicoid, which is Theorem 1.4 stated in the introduction. We remark that this result is stated under the assumption that the surface in question is proper, instead of merely complete. We will postpone the proof of this more general version (i.e., in the complete setting; see Theorem 1.1) to Section 9.1 .

Sketch of the proof of Theorem 1.1, assuming properness. Let $M$ be a properly embedded, non-flat, simply connected minimal surface in $\mathbb{R}^{3}$. Consider any sequence of positive numbers $\left\{\lambda_{n}\right\}_{n}$ which decays to zero and let $M(n)=\lambda_{n} M$ be the surface scaled by $\lambda_{n}$. By the Limit Lamination Theorem for Disks (Theorem 4.2), a subsequence of these surfaces converges on compact subsets of $\mathbb{R}^{3}$ to a minimal foliation $\mathcal{L}$ of $\mathbb{R}^{3}$ by parallel planes, with singular set of $C^{1}$-convergence $S(\mathcal{L})$ being a Lipschitz curve that can be parametrized by the height over the planes in $\mathcal{L}$. Part of the proof of Theorem 4.2 depends on a unique extension result for the forming multigraphs, which in our case implies that for $n$ large, the almost flat multigraph which starts to form on $M(n)$ near the origin extends all the way to infinity. From this extension result, one can deduce that the limit foliation $\mathcal{L}$ is independent of the sequence $\left\{\lambda_{n}\right\}_{n}$. After a rotation of $M$ and replacement of the $M(n)$ by a subsequence, we can suppose that the $M(n)$ converge $C^{1}$ to the foliation $\mathcal{L}$ of $\mathbb{R}^{3}$ by horizontal planes, on compact subsets outside of the singular set of convergence given by a Lipschitz curve $S(\mathcal{L})$ parameterized by its $x_{3}$-coordinate. Note that this last property implies that $S(\mathcal{L})$ intersects each horizontal plane exactly once.

The next sketched argument shows that $M$ intersects transversely each of the planes in $\mathcal{L}$. After a translation, we may assume that the origin lies in $M$. Since the origin is a singular point of convergence of the $M(n)$ to $\mathcal{L}$ and $S(\mathcal{L})$ is Lipschitz, it follows that $S(\mathcal{L})$ passes through the origin and is contained in the solid cone $\mathcal{C}_{\varepsilon}=\left\{x_{3}^{2} \geq \varepsilon^{2}\left(x_{1}^{2}+x_{2}^{2}\right)\right\}$, where $\varepsilon>0$ only depends on the one-sided curvature estimate in Theorem 4.3. Let $\Delta$ be the solid cylinder $\left\{x_{1}^{2}+x_{2}^{2} \leq 1,\left|x_{3}\right| \leq \varepsilon\right\}$. The two flat horizontal multigraphs $M_{1}(n), M_{2}(n)$ referred to in item 2 of Theorem 4.2 intersect the cylindrical sides of $\partial \Delta$ almost orthogonally along two long spiraling arcs which are multigraphs over the unit circle $\mathbb{S}^{1}$ in the $\left(x_{1}, x_{2}\right)$-plane, possibly together with a finite number of open arcs, starting and finishing at the top (resp. bottom) planar disks of $\partial \Delta$, which are graphs over their projections in $\mathbb{S}^{1}$. Both spirals lie on the compact component $D(n)$ of $M(n) \cap \Delta$ which contains the origin. After a slight perturbation $\Delta(n)$ of $\Delta$ near the top and bottom boundary disks of $\partial \Delta$ and replacing $\Delta$ by $\Delta(n)$, it can be shown that the boundary of $D(n)$ consists of the two spiraling arcs on the boundary of the cylinder together with two arcs which connect them, one on each of the boundary disks in $\partial \Delta(n)$; in this replacement, the new top and bottom disks in $\partial \Delta(n)$ are minimal although not necessarily flat. Without much difficulty, one can extend the top and bottom disks of $\Delta(n)$ to an almost-horizontal, product minimal foliation of $\Delta(n)$ by graphical minimal disks, such that every boundary circle of these (not necessarily planar) disks intersects each of the two spiraling curves in $\partial D(n)$ at a single point. Since at a point of tangency, two minimal surfaces intersect hyperbolically (negative index), 
Morse theory implies that each leaf of the minimal disk foliation of $\Delta(n)$ intersects $D(n)$ transversely in a simple arc. When $n \rightarrow \infty$, these foliations converge to the restricted foliation $\mathcal{L} \cap \Delta$ by flat horizontal disks. This last statement, together with the openness of the Gauss map of the original surface $M$, implies that $M$ is transverse to $\mathcal{L}$, as desired. Therefore, the stereographical projection of the Gauss map $g: M \rightarrow \mathbb{C} \cup\{\infty\}$ can be expressed as $g(z)=e^{f(z)}$ for some holomorphic function $f: M \rightarrow \mathbb{C}$.

The next step in the proof is to check that the conformal structure of $M$ is $\mathbb{C}$ and that its height differential can be written as $d h=d z, z \in \mathbb{C}$. This part of the proof is longer and delicate and depends on a finiteness result for the number of components of a minimal graph over a possibly disconnected, proper domain in $\mathbb{R}^{2}$ with zero boundary values 38 Note that the non-existence of points in $M$ with vertical normal vector implies that the intrinsic gradient of $x_{3}: M \rightarrow \mathbb{R}$ has no zeros on $M$. Through a series of geometric and analytic arguments that use both the double multigraph convergence of the $M(n)$ to $\mathcal{L}$ outside the cone $\mathcal{C}_{\varepsilon}$ and the above finiteness result for graphs over proper domains of $\mathbb{R}^{2}$, one eventually proves that none of the integral curves of $\nabla x_{3}$ are asymptotic to a plane in $\mathcal{L}$ and that every such horizontal plane intersects $M$ transversely in a single proper arc. Then a straightforward argument using Theorem 7.7 implies that $M$ is recurrent, and thus $M$ is conformally $\mathbb{C}$. The non-existence of points in $M$ with vertical normal vector and the connectedness of its horizontal sections force the height differential to be $d h=d x_{3}+i d x_{3}^{*}=d z$ in a conformal parameterization of $M$. In particular, the third coordinate $x_{3}: \mathbb{C} \rightarrow \mathbb{R}$ is $x_{3}(z)=\Re(z)$, the real part of $z$.

To finish the proof, it only remains to determine the Gauss map $g$ of $M$. Recall that we have already indicated how to show that $g(z)=e^{f(z)}$. If the holomorphic function $f(z)$ is a linear function of the form $a z+b$, then the Weierstrass data $\left(e^{f(z)}, d z\right)$ for $M$ shows that $M$ is an associate surface to the helicoid. Since none of the non-trivial associate surfaces to the helicoid are injective as mappings, then $M$ must be the helicoid itself when $f(z)$ is linear. Thus, it remains to show that $f(z)$ is linear. The formula (77) in Section 2.2 for the Gaussian curvature $K$ and an application of Picard's Theorem imply that $f(z)$ is linear if and only if $M$ has bounded curvature. This fact completes the proof of the theorem in the special case that $K$ is bounded. However, Theorem 4.2 and a clever blow-up argument on the scale of curvature reduces the proof that $f(z)$ is linear in the general case to the case where $K$ is bounded, and so $M$ is a helicoid. For further details, see [136].

Remark 8.1. The first two main steps in the proof outlined above (the second and third paragraphs) can be simplified by using the fact that any embedded minimal multigraph with a large number of sheets contains a submultigraph which can be approximated by the multigraph of a helicoid with an additional logarithmic term, an approximation result which appears as Corollary 14.3 in Colding and Minicozzi [30. Bernstein and Breiner [5], as well as Meeks and Pérez [115, verified that for the double multigraph appearing in the above-sketched proof, this approximation property, together with estimates by Colding and Minicozzi for the separation of the sheets of the multigraph, is sufficient to prove that the surface $M$ intersects each horizontal plane transversely in a single proper arc, which as we observed above, is a delicate step in the proof of Theorem [1.1. An important detail is that

${ }^{38}$ We will discuss this finiteness property in more detail in Section 11 see Conjecture 11.4 
this simplification does not need the hypothesis of simply connectedness and works in the case of proper minimal annuli with one compact boundary curve and infinite total curvature, giving that such an annulus intersects transversely some horizontal plane in a single proper arc. For details, see [4, 5, 115.

Theorem 1.1 solves a long-standing conjecture about the uniqueness of the helicoid among properly embedded, simply connected minimal surfaces in $\mathbb{R}^{3}$. Very little is known about genus- $g$ helicoids with $g \geq 1$. We have already mentioned Theorem 1.4 on the asymptotic behavior and conformal structure of every such minimal surface. It is conjectured that the genus-one helicoid of Hoffman, Karcher and Wei 75, 76, discussed in Section 2.5, is the unique complete, embedded minimal example with this topology. Also, Hoffman and White 85] have given a new approach to the proof of the existence of the genus-one helicoid, which holds the promise of proving the existence of a genus- $g$ helicoid for any $g \in \mathbb{N}$. It is expected (Conjecture 11.6) that for every non-negative integer $g$, there exists a unique nonplanar, properly embedded minimal surface in $\mathbb{R}^{3}$ with genus $g$ and one end.

\section{Minimal Laminations Revisited AND THE EMBEDded CALABI-YAU PROBlem}

In the above sections we have seen that minimal laminations constitute a key tool in the understanding of the global behavior of embedded minimal surfaces in $\mathbb{R}^{3}$. In this section, we will present some results about the existence and structure of minimal laminations, which have deep consequences in various aspects of minimal surface theory, including the general three-manifold setting. For example, we will give a natural condition under which the closure of a complete, embedded minimal surface in a Riemannian three-manifold has the structure of a minimal lamination. From this analysis, we will deduce, among other things, that certain complete, embedded minimal surfaces are proper, a result which is used to prove the uniqueness of the helicoid in the complete setting (Theorem 1.1) by reducing it to the corresponding characterization assuming properness.

9.1. Uniqueness of the helicoid II: The complete case. In the introduction we mentioned the result, proved in 136 by Meeks and Rosenberg, that the closure of a complete, embedded minimal surface $M$ with locally bounded Gaussian curvature 39 in a Riemannian three-manifold $N$, has the structure of a minimal lamination. The same authors have demonstrated that this result still holds true if we substitute the locally bounded curvature assumption by a weaker hypothesis, namely that the injectivity radius of $M$ is positive 40

Definition 9.1. Let $\Sigma$ be a complete Riemannian manifold. The injectivity radius function $I_{\Sigma}: \Sigma \rightarrow(0, \infty]$ is defined at a point $p \in \Sigma$ to be the supremum of the radii of disks centered at the origin in $T_{p} \Sigma$, such that the exponential map at $p$, restricted to each of these disks, is a diffeomorphism onto its image. The injectivity radius of $\Sigma$ is the infimum of $I_{\Sigma}$.

\footnotetext{
${ }^{39}$ This means that for every $p \in N$, there exists a neighborhood of $p$ in $N$ where $M$ has bounded Gaussian curvature.

${ }^{40}$ Actually, the hypothesis is that for every $p \in N$, there exists a neighborhood $D_{p}$ of $p$ in $N$ where the injectivity radius function $I_{M}$ restricted to $M \cap D_{p}$ is bounded away from zero.
} 
If $M$ is an immersed surface in a three-manifold $N$, then $p \in N$ is a limit point of $M$ if either $p \in \bar{M}-M$ or $p \in M$ and there is a sequence $\left\{p_{n}\right\}_{n} \subset M$ which converges to $p$ in $N$ but does not converge to $p$ in the intrinsic topology on $M$. We let $L(M)$ denote the (closed) set of limit points of $M$ in $N$.

The next theorem was proved by Meeks and Rosenberg [137.

Theorem 9.2 (Minimal Lamination Closure Theorem). Let $M$ be a complete, embedded minimal surface of positive injectivity radius in a Riemannian three-manifold $N$ (not necessarily complete). Then, the closure $\bar{M}$ of $M$ in $N$ has the structure of a $C^{1, \alpha}$-minimal lamination $\mathcal{L}$, some of whose leaves are the connected components of $M$.

Furthermore:

1. If $N$ is homogeneously regular, then there exist positive constants $C$ and $\varepsilon$ depending on $N$ and on the injectivity radius of $M$, such that the absolute Gaussian curvature of $M$ in the $\varepsilon$-neighborhood of any limit leaf of $\bar{M}$ is less than $C$.

2. If $M$ is connected, then exactly one of the following three statements holds:

(a) $M$ is properly embedded in $N$ (hence, $L(M)=\varnothing$ ).

(b) The limit set $L(M) \subset \mathcal{L}$ is non-empty and disjoint from $M$, and $M$ is properly embedded in the open set $N-L(M)$.

(c) $L(M)=\mathcal{L}$ and $\mathcal{L}$ contains an uncountable number of leaves.

In the particular case $N=\mathbb{R}^{3}$, more can be said. Suppose $M \subset \mathbb{R}^{3}$ is a connected, complete, embedded minimal surface with positive injectivity radius. By Theorem 9.2 the closure of $M$ has the structure of a minimal lamination of $\mathbb{R}^{3}$. If item 2(a) in Theorem 9.2 does not hold for $M$, then the sublamination of limit points $L(M) \subset \bar{M}$ contains some leaf $L$. By Theorem 4.5, $L$ is stable; hence $L$ is a plane by Theorem 2.25 . Now Theorem 9.2 insures that $M$ has bounded curvature in some $\varepsilon$-neighborhood of the plane $L$, which contradicts Lemma 5.6. This contradiction proves the following result.

Corollary 9.3 (Meeks, Rosenberg 137). Every connected, complete, embedded minimal surface in $\mathbb{R}^{3}$ with positive injectivity radius is properly embedded.

Remark 9.4. Corollary 9.3 is no longer true if we replace $\mathbb{R}^{3}$ by $\mathbb{H}^{3}$ given that Coskunuzer [40] has constructed a complete embedded minimal plane in $\mathbb{H}^{3}$ which is not proper; see especially the last paragraph in 40 for a discussion of the role of this example in $\mathbb{H}^{3}$ in relation to Theorem 9.2 above.

Suppose $M$ is a complete, embedded minimal surface of finite topology in $\mathbb{R}^{3}$. If the injectivity radius of $M$ is zero, then there exists a divergent sequence of embedded geodesic loops $\gamma_{n} \subset M$ (i.e., closed geodesics with at most one corner) with lengths going to zero. Since $M$ has finite topology, we may assume the $\gamma_{n}$ are all contained in a fixed annular end $E$ of $M$. By the Gauss-Bonnet formula, each $\gamma_{n}$ is homotopically non-trivial, and so, the cycles $\gamma_{n} \cup \gamma_{1}, n \geq 2$, bound compact annular subdomains in $E$, whose union is a subend of $E$. However, the GaussBonnet formula implies that the total curvature of this union is finite (greater than $-4 \pi)$. Hence, $E$ is asymptotic to an end of a plane or of a half-catenoid, which is absurd. This argument proves that the following result holds.

Corollary 9.5 (Colding-Minicozzi [37]). A connected, complete, embedded minimal surface of finite topology in $\mathbb{R}^{3}$ is properly embedded. 
The above corollary fails to hold if the surface is allowed to have self-intersections, since there exists a complete minimal disk contained in the unit ball (Nadirashvili [152]). Colding and Minicozzi obtained Corollary 9.5 from a deep analysis of the relation between intrinsic and extrinsic distances for an embedded minimal disk in $\mathbb{R}^{3}$ (see [37]), and the proof of Theorem 9.2 is inspired by this previous work of Colding and Minicozzi.

To finish this section, we remark that one of the central results in this survey article, Theorem 1.1, follows directly from its validity in the proper case and from Corollary 9.5

9.2. The regularity of the singular sets of convergence of minimal laminations. In Sections 2.5 and 4.1 we mentioned a regularity result for the singular set of convergence of a Colding-Minicozzi limit minimal lamination. We next state this result.

Theorem $9.6\left(C^{1,1}\right.$-regularity of $S(\mathcal{L})$, Meeks [11], [112]). Suppose $\left\{M_{n}\right\}_{n}$ is a locally simply connected sequence of properly embedded minimal surfaces in a threemanifold $N$ that converges $C^{\alpha}, \alpha \in(0,1)$, to a minimal lamination $\mathcal{L}$ of $N$, outside a locally finite collection of Lipschitz curves $S(\mathcal{L}) \subset N$ transverse to $\mathcal{L}$ (along which the convergence fails to be $C^{\alpha}$ ). Then, $\mathcal{L}$ is a $C^{1,1}$-minimal foliation in a neighborhood of $S(\mathcal{L})$, and $S(\mathcal{L})$ consists of $C^{1,1}$-curves orthogonal to the leaves of $\mathcal{L}$.

To give an idea of the proof of Theorem 9.6. first note that the nature of this theorem is purely local, so it suffices to consider the case of a sequence of properly embedded minimal disks $M_{n}$ in the unit ball $\mathbb{B}(1)=\mathbb{B}(\overrightarrow{0}, 1)$ of $\mathbb{R}^{3}$ (the general case follows from adapting the arguments to a three-manifold). After passing to a subsequence, one can also assume that the surfaces $M_{n}$ converge to a $C^{0,1}$-minimal foliation $41 \mathcal{L}$ of $\mathbb{B}(1)$ and the convergence is $C^{\alpha}, \alpha \in(0,1)$, outside of a transverse Lipschitz curve $S(\mathcal{L})$ that passes through the origin. Since the unit normal vector field $N_{\mathcal{L}}$ to $\mathcal{L}$ is Lipschitz (Solomon [191]), then the integral curves of $N_{\mathcal{L}}$ are of class $C^{1,1}$. Then the proof consists of demonstrating that $S(\mathcal{L})$ is the integral curve of $N_{\mathcal{L}}$ passing through the origin. To do this, one first proves that $S(\mathcal{L})$ is of class $C^{1}$; hence it admits a continuous tangent field $T$, and then one shows that $T$ is orthogonal to the leaves of $\mathcal{L}$. Crucial in this proof is the uniqueness of the helicoid (Theorem 1.4), since it gives the local picture of the minimal disks $M_{n}$ near the origin as being closely approximated by portions of a highly sheeted helicoid near its axis.

\section{EMBEDDED Minimal SURFACES OF Finite GENUS}

10.1. The Hoffman-Meeks conjecture. By Theorem 1.5, every complete, embedded minimal surface in $\mathbb{R}^{3}$ with finite topology is proper, and hence, orientable. Until the early eighties of the last century, no complete embedded minimal surfaces of finite topology other than the plane, the helicoid (both with genus zero, one end) and the catenoid (genus zero, two ends) were known. For a long time, some geometers supported the conjecture that no other examples of finite topology would exist. The discovery in 1982 of a new genus-one, three-ended embedded example (Costa 42, Hoffman and Meeks [78]) not only disproved this conjecture, but also

\footnotetext{
${ }^{41}$ Any codimension-one minimal foliation is of class $C^{0,1}$ and its unit normal vector field is $C^{0,1}$ as well; see Solomon 191 .
} 
revitalized enormously the interest of geometers in classical minimal surface theory. Since then, a number of new embedded examples have appeared, sometimes even coming in multiparameter families [72, 74, 79, 90, 197, 205].

For complete embedded minimal surfaces with finite topology, there is an interesting dichotomy between the one-end case and those surfaces with more than one end: surfaces in this last case always have finite total curvature (Collin's Theorem 1.3). Only the simplest finite topologies with more than one end have been characterized: the unique examples with genus zero and finite topology are the plane and the catenoid (López and Ros's Theorem 3.2), the catenoid is the unique example with finite genus and two ends (Schoen's Theorem 3.1), and Costa [43, 44] showed that the examples with genus one and three ends lie inside the one-parameter family of surfaces $\left\{M_{1, a} \mid 0<a<\infty\right\}$ that appears in Section 2.5. Today we know many more examples of more complicated finite topologies and more than one end, and up to this date all known examples support the following conjecture.

Conjecture 10.1 (Finite Topology Conjecture, Hoffman, Meeks). A connected surface of finite topology, genus $g$ and $r$ ends, $r>2$, can be properly minimally embedded in $\mathbb{R}^{3}$ if and only if $r \leq g+2$.

Meeks, Pérez and Ros [119] proved the existence of an upper bound for the number of ends of a minimal surface as in the above conjecture solely in terms of the genus; see Theorem 1.6 stated in the Introduction. We next give a sketch of the proof of this result. The argument is by contradiction. The failure of Theorem 1.6 to hold would produce an infinite sequence $\left\{M_{n}\right\}_{n}$ of complete, embedded minimal surfaces in $\mathbb{R}^{3}$ with fixed finite genus $g$ and a strictly increasing number of ends, and which are properly embedded in $\mathbb{R}^{3}$ since they have finite total curvature. Then one analyzes the non-simply connected limits of subsequences of $\left\{M_{n}\right\}_{n}$. The key idea used to achieve these limits is to normalize $M_{n}$ by a translation followed by a homothety on the scale of topology. What we mean here is that we assume that each renormalized surface intersects the closed unit ball of $\mathbb{R}^{3}$ centered at the origin in some non-simply connected component, but that every open ball of radius 1 intersects the surface in simply connected components.

Using the uniformly locally simply connected property of the renormalized sequence $\left\{M_{n}\right\}_{n}$ and the fact that the $M_{n}$ have genus $g$, we analyze what are the possible limits of sequences of $\left\{M_{n}\right\}_{n}$; these limits are either properly embedded, non-simply connected minimal surfaces with genus at most $g$ and possibly infinitely many ends, or parking garage structures on $\mathbb{R}^{3}$ with exactly two columns. The limits which are surfaces with infinitely many ends are then discarded by an application of either a descriptive theorem for the geometry of any properly embedded minimal surface in $\mathbb{R}^{3}$ with finite genus and two limit ends (Meeks, Pérez and Ros [124]), or a non-existence theorem for properly embedded minimal surfaces in $\mathbb{R}^{3}$ with finite genus and one limit end (Meeks, Pérez and Ros [127] or Theorem 10.3 below). The parking garage structure limits are also discarded, by a modification of the argument that eliminates the two-limit-ended limits. Hence, any possible limit $M_{\infty}$ of a subsequence of $\left\{M_{n}\right\}_{n}$ must be a non-simply connected minimal surface, which either has finite total curvature or is a helicoid with positive genus at most $g$. Then, a surgery argument allows one to modify the surfaces $M_{n}$ by replacing compact pieces of $M_{n}$ close to the limit $M_{\infty}$ by a finite number of disks, obtaining a new surface $\widetilde{M}_{n}$ with strictly simpler topology than $M_{n}$ and which is not minimal in the replaced part. A careful study of the replaced parts during the 
sequence allows one to iterate the process of finding non-simply connected minimal limits of the modified surfaces $\widetilde{M}_{n}$. The fact that all the $M_{n}$ have the same finite genus allows one to arrive at a stage in the process of producing limits from which all subsequent limits have genus zero, and so they are catenoids. From this point in the proof, one shows that there exists a large integer $n$ such that $M_{n}$ contains a non-compact planar domain $\Omega \subset M_{n}$ whose boundary consists of two convex planar curves $\Gamma_{1}, \Gamma_{2}$ in parallel planes, such that each $\Gamma_{i}$ separates $M_{n}$ and has flux orthogonal to the plane that contains $\Gamma_{i}$. In this setting, the López-Ros deformation defined in Section 2.2 (see [104, 171]) applies to $\Omega$, giving the desired contradiction. This finishes the sketch of the proof of Theorem 1.6.

Recall that the Jorge-Meeks formula (equation (9) in Section 2.3) relates the degree of the Gauss map of a complete, embedded minimal surface with finite total curvature in $\mathbb{R}^{3}$, with its genus and number of ends. Since the finite index of stability of a complete minimal surface of finite total curvature can be estimated from above in terms of the degree of its Gauss map (Tysk 203]), Theorem 1.6 has the following important theoretical consequence.

Corollary 10.2 (Meeks, Pérez, Ros [119]). For every $g \in \mathbb{N} \cup\{0\}$, there exists an integer $i(g)$ such that if $M \subset \mathbb{R}^{3}$ is a complete, embedded minimal surface with genus $g$ and a finite number of ends greater than 1 , then the index of stability of $M$ is at most $i(g)$.

The above paragraphs deal with complete, embedded minimal surfaces of finite topology and more than one end. The case of one-ended surfaces of finite genus was covered by Theorems 1.1 and 1.4 see also the discussion about genus- $k$ helicoids in Section 2.5 and Conjecture 11.6 below.

10.2. Non-existence of examples with one limit end and finite genus. Next we consider properly embedded minimal surfaces with finite genus and infinite topology. Note that we have included the hypothesis "proper" instead of "complete" since we can no longer apply Theorem 1.5 in this setting 42 Since the number of ends of such a surface $M \subset \mathbb{R}^{3}$ is infinite and the set of ends $\mathcal{E}(M)$ of $M$ is compact (see Section 2.7), then $M$ must have at least one limit end. Up to a rotation, we can assume that the limit tangent plane at infinity of $M$ (Section [) is horizontal. Theorem 7.9 insures that $M$ has no middle limit ends; hence either it has one limit end (this one being the top or the bottom limit end) or both top and bottom ends are the limit ends of $M$, as in a Riemann minimal example. Meeks, Pérez and Ros [127] have discarded the one limit end case through the following result.

Theorem 10.3 (Meeks, Pérez, Ros [127]). If $M \subset \mathbb{R}^{3}$ is a properly embedded minimal surface with finite genus 43 then $M$ cannot have exactly one limit end. Furthermore, $M$ is recurrent.

Sketch of the proof. Assume $M$ is a properly embedded minimal surface in $\mathbb{R}^{3}$ with finite genus and exactly one limit end. After a rotation, we can suppose that $M$ has horizontal limit tangent plane at infinity and its set of ends, linearly ordered by

\footnotetext{
${ }^{42}$ However, a result of Meeks, Pérez and Ros [120] states that a connected, complete, embedded minimal surface of finite genus in $\mathbb{R}^{3}$ which has at most a countable number of limit ends is always properly embedded.

43 The hypothesis on finite genus is essential here, since there exist properly embedded minimal surfaces in $\mathbb{R}^{3}$ with infinite genus and just one limit end (Traizet [200]).
} 
increasing heights (see the Ordering Theorem6.2), is $\mathcal{E}(M)=\left\{e_{1}, e_{2}, \ldots, e_{\infty}\right\}$ with the limit end of $M$ being its top end $e_{\infty}$. One first needs a previous description of the asymptotic behavior of $M$ by Meeks, Pérez and Ros (Theorem 2 in [126]): each non-limit end $e_{n} \in \mathcal{E}(M)$ is asymptotic to a graphical annular end $E_{n}$ of a vertical catenoid with negative logarithmic growth $a_{n}$ satisfying $a_{1} \leq \cdots \leq a_{n} \leq$ $\cdots<0$. The next step consists of a detailed analysis of the limits (after passing to a subsequence) of homothetic shrinkings $\left\{\lambda_{n} M\right\}_{n}$, where $\left\{\lambda_{n}\right\}_{n} \subset \mathbb{R}^{+}$is any sequence of numbers decaying to zero; one first shows that $\left\{\lambda_{n} M\right\}_{n}$ is locally simply connected in $\mathbb{R}^{3}-\{\overrightarrow{0}\}$. This is a difficult technical part of the proof, where the results of Colding and Minicozzi again play a crucial role. Then one proves that the limits of subsequences of $\left\{\lambda_{n} M\right\}_{n}$ consist of (possibly singular) minimal laminations $\mathcal{L}$ of $H(*)=\left\{x_{3} \geq 0\right\}-\{\overrightarrow{0}\} \subset \mathbb{R}^{3}$ containing $\partial H(*)$ as a leaf. Subsequently, one checks that every such limit lamination $\mathcal{L}$ is smooth and that the singular set of convergence $S(\mathcal{L})$ of $\lambda_{n} M$ to $\mathcal{L}$ is empty (we will discuss this step a bit more after this sketched proof). In particular, taking $\lambda_{n}=\left\|p_{n}\right\|^{-1}$, where $p_{n}$ is any divergent sequence on $M$, the fact that $S(\mathcal{L})=\varnothing$ for the corresponding limit minimal lamination $\mathcal{L}$ insures that the Gaussian curvature of $M$ decays at least quadratically in terms of the distance function to the origin. Meeks, Pérez and Ros 122 proved that if an embedded minimal surface has quadratic decay of curvature, then it has finite total curvature. This is impossible in our situation with infinitely many ends and finishes the outline of the proof of the first statement of Theorem 10.3 .

In order to finish the sketch of the proof, it only remains to check that $M$ is recurrent. If $M$ has exactly one end, then $M$ is conformally a compact Riemann surface minus one point (Theorem 1.4) and so, $M$ is recurrent. If $M$ has a finite number of ends greater than one, then $M$ has finite total curvature (Theorem 1.3). By the Huber-Osserman Theorem 2.11, $M$ is conformally a compact Riemann surface minus a finite number of points; thus it is again recurrent. Finally, if $M$ has infinitely many ends, then $M$ has exactly two limit ends; see the paragraph just before the statement of Theorem 10.3. In this situation, Theorem 7.10 asserts that $M$ is recurrent, thereby finishing our sketch of the proof.

There is a crucial point in the above sketch of the proof of Theorem 10.3, which should be emphasized. We mentioned above that any limit lamination $\mathcal{L}$ of $H(*)$ obtained as a limit of (a subsequence of) homothetic shrinkings $\left\{\lambda_{n} M\right\}_{n}$ with $\lambda_{n} \searrow 0$, has no singularities and empty singular set of convergence $S(\mathcal{L})$. These properties are consequences of what we call the Stability Lemma (see Lemma 10.5), which we want to explain now. If such a lamination $\mathcal{L}$ had singularities or if $S(\mathcal{L})$ were non-empty for a given sequence of shrinkings of $M$, then we would find some smooth limit leaf $L$ of $\mathcal{L}$, and hence $L$ would be stable. The difficulty in discarding this possibility lies in the fact that the stable leaves of $\mathcal{L}$, while perhaps proper in $\left\{x_{3}>0\right\}$, may not be complete and so, we do not know they must be planes. It is not difficult to prove that the smooth stable leaves in $\mathcal{L}$ in fact satisfy the hypotheses of the Stability Lemma 10.5 below, and so they are planes. Once one has that the smooth stable leaves in $\mathcal{L}$ are planes, then the proof of Theorem 4.2 leads to a contradiction, thereby showing that $\mathcal{L}$ has no singularities and $S(\mathcal{L})$ is empty.

We include below the proof of the Stability Lemma for three reasons: 
(1) To illustrate how one can obtain information on the conformal structure of possibly incomplete minimal surfaces by studying conformally related metrics, and then how to apply such information to constrain their geometry.

(2) Because Theorem 2.25 is a direct consequence of Lemma 10.5.

(3) Because the short proof of Lemma10.5 below indicates some new techniques and insights for possibly solving the following famous conjecture, due to Gulliver and Lawson (also note that the following conjecture is a special case of the Fundamental Singularity Conjecture 11.3).

Conjecture 10.4 (Isolated Singularity Conjecture). The closure of a properly embedded minimal surface in the punctured closed unit ball $\overline{\mathbb{B}}(1)-\{\overrightarrow{0}\}$ is a compact embedded minimal surface.

The next lemma by Meeks, Pérez, Ros 122 was also found independently by Colding and Minicozzi 24, and it is motivated by a similar result of Meeks, Pérez and Ros in [127.

Lemma 10.5 (Stability Lemma). Let $L \subset \mathbb{R}^{3}-\{\overrightarrow{0}\}$ be a stable, orientable, minimal surface which is complete outside the origin. Then its closure $\bar{L}$ is a plane.

Proof. Consider the metric $\widetilde{g}=\frac{1}{R^{2}} g$ on $L$, where $g$ is the metric induced by the usual inner product $\langle$,$\rangle of \mathbb{R}^{3}$. Note that if $L$ were a plane through $\overrightarrow{0}$, then $\widetilde{g}$ would be the metric on $L$ of an infinite cylinder of radius 1 with ends at $\overrightarrow{0}$ and at infinity. We will show that, in general, this metric is complete on $L$ and that the assumption of stability can be used to show that $(L, g)$ is flat. Since $\left(\mathbb{R}^{3}-\{\overrightarrow{0}\}, \widehat{g}\right)$ with $\widehat{g}=\frac{1}{R^{2}}\langle$, is isometric to $\mathbb{S}^{2} \times \mathbb{R}$ and $L$ is complete outside $\overrightarrow{0}$, then $(L, \widetilde{g}) \subset\left(\mathbb{R}^{3}-\{\overrightarrow{0}\}, \widehat{g}\right)$ is complete.

We now prove that $(L, g)$ is flat. The Laplacians and Gauss curvatures of $g, \widetilde{g}$ are related by the equations $\widetilde{\Delta}=R^{2} \Delta$ and $\widetilde{K}=R^{2}\left(K_{L}+\Delta \log R\right)$. Since $\Delta \log R=$ $\frac{2\left(1-\|\nabla R\|^{2}\right)}{R^{2}} \geq 0$, then

$$
-\widetilde{\Delta}+\widetilde{K}=R^{2}\left(-\Delta+K_{L}+\Delta \log R\right) \geq R^{2}\left(-\Delta+K_{L}\right) .
$$

Since $K_{L} \leq 0$ and $(L, g)$ is stable, $-\Delta+K_{L} \geq-\Delta+2 K_{L} \geq 0$, and so, $-\widetilde{\Delta}+\widetilde{K} \geq 0$ on $(L, \widetilde{g})$. As $\widetilde{g}$ is complete, the universal covering of $L$ is conformally $\mathbb{C}$ (FischerColbrie and Schoen 60]). Since $(L, g)$ is stable, there exists a positive Jacobi function $u$ on $L$ (Fischer-Colbrie [59]). Passing to the universal covering $\widehat{L}, \Delta \widehat{u}=$ $2 K_{\widehat{L}} \widehat{u} \leq 0$, and so, $\widehat{u}$ is a positive superharmonic on $\mathbb{C}$, and hence constant. Thus, $0=\Delta u-2 K_{L} u=-2 K_{L} u$ on $L$, which means that $K_{L}=0$.

10.3. Uniqueness of the Riemann minimal examples. If a properly embedded minimal surface $M \subset \mathbb{R}^{3}$ has finite genus and infinite topology, then Theorems 7.9 and 10.3 imply that $M$ has two limit ends which are its top and bottom ends (after a rotation so that the limit tangent plane at infinity of $M$ is horizontal). The classical model in this setting is any of the surfaces in the one-parameter family of Riemann minimal examples; see Section 2.5. Meeks, Pérez and Ros [124 have characterized these last surfaces as the unique properly embedded minimal surfaces in $\mathbb{R}^{3}$ with genus zero and infinite topology (Theorem 10.6 below), a uniqueness result that, together with Theorems $1.3,1.4$ and 3.2 finishes the classification of all properly embedded minimal planar domains in $\mathbb{R}^{3}$ stated in Theorem 1.2 We will explain the main steps in the proof of this characterization below; see also [118] for further details. 
Theorem 10.6. Every properly embedded, minimal planar domain $M \subset \mathbb{R}^{3}$ with infinite topology is a Riemann minimal example.

Sketch of the proof. We first rotate $M$ in $\mathbb{R}^{3}$ to have horizontal limit tangent plane at infinity. Next, we rescale $M$ by a suitable homothety so that the vertical component of the flux vector of $M$ along a compact horizontal section $M \cap\left\{x_{3}=\right.$ constant $\}$ is equal to 1. At this point we need a previous analytic and geometric description of $M$ (Theorem 1 in Meeks, Pérez and Ros [126]):

(A) $M$ can be conformally parametrized by the cylinder $\mathbb{C} /\langle i\rangle$ (here $i=\sqrt{-1}$ ) punctured in an infinite discrete set of points $\left\{p_{j}, q_{j}\right\}_{j \in \mathbb{Z}}$ which correspond to the planar ends of $M$.

(B) The stereographically projected Gauss map $g:(\mathbb{C} /\langle i\rangle)-\left\{p_{j}, q_{j}\right\}_{j \in \mathbb{Z}} \rightarrow \mathbb{C} \cup$ $\{\infty\}$ extends through the planar ends of $M$ to a meromorphic function $g$ on $\mathbb{C} /\langle i\rangle$ which has double zeros at the points $p_{j}$ and double poles at the $q_{j}$

(C) The height differential of $M$ is $d h=d z$ with $z$ being the usual conformal coordinate on $\mathbb{C}$; hence the third coordinate function of $M$ is $x_{3}(z)=\Re(z)$.

(D) The planar ends of $M$ are ordered by their heights so that $\Re\left(p_{j}\right)<\Re\left(q_{j}\right)<$ $\Re\left(p_{j+1}\right)$ for all $j$ with $\Re\left(p_{j}\right) \rightarrow \infty$ (resp. $\left.\Re\left(p_{j}\right) \rightarrow-\infty\right)$ when $j \rightarrow \infty$ (resp. $j \rightarrow-\infty)$.

(E) The flux vector $F$ of $M$ along a compact horizontal section has non-zero horizontal component; hence after a suitable rotation around the $x_{3}$-axis, $F=(h, 0,1)$ for some $h>0$.

(F) With the normalizations above, the Gaussian curvature of $M$ is bounded and the vertical spacings between consecutive planar ends are bounded from above and below by positive constants, with all these constants depending only on $h$. This is a non-trivial application of Colding-Minicozzi theory [126].

(G) For every divergent sequence $\left\{z_{k}\right\}_{k} \subset \mathbb{C} /\langle i\rangle$, there exists a subsequence of the meromorphic functions $g_{k}(z)=g\left(z+z_{k}\right)$ which converges uniformly on compact subsets of $\mathbb{C} /\langle i\rangle$ to a non-constant meromorphic function $g_{\infty}: \mathbb{C} /\langle i\rangle \rightarrow \mathbb{C} \cup\{\infty\}$ (we will refer to this property by saying that $g$ is quasiperiodic). In fact, $g_{\infty}$ corresponds to the Gauss map of a minimal surface $M_{\infty}$ satisfying the same properties as $M$, which is the limit of a related subsequence of translations of $M$ by vectors whose $x_{3}$-components are $\Re\left(z_{k}\right)$. Such a limit exists by an application of item (F) above.

Let $\mathcal{M}$ be the space of properly embedded, minimal planar domains $M \subset \mathbb{R}^{3}$ with two limit ends and flux $F=(h, 0,1)$ for some $h=h(M)>0$, identified up to translations. Every minimal surface $M \in \mathcal{M}$ admits a classical Jacobi function called its Shiffman function $S_{M}$, which measures the curvature variation of the parallel sections of $M$. Analytically, $S_{M}$ is given by

$$
S_{M}=\Lambda \frac{\partial \kappa_{c}}{\partial y}=\Im\left[\frac{3}{2}\left(\frac{g^{\prime}}{g}\right)^{2}-\frac{g^{\prime \prime}}{g}-\frac{1}{1+|g|^{2}}\left(\frac{g^{\prime}}{g}\right)^{2}\right],
$$

where $\Lambda$ is the conformal factor between the induced metric on $M$ by the inner product of $\mathbb{R}^{3}$ and the flat metric in the conformal coordinate $z=x+i y$, i.e., $d s^{2}=\Lambda^{2}|d z|^{2}$, and $\kappa_{c}(y)$ is the planar curvature of the level curve $M \cap\left\{x_{3}=c\right\}$ $(c \in \mathbb{R})$, which is parameterized by $z_{c}(y)=c+i y$ since $x_{3}(z)=\Re(z)=x$. The 
second equality in (13) expresses $S_{M}$ solely as a rational expression of $g$ and its derivatives with respect to $z$ up to order 2 .

Since $\Lambda$ is a positive function, the zeros of $S_{M}$ coincide with the critical points of $\kappa_{c}(y)$. Thus, $S_{M}$ vanishes identically if and only if $M$ is foliated by circles and straight lines in horizontal planes. In a posthumously published paper, B. Riemann [175, 176] classified all minimal surfaces with such a foliation property: they reduce to the plane, catenoid, helicoid and the 1-parameter family of Riemann minimal examples (which took this name from this discovery of Riemann). Therefore, a possible approach to proving Theorem 10.6 is to verify that $S_{M}=0$, but instead of proving this fact directly, Meeks, Pérez and Ros demonstrated that $S_{M}$ can be integrated in the following sense:

Given a minimal surface $M \in \mathcal{M}$, there exists a one-parameter family $\left\{M_{t}\right\}_{t} \subset \mathcal{M}$ such that $M_{0}=M$ and the normal variational vector field for this variation, when restricted to each $M_{t}$, is the Shiffman Jacobi function $S_{M_{t}}$ multiplied by the unit normal vector field to $M_{t}$.

In fact, the parameter $t$ of this deformation can be extended to be a complex number, and $t \mapsto M_{t}$ can be viewed as the real part of a complex-valued holomorphic curve in a certain complex variety. This is a very special integration property for $S_{M}$, which we refer to by saying that the Shiffman function can be holomorphically integrated for every surface $M \in \mathcal{M}$.

The method for proving that the Shiffman function $S_{M}$ can be holomorphically integrated for every such $M$ is through the Korteweg-de Vries equation (KdV) and its hierarchy, two fundamental tools in integrable systems theory. We recommend that the interested reader consult the excellent survey by Gesztesy and Weikard 65] for an overview of the notions and properties which we will use in the sequel. Briefly, the way to obtain the KdV equation from the Shiffman function is as follows.

It turns out that $S_{M}$ admits a globally defined conjugate Jacobi function $44 S_{M}^{*}$ on $(\mathbb{C} /\langle i\rangle)-B(N)$, where $B(N)$ denotes the set of branch points of the Gauss map $N$ of $M$. Thus, $S_{M}+i S_{M}^{*}$ can be viewed as the support function of a holomorphic map from $(\mathbb{C} /\langle i\rangle)-B(N)$ into $\mathbb{C}^{3}$, namely $S_{M}+i S_{M}^{*}=\left\langle X_{u}+i\left(X_{u}\right)^{*}, N\right\rangle$. The holomorphicity of $X_{u}+i\left(X_{u}\right)^{*}$ allows us to identify $S_{M}+i S_{M}^{*}$ with an infinitesimal deformation in the space $\mathcal{W}$ of quasiperiodic meromorphic functions on $\mathbb{C} /\langle i\rangle$ with double zeros and double poles, i.e., with the derivative $\dot{g}_{S}=\left.\frac{d}{d t}\right|_{t=0} g_{t}$ of a holomorphic curve $t \in \mathbb{D}(\varepsilon)=\{t \in \mathbb{C}|| t \mid<\varepsilon\} \mapsto g_{t} \in \mathcal{W}$ with $g_{0}=g$, which can be computed from (13) as

$$
\dot{g}_{S}=\frac{i}{2}\left(g^{\prime \prime \prime}-3 \frac{g^{\prime} g^{\prime \prime}}{g}+\frac{3}{2} \frac{\left(g^{\prime}\right)^{3}}{g^{2}}\right) .
$$

Therefore, to integrate $S_{M}$ holomorphically one needs to find a holomorphic curve $t \in \mathbb{D}(\varepsilon) \mapsto g_{t} \in \mathcal{W}$ with $g_{0}=g$, such that for all $t$ the pair $\left(g_{t}, d z\right)$ is the Weierstrass data of a minimal surface $M_{t} \in \mathcal{M}$ and $\left.\frac{d}{d t}\right|_{t} g_{t}=\frac{i}{2}\left(g_{t}^{\prime \prime \prime}-3 \frac{g_{t}^{\prime} g_{t}^{\prime \prime}}{g_{t}}+\frac{3}{2} \frac{\left(g_{t}^{\prime}\right)^{3}}{g_{t}^{2}}\right)$.

Viewing (14) as an evolution equation (in complex time $t$ ), one could apply general techniques to find solutions $g_{t}=g_{t}(z)$ defined locally around a point $z_{0} \in$

\footnotetext{
${ }^{44}$ The conjugate Jacobi function of a Jacobi function $u$ over a minimal surface $M$ is the (locally defined) support function $u^{*}$ of the conjugate surface $\left(X_{u}\right)^{*}$ of the branched minimal surface $X_{u}$ associated to $u$ by the Montiel-Ros correspondence; see Theorem 2.29 In particular, both $X_{u}$ and $\left(X_{u}\right)^{*}$ have the same Gauss map as $M$, and $u^{*}$ also satisfies the Jacobi equation.
} 
$(\mathbb{C} /\langle i\rangle)-g^{-1}(\{0, \infty\})$ with the initial condition $g_{0}=g$, but such solutions are not necessarily defined on the whole cylinder, can develop essential singularities, and even if they were meromorphic on $\mathbb{C} /\langle i\rangle$, it is not clear a priori that they would have only double zeros and poles and other properties necessary to give rise to minimal surfaces $M_{t}$ in $\mathcal{M}$. All these problems are solved by arguments related to the theory of the (meromorphic) KdV equation, as we will next explain.

The change of variables

$$
u=-\frac{3\left(g^{\prime}\right)^{2}}{4 g^{2}}+\frac{g^{\prime \prime}}{2 g}
$$

transforms (14) into the evolution equation

$$
\frac{\partial u}{\partial t}=-u^{\prime \prime \prime}-6 u u^{\prime}
$$

which is the KdV equation 45 The reader may wonder why the KdV equation appears in connection to the Shiffman function. The change of variables $x=g^{\prime} / g$ transforms the expression (14) for $\dot{g}_{S}$ into the evolution equation $\dot{x}=\frac{i}{2}\left(x^{\prime \prime \prime}-\frac{3}{2} x^{2} x^{\prime}\right)$, called a modified $K d V$ equation $(\mathrm{mKdV})$. It is well known that $\mathrm{mKdV}$ equations in $x$ can be transformed into $\mathrm{KdV}$ equations in $u$ through the so-called Miura transformations, $x \mapsto u=a x^{\prime}+b x^{2}$ with $a, b$ suitable constants; see for example [65], page 273. Equation (15) is nothing but the composition of $g \mapsto x$ and a Miura transformation. The holomorphic integration of the Shiffman function $S_{M}$ could be performed just in terms of the theory of the $\mathrm{mKdV}$ equation, but we will instead use the more standard KdV theory.

Coming back to the holomorphic integration of $S_{M}$, this integration amounts to solving globally in $\mathbb{C} /\langle i\rangle$ the Cauchy problem for equation (16), i.e. finding a meromorphic solution $u(z, t)$ of (16) with $z \in \mathbb{C} /\langle i\rangle$ and $t \in \mathbb{D}(\varepsilon)$, whose initial condition is $u(z, 0)=u(z)$ given by (15). It is a well-known fact in KdV theory (see for instance [65] and also see Segal and Wilson [189]) that such a Cauchy problem can be solved globally producing a holomorphic curve $t \mapsto u_{t}$ of meromorphic functions $u(z, t)=u_{t}(z)$ on $\mathbb{C} /\langle i\rangle$ (with controlled Laurent expansions in poles of $u_{t}$ ) provided that the initial condition $u(z)$ is an algebro-geometric potential for the $\mathrm{KdV}$ equation. To understand this last notion, one must view (16) as the case $n=1$ of a sequence of evolution equations in $u$ called the $K d V$ hierarchy,

$$
\left\{\frac{\partial u}{\partial t_{n}}=-\partial_{z} \mathcal{P}_{n+1}(u)\right\}_{n \geq 0}
$$

where $\mathcal{P}_{n+1}(u)$ is a differential operator given by a polynomial expression of $u$ and its derivatives with respect to $z$ up to order $2 n$. These operators, which are closely related to Lax Pairs (see Section 2.3 in [65) are defined by the recurrence law

$$
\left\{\begin{array}{l}
\partial_{z} \mathcal{P}_{n+1}(u)=\left(\partial_{z z z}+4 u \partial_{z}+2 u^{\prime}\right) \mathcal{P}_{n}(u) \\
\mathcal{P}_{0}(u)=\frac{1}{2}
\end{array}\right.
$$

In particular, $\mathcal{P}_{1}(u)=u$ and $\mathcal{P}_{2}(u)=u^{\prime \prime}+3 u^{2}$ (plugging $\mathcal{P}_{2}(u)$ in (17) one obtains the $\mathrm{KdV}$ equation). Hence, for each $n \in \mathbb{N} \cup\{0\}$ one must consider the right-hand side of the $n$th equation in (17) as a polynomial expression of $u=u(z)$ and its derivatives with respect to $z$ up to order $2 n+1$. We will call this expression a flow,

\footnotetext{
${ }^{45}$ In the literature one can find different normalizations of the KdV equation, given by different coefficients for $u^{\prime \prime \prime}, u u^{\prime}$ in equation (16); all of them are equivalent up to a change of variables.
} 
denoted by $\frac{\partial u}{\partial t_{n}}$. A function $u(z)$ is said to be an algebro-geometric potential of the $\mathrm{KdV}$ equation if there exists a flow $\frac{\partial u}{\partial t_{n}}$ which is a linear combination of the lower order flows in the KdV hierarchy.

With all these ingredients, one needs to check that for every minimal surface $M \in \mathcal{M}$, the function $u=u(z)$ defined by equation (15) in terms of the Gauss map $g(z)$ of $M$, is an algebro-geometric potential of the $\mathrm{KdV}$ equation. This property follows from a combination of the following two facts:

1. Each flow $\frac{\partial u}{\partial t_{n}}$ in the KdV hierarchy produces a bounded, complex-valued Jacobi function $v_{n}$ on $\mathbb{C} /\langle i\rangle$ in a similar manner to the way that $\frac{\partial u}{\partial t_{1}}$ produces the complex Shiffman function $S_{M}+i S_{M}^{*}$.

2. Since the Jacobi functions $v_{n}$ produced in item 1 are bounded on $\mathbb{C} /\langle i\rangle$, they can be considered to lie in the kernel of a Schrödinger operator $L_{M}$ on $\mathbb{C} /\langle i\rangle$ with bounded potential; namely, $L_{M}=\left(\Delta_{\mathbb{S} 1}+\partial_{t}^{2}\right)+V_{M}$, where $\mathbb{C} /\langle i\rangle$ has been isometrically identified with $\mathbb{S}^{1} \times \mathbb{R}$ endowed with the usual product metric $d \theta^{2} \times d t^{2}$, and the potential $V_{M}$ is the square of the norm of the differential of the Gauss map of $M$ with respect to $d \theta^{2} \times d t^{2}\left(V_{M}\right.$ is bounded since $M$ has bounded Gaussian curvature; see property (F) above). Finally, the kernel of $L_{M}$ restricted to bounded functions is finite dimensional; this finite dimensionality was proved by Meeks, Pérez and Ro:46 in 124 and also follows from a more general result by Colding, de Lellis and Minicozzi 23]).

The aforementioned control on the Laurent expansions in poles of $u_{t}$, coming from the integration of the Cauchy problem for the KdV equation, is enough to prove that the corresponding meromorphic function $g_{t}$ associated to $u_{t}$ by (15) has the correct behavior in poles and zeros; this property, together with the fact that both $S_{M}, S_{M}^{*}$ preserve infinitesimally the complex periods along any closed curve in $\mathbb{C} /\langle i\rangle$, suffices to show that the Weierstrass data $\left(g_{t}, d z\right)$ solves the period problem and defines $M_{t} \in \mathcal{M}$ with the desired properties.

Finally, once the holomorphic integrability of $S_{M}$ for any $M \in \mathcal{M}$ by a curve $t \mapsto M_{t} \in \mathcal{W}$ with $M_{0}=M$ is proved, a compactness argument based on the fact that $t \mapsto M_{t}$ preserves the flux along every compact horizontal section gives that $\dot{g}_{S}$ given by (14) vanishes identically, which in turn implies that $S_{M}+i S_{M}^{*}$ is a complex-valued linear function of the Gauss map, $S_{M}+i S_{M}^{*}=\langle a, N\rangle$ for some $a \in \mathbb{C}^{3}$. This property can be used to show that $M$ is invariant by a translation $T$, inducing a properly embedded minimal torus in some quotient $\mathbb{R}^{3} / T$ with total curvature $-8 \pi$. Under these conditions, a previous classification theorem by Meeks, Pérez and Ros 125 leads to the desired property that $M$ is a Riemann minimal example, which finishes the sketch of the proof.

The classification Theorem 10.6 can be used to analyze the asymptotic behavior of properly embedded minimal surfaces with finite genus and infinitely many ends. Roughly speaking, it asserts that if $M$ is a properly embedded minimal surface in $\mathbb{R}^{3}$ with finite genus $g$ and an infinite number of ends, then $M$ has two limit ends and each of its limit ends is asymptotic as $x_{3} \rightarrow \pm \infty$ to one of the limit ends of the Riemann minimal example with horizontal tangent plane at infinity and the same flux vector as $M$ (see Theorem 9.1 in [124] for details).

\footnotetext{
${ }^{46}$ This follows arguments by Pacard (personal communication), which in turn are inspired in a paper by Lockhart and McOwen 102 .
} 


\section{Outstanding PROBlEMs AND CONJECTURES}

In this last section, we present many of the fundamental conjectures in classical minimal surface theory. Hopefully, our presentation and discussion of these problems will speed up their solution and stimulate further interest in this beautiful subject. We have listed in the statement of each conjecture the principal researchers to whom the conjecture might be attributed. We consider all of these problems to be of fundamental importance and we note that they are not listed in order of significance, difficulty or presumed depth.

Some of these problems and others appear in 110, or in 113, along with further discussions. Also see the first author's 1978 book [105, for a long list of conjectures in the subject, some of whose solutions we have discussed in our survey presented here.

Conjecture 11.1 (Convex Curve Conjecture, Meeks). Two convex Jordan curves in parallel planes cannot bound a compact minimal surface of positive genus.

There are some partial results on the Convex Curve Conjecture, under the assumption of some symmetry on the curves (see Meeks and White [141, Ros [178] and Schoen 188]). Also, the results of 141, 142 indicate that the Convex Curve Conjecture probably holds in the more general case where the two convex planar curves do not necessarily lie in parallel planes, but rather lie on the boundary of their convex hull; in this case, the planar Jordan curves are called extremal. Results by Ekholm, White and Wienholtz $[51$ imply that every compact, orientable minimal surface that arises as a counterexample to the Convex Curve Conjecture is embedded. Based on work in [51, Tinaglia [194 proved that for a fixed pair of extremal, convex planar curves, there is a bound on the genus of such a minimal surface. More generally, Meeks [105] has conjectured that if $\Gamma=\left\{\alpha, \beta_{1}, \beta_{2}, . ., \beta_{n}\right\} \subset \mathbb{R}^{3}$ is a finite collection of planar, convex, simple closed curves with $\alpha$ in one plane and such that $\beta_{1}, \beta_{2}, \ldots, \beta_{n}$ bound a pairwise disjoint collection of disks in a parallel plane, then any compact minimal surface with boundary $\Gamma$ must have genus zero.

Conjecture 11.2 ( $4 \pi$-Conjecture, Meeks, Yau, Nitsche). If $\Gamma$ is a simple closed curve in $\mathbb{R}^{3}$ with total curvature at most $4 \pi$, then $\Gamma$ bounds a unique compact, orientable, branched minimal surface and this unique minimal surface is an embedded disk.

As partial results to this conjecture, it is worth mentioning that Nitsche 159. proved that a regular analytic Jordan curve in $\mathbb{R}^{3}$ whose total curvature is at most $4 \pi$ bounds a unique minimal disk; recall also (Theorem 2.32 above) that Meeks and Yau [145] demonstrated the conjecture if $\Gamma$ is a $C^{2}$-extremal curve (they even allowed the minimal surface spanned by $\Gamma$ to be non-orientable). Concerning this weakening of Conjecture 11.2 by removing the orientability assumption on the minimal surface spanning $\Gamma$, we mention the following generalized conjecture due to Ekholm, White and Wienholtz [51]:

Besides the unique minimal disk given by Nitsche's Theorem [159], only one or two Möbius strips can occur, and if the total curvature of $\Gamma$ is at most $3 \pi$, then there are no such Möbius strip examples.

Passing to a different conjecture, Gulliver and Lawson 68 proved that if $\Sigma$ is an orientable, stable minimal surface with compact boundary that is properly embedded in the punctured unit ball $\mathbb{B}-\{\overrightarrow{0}\}$ of $\mathbb{R}^{3}$, then its closure is a compact, embedded 
minimal surface; also see Conjecture 10.4 above. If $\Sigma$ is not stable, then the corresponding result is not known. Nevertheless, Meeks, Pérez and Ros [122, 127] proved that every properly embedded minimal surface $M$ in $\mathbb{B}-\{\overrightarrow{0}\}$ with $\partial M \subset \mathbb{S}^{2}$ extends across the origin provided that $K|R|^{2}$ is bounded on $M$, where $K$ is the Gaussian curvature function of $M$ and $R^{2}=x_{1}^{2}+x_{2}^{2}+x_{3}^{2}$ (Theorem 2.27 implies that this inequality holds if $M$ is stable). In fact, the boundedness of $|K| R^{2}$ is equivalent to the removability of the singularity of $M$ at the origin, and this removable singularity result holds true if we replace $\mathbb{R}^{3}$ by an arbitrary Riemannian three-manifold. Meeks, Pérez and Ros have conjectured that this removable singularity result holds true if we replace the origin by any closed set in $\mathbb{R}^{3}$ with zero one-dimensional Hausdorff measure and the surface is assumed to be properly embedded in the complement of this set; see Conjecture 11.3 below.

The most ambitious conjecture about removable singularities for minimal surfaces is the following one, which deals with lamination instead of with surfaces. Recall that Examples I, II and III in Section 4.2 indicate that one cannot expect the next conjecture to be true if we replace $\mathbb{R}^{3}$ by $\mathbb{H}^{3}, \mathbb{H}^{2} \times \mathbb{R}$ or by a ball in $\mathbb{R}^{3}$.

Conjecture 11.3 (Fundamental Singularity Conjecture, Meeks, Pérez, Ros). If $A \subset \mathbb{R}^{3}$ is a closed set with zero 1-dimensional Hausdorff measure and $\mathcal{L}$ is a minimal lamination of $\mathbb{R}^{3}-A$, then $\mathcal{L}$ extends to a minimal lamination of $\mathbb{R}^{3}$.

In Section 8 we mentioned that a key part of the proof of the uniqueness of the helicoid by Meeks and Rosenberg relies on a finiteness result for the number of components of a minimal graph over a proper domain of $\mathbb{R}^{2}$ with zero boundary values. More precisely, they proved that if $\mathcal{D}$ is a proper, possibly disconnected domain of $\mathbb{R}^{2}$ and $u: \mathcal{D} \rightarrow \mathbb{R}$ is a solution of the minimal surface equation (1D) in $\mathcal{D}$ with zero boundary values and bounded gradient, then $\mathcal{D}$ has at most a finite number of components where $u$ is non-zero. This technical property can be viewed as an important partial result in the direction of the solution of the following conjecture, made by Meeks a number of years earlier.

Conjecture 11.4 (Connected Graph Conjecture, Meeks). A minimal graph in $\mathbb{R}^{3}$ with zero boundary values over a proper, possibly disconnected domain in $\mathbb{R}^{2}$ can have at most two non-planar components. Furthermore, if the graph also has sublinear growth, then such a graph with no planar components is connected.

Consider a proper, possibly disconnected domain $\mathcal{D}$ in $\mathbb{R}^{2}$ and a solution $u: \mathcal{D} \rightarrow$ $\mathbb{R}$ of the minimal surface equation with zero boundary values, such that $u$ is non-zero on each component of $\mathcal{D}$. There are several partial results related to Conjecture11.4. In 1981, Mikklyukov 147] proved that if each component of $\mathcal{D}$ is simply connected with a finite number of boundary components, then $\mathcal{D}$ has at most three components (in fact, with current tools, it can be shown that his method applies to the case that $\mathcal{D}$ has finitely generated first homology group [148]). Earlier, Nitsche observed [158] that no component of $\mathcal{D}$ can be contained in a proper wedge (angle less than $\pi$ ). Collin 38 proved that at most one component of $\mathcal{D}$ can lie in any given half-plane. Spruck 193] demonstrated that under the assumption of sublinear growth in a suitably strong sense, $\mathcal{D}$ has at most two components. Without any assumption of the growth of the minimal graph, Li and Wang [101] proved that the number of disjointly supported minimal graphs with zero boundary values over an open subset of $\mathbb{R}^{n}$ is at most $(n+1) 2^{n+1}$. Later, Tkachev [195] improved this exponential bound by a polynomial one, and in the case $n=3$, he obtained that the number 
of disjointly supported minimal graphs is at most three. Also, Weitsman 207] has some related results that suggest that if $\mathcal{D}$ has finitely generated first homology group and $u$ has sublinear growth, then the number of components of $\mathcal{D}$ should be at most one. We refer the reader to the end of his paper [206], where he discusses several interesting unsolved problems concerning the growth of $u$ defined on a proper domain contained in a half-plane.

In the discussion of the conjectures that follow, it is helpful to fix some notation for certain classes of complete embedded minimal surfaces in $\mathbb{R}^{3}$.

- Let $\mathcal{C}$ be the space of connected, $\mathcal{C}$ omplete, embedded minimal surfaces.

- Let $\mathcal{P} \subset \mathcal{C}$ be the subspace of $\mathcal{P}$ roperly embedded surfaces.

- Let $\mathcal{M} \subset \mathcal{P}$ be the subspace of surfaces with $\mathcal{M}$ ore than one end.

Conjecture 11.5 (Finite Topology Conjecture I, Hoffman, Meeks). An orientable surface $M$ of finite topology with genus $g$ and $r$ ends, $r \neq 0,2$, occurs as a topological type of a surface in $\mathcal{C}$ if and only if $r \leq g+2$.

See [74, 79, 197, 205] and the discussion in Section 3.2 (the method of Weber and Wolf) for partial existence results which seem to indicate that the existence implication in the Finite Topology Conjecture holds when $r>2$. Recall that Theorem 1.6] insures that for each positive genus $g$, there exists an upper bound $e(g)$ on the number of ends of an $M \in \mathcal{M}$ with finite topology and genus $g$. Hence, the non-existence implication in Conjecture 11.5 will be proved if one can show that $e(g)$ can be taken as $g+2$. Concerning the case $r=2$, Theorems 1.3 and 3.1 imply that the only examples in $\mathcal{M}$ with finite topology and two ends are catenoids. Also, by Theorems 1.3 and 3.2 if $M$ has finite topology, genus zero and at least two ends, then $M$ is a catenoid.

On the other hand, one of the central results in this article (Theorem 1.1) characterizes the helicoid among complete, embedded, non-flat minimal surfaces in $\mathbb{R}^{3}$ with genus zero and one end. Concerning one-ended surfaces in $\mathcal{C}$ with finite positive genus, first note that all these surfaces are proper by Theorem 1.5. Furthermore, every example $M \in \mathcal{P}$ of finite positive genus and one end has a special analytic representation on a once-punctured compact Riemann surface, as follows from the works of Bernstein and Breiner [4] and Meeks and Pérez [115; see Theorem 1.4. In fact, these authors showed that any such minimal surface has finite typ 47 and is asymptotic to a helicoid. This finite type condition could be used to search computationally for possible examples of genus- $g$ helicoids, $g \in \mathbb{N}$. Along these lines, we have already mentioned the rigorous proofs by Hoffman, Weber and Wolf [83] and by Hoffman and White [85] of existence of a genus-one helicoid, a mathematical fact that was earlier computationally indicated by Hoffman, Karcher and Wei [76]. For genera $g=2,3,4,5,6$, there are numerical existence results [7, 8, 186, 196] as mentioned in Section 2.5 see also the last paragraph in Section 8 , All these facts motivate the next conjecture, which appeared in print for the first time in the paper [136] by Meeks and Rosenberg, although several versions of it as questions were around a long time before appearing in [136.

\footnotetext{
${ }^{47}$ A complete minimal surface $M \subset \mathbb{R}^{3}$ with Weierstrass data $(g, d h)$ is said to be of finite type if $M$ is conformally diffeomorphic to a finitely punctured, compact Riemann surface $\bar{M}$ and after a possible rotation, both $d g / g, d h$ extend meromorphically to $\bar{M}$; see Rosenberg [181] and Hauswirth, Pérez and Romon 69.
} 
Conjecture 11.6 (Finite Topology Conjecture II, Meeks, Rosenberg). For every non-negative integer $g$, there exists a unique non-planar $M \in \mathcal{C}$ with genus $g$ and one end.

The Finite Topology Conjectures I and II together propose the precise topological conditions under which a non-compact orientable surface of finite topology can be properly minimally embedded in $\mathbb{R}^{3}$. What about the case where the non-compact orientable surface $M$ has infinite topology? In this case, either $M$ has infinite genus or $M$ has an infinite number of ends. By Theorem 7.9, such an $M$ must have at most two limit ends. Theorem 10.3 states that such an $M$ cannot have one limit end and finite genus. We claim that these restrictions are the only ones.

Conjecture 11.7 (Infinite Topology Conjecture, Meeks). A non-compact, orientable surface of infinite topology occurs as a topological type of a surface in $\mathcal{P}$ if and only if it has at most one or two limit ends, and when it has one limit end, then its limit end has infinite genus.

We now discuss two conjectures related to the underlying conformal structure of a minimal surface.

Conjecture 11.8 (Liouville Conjecture, Meeks). If $M \in \mathcal{P}$ and $h: M \rightarrow \mathbb{R}$ is a positive harmonic function, then $h$ is constant.

The above conjecture is closely related to work in [39, 128, 138. For example, from the discussions in Sections 7 and 10, we know that if $M \in \mathcal{P}$ has finite genus or two limit ends, then $M$ is recurrent, which implies that $M$ satisfies the Liouville Conjecture. From results in [128, we know that the conjecture holds for all of the classical examples listed in Section 2.5. We also remark that Neel 155. proved that if a surface $M \in \mathcal{P}$ has bounded Gaussian curvature, then $M$ does not admit non-constant bounded harmonic functions. A related conjecture is the following one:

Conjecture 11.9 (Multiple-End Recurrency Conjecture, Meeks). If $M \in \mathcal{M}$, then $M$ is recurrent.

Assuming that one can prove the last conjecture, the proof of the Liouville Conjecture would reduce to the case where $M \in \mathcal{P}$ has infinite genus and one end. Note that in this setting, a surface could satisfy Conjecture 11.8 while at the same time being transient. For example, every doubly or triply periodic minimal surface with finite topology quotient satisfies the Liouville Conjecture, and these minimal surfaces are never recurrent (both properties follow from [128). On the other hand, every doubly or triply periodic minimal surface has exactly one end (Callahan, Hoffman and Meeks [16]), which implies that the assumption in Conjecture 11.9 that $M \in \mathcal{M}$, not merely $M \in \mathcal{P}$, is a necessary one. It should also be noted that the previous two conjectures need the hypothesis of global embeddedness, since there exist properly immersed minimal surfaces with two embedded ends and which admit bounded non-constant harmonic functions [39].

Conjecture 11.10 (Isometry Conjecture, Choi, Meeks, White). If $M \in \mathcal{C}$, then every intrinsic isometry of $M$ extends to an ambient isometry of $\mathbb{R}^{3}$. More generally, if $M$ is not a helicoid, then it is minimally rigid, in the sense that any isometric minimal immersion of $M$ into $\mathbb{R}^{3}$ is congruent to $M$. 
The Isometry Conjecture is known to hold if $M \in \mathcal{P}$ and either $M \in \mathcal{M}$ (Choi, Meeks and White [22]), $M$ is doubly periodic (Meeks and Rosenberg [131]), $M$ is periodic with finite topology quotient (Meeks [108] and Pérez [165]) or $M$ has finite genus (this follows from Theorem 1.4).

It can be shown that one can reduce the validity of the Isometry Conjecture to checking that whenever $M \in \mathcal{P}$ has one end and infinite genus, then there exists a plane in $\mathbb{R}^{3}$ that intersects $M$ in a set that contains a simple closed curve. If $M \in \mathcal{P}$ and there exists such a simple closed intersecting curve $\gamma$ of $M$ with a plane, then the flux of $M$ along $\gamma$ is not zero, and hence, none of the associate surfaces to $M$ are well defined (see Footnote 19 for the definition of associate surface). But Calabi 14 proved that the associate surfaces are the only isometric minimal immersions from $M$ into $\mathbb{R}^{3}$, up to congruence.

Since non-zero flux $(\mathcal{F} \neq\{0\}$ with the notation of the next conjecture) implies uniqueness of an isometric minimal immersion, the One-Flux Conjecture below implies the Isometry Conjecture.

Conjecture 11.11 (One-Flux Conjecture, Meeks, Pérez, Ros). Let $M \in \mathcal{C}$ and let $\mathcal{F}=\left\{F(\gamma)=\int_{\gamma} \operatorname{Rot}_{90^{\circ}}\left(\gamma^{\prime}\right) \mid \gamma \in H_{1}(M, \mathbb{Z})\right\}$ be the abelian group of flux vectors of $M$. If $\mathcal{F}$ has rank at most 1 , then $M$ is a plane, a helicoid, a catenoid, a Riemann minimal example or a doubly periodic Scherk minimal surface.

Conjecture 11.12 (Scherk Uniqueness Conjecture, Meeks, Wolf). If $M$ is a connected, properly immersed minimal surface in $\mathbb{R}^{3}$ and Area $(M \cap \mathbb{B}(R)) \leq 2 \pi R^{2}$ holds in balls $\mathbb{B}(R)$ of radius $R$, then $M$ is a plane, a catenoid or one of the singly periodic Scherk minimal surfaces.

By the Monotonicity Formula, any connected, properly immersed minimal surface in $\mathbb{R}^{3}$ with

$$
\lim _{R \rightarrow \infty} R^{-2} \operatorname{Area}(M \cap \mathbb{B}(R)) \leq 2 \pi
$$

is actually embedded. A related conjecture on the uniqueness of the doubly periodic Scherk minimal surfaces was solved by Lazard-Holly and Meeks [100; they proved that if $M \in \mathcal{P}$ is doubly periodic and its quotient surface has genus zero, then $M$ is one of the doubly periodic Scherk minimal surfaces. The basic approach used in [100] was adapted later on by Meeks and Wolf [143] to prove that Conjecture 11.12 holds under the assumption that the surface is singly periodic.

Conjecture 11.13 (Unique Limit Tangent Cone at Infinity Conjecture, Meeks). If $M \in \mathcal{P}$ is not a plane and has quadratic area growth, then $\lim _{t \rightarrow \infty} \frac{1}{t} M$ exists and is a minimal, possibly non-smooth cone over a finite balanced configuration of geodesic arcs in the unit sphere, with common end points and integer multiplicities. Furthermore, if $M$ has area not greater than $2 \pi R^{2}$ in balls of radius $R$, then the limit tangent cone of $M$ is either the union of two planes or consists of a single plane with multiplicity two passing through the origin.

By unpublished work of Meeks and Wolf, the above conjecture is closely tied to the validity of the next classical one.

Conjecture 11.14. Let $f: M \rightarrow \mathbb{B}-\{\overrightarrow{0}\}$ be a proper immersion of a surface with compact boundary in the punctured unit ball, such that $f(\partial M) \subset \partial \mathbb{B}$ and whose mean curvature function is bounded. Then $f(M)$ has a unique limit tangent cone at the origin under homothetic expansions. 
If $M \in \mathcal{C}$ has finite topology, then $M$ has finite total curvature or is asymptotic to a helicoid by Theorems 1.3, 1.4 and 1.5. It follows that for any such surface $M$, there exists a constant $C_{M}>0$ such that the injectivity radius function $I_{M}: M \rightarrow(0, \infty]$ satisfies

$$
I_{M}(p) \geq C_{M}\|p\|, \quad p \in M .
$$

Work of Meeks, Pérez and Ros in [121, 122 indicates that this linear growth property of the injectivity radius function should characterize the examples in $\mathcal{C}$ with finite topology.

Conjecture 11.15 (Injectivity Radius Growth Conjecture, Meeks, Pérez, Ros). A surface $M \in \mathcal{C}$ has finite topology if and only if its injectivity radius function grows at least linearly with respect to the extrinsic distance from the origin.

The results in [121, 122] and the earlier described Theorems 4.2 and 4.7 also motivated several conjectures concerning the limits of locally simply connected sequences of minimal surfaces in $\mathbb{R}^{3}$, such as the following one. See the monograph [116 for partial results on this conjecture.

Conjecture 11.16 (Parking Garage Structure Conjecture, Meeks, Pérez, Ros). Suppose $M_{n} \subset \mathbb{B}\left(R_{n}\right)$ is a locally simply connected sequence of embedded minimal surfaces with $\partial M_{n} \subset \partial \mathbb{B}\left(R_{n}\right)$ and $R_{n} \rightarrow \infty$ as $n \rightarrow \infty$. Assume also that the sequence $M_{n}$ does not have uniformly bounded curvature in $\mathbb{B}(1)$. Then:

1. After a rotation and choosing a subsequence, the $M_{n}$ converge to a minimal parking garage structure on $\mathbb{R}^{3}$ consisting of the foliation $\mathcal{L}$ of $\mathbb{R}^{3}$ by horizontal planes, with singular set of convergence being a locally finite collection $\mathcal{S}(\mathcal{L})$ of vertical lines which are the columns of the parking garage structure.

2. For any two points $p, q \in \mathbb{R}^{3}-\mathcal{S}(\mathcal{L})$, the ratio of the vertical spacing between consecutive sheets of the double multigraphs defined by $M_{n}$ near $p$ and $q$ converges to one as $n \rightarrow \infty$. Equivalently, the Gaussian curvature of the sequence $M_{n}$ blows up at the same rate along all the columns as $n \rightarrow \infty$.

We next deal with the question of when a surface $M \in \mathcal{C}$ has strictly negative Gaussian curvature. Suppose again that a surface $M \in \mathcal{C}$ has finite topology, and so, $M$ either has finite total curvature or is a helicoid with handles. It is straightforward to check that such a surface has negative curvature if and only if it is a catenoid or a helicoid (note that if $g: M \rightarrow \mathbb{C} \cup\{\infty\}$ is the stereographically projected Gauss map of $M$, then after a suitable rotation of $M$ in $\mathbb{R}^{3}$, the meromorphic differential $\frac{d g}{g}$ vanishes exactly at the zeros of the Gaussian curvature of $M$; from here one deduces easily that if $M$ has finite topology and strictly negative Gaussian curvature, then the genus of $M$ is zero). More generally, if we allow a surface $M \in \mathcal{C}$ to be invariant under a proper discontinuous group $G$ of isometries of $\mathbb{R}^{3}$, with $M / G$ having finite topology, then $M / G$ is properly embedded in $\mathbb{R}^{3} / G$ by an elementary application of the Minimal Lamination Closure Theorem (see Proposition 1.3 in [172]). Hence, in this case, $M / G$ has finite total curvature by a result of Meeks and Rosenberg [131, 134. Suppose additionally that $M / G$ has negative curvature, and we will discuss which surfaces are possible. If the ends of $M / G$ are helicoidal or planar, then a similar argument using $\frac{d g}{g}$ gives that $M$ has genus zero, and so, it is a helicoid. If $M / G$ is doubly periodic, then $M$ is a Scherk minimal surface; see 123 . In the case that $M / G$ is singly periodic, then 
$M$ must have Scherk-type ends, but we still do not know if the surface must be a Scherk singly periodic minimal surface. These considerations motivate the following conjecture.

Conjecture 11.17 (Negative Curvature Conjecture, Meeks, Pérez, Ros). If $M \in \mathcal{C}$ has negative curvature, then $M$ is a catenoid, a helicoid or one of the singly or doubly periodic Scherk minimal surfaces.

Passing to a different question, some of the techniques developed by Meeks, Pérez and Ros in 122 provide a beginning theory for analyzing and possibly characterizing examples in $\mathcal{C}$ whose Gauss maps exclude two or more points on $\mathbb{S}^{2}$. A classical result of Fujimoto 63. establishes that the Gauss map of any orientable, complete, non-flat, minimally immersed surface in $\mathbb{R}^{3}$ cannot exclude more than 4 points, which improved the earlier result of Xavier 208 that the Gauss map of such a surface cannot miss more than 6 points. If one assumes that a surface $M \in \mathcal{C}$ is periodic with finite topology quotient, then Meeks, Pérez and Ros solved the next conjecture [123. Also see Kawakami, Kobayashi and Miyaoka 95] for related results on this problem, including some partial results on the conjecture of Osserman that states that the Gauss map of an orientable, complete, non-flat, immersed minimal surface with finite total curvature in $\mathbb{R}^{3}$ cannot miss 3 points of $\mathbb{S}^{2}$.

Conjecture 11.18 (Four Point Conjecture, Meeks, Pérez, Ros). If $M \in \mathcal{C}$ and the Gauss map of $M$ omits four points on $\mathbb{S}^{2}$, then $M$ is a singly or doubly periodic Scherk minimal surface.

The following three conjectures are related to the embedded Calabi-Yau problem.

Conjecture 11.19 (Finite Genus Properness Conjecture, Meeks, Pérez, Ros). If $M \in \mathcal{C}$ and $M$ has finite genus, then $M \in \mathcal{P}$.

In [120], Meeks, Pérez and Ros proved Conjecture 11.19 under the additional hypothesis that $M$ has a countable number of ends (recall that this assumption is necessary for $M$ to be proper in $\mathbb{R}^{3}$ by Theorem 7.9 . A stronger conjecture by Meeks, Pérez and Ros [126] states that if $M \in \mathcal{C}$ has finite genus, then $M$ has bounded Gaussian curvature; note that Theorem 5.8 implies that if $M \in \mathcal{C}$ has locally bounded Gaussian curvature in $\mathbb{R}^{3}$ and finite genus, then $M$ is properly embedded.

Conjecture 11.20 (Embedded Calabi-Yau Conjectures, Martín, Meeks, Nadirashvili, Pérez, Ros).

1. There exists an $M \in \mathcal{C}$ whose closure $\bar{M}$ has the structure of a minimal lamination of a slab, with $M$ as a leaf and with two planes as limit leaves. In particular, $\mathcal{P} \neq \mathcal{C}$.

2. A necessary and sufficient condition for a connected, open topological surface $M$ to admit a complete bounded minimal embedding in $\mathbb{R}^{3}$ is that every end of $M$ has infinite genus.

3. A necessary and sufficient condition for a connected, open topological surface $M$ to admit a proper minimal embedding in every smooth bounded domain $\mathcal{D} \subset \mathbb{R}^{3}$ as a complete surface is that $M$ is orientable and every end of $M$ has infinite genus.

4. A necessary and sufficient condition for a connected, non-orientable open topological surface $M$ to admit a proper minimal embedding in some bounded 
domain $\mathcal{D} \subset \mathbb{R}^{3}$ as a complete surface is that every end of $M$ has infinite genus.

The next conjecture deals with the embedded Calabi-Yau problem in a Riemannian three-manifold $N$. We remark that Conjecture 11.19 can be shown to follow from the next conjecture.

Conjecture 11.21 (Finite Genus Conjecture in three-manifolds, Meeks, Pérez, Ros). Suppose $M$ is a connected, complete, embedded minimal surface with empty boundary and finite genus in a Riemannian three-manifold $N$. Let $\bar{M}=M \cup L(M)$, where $L(M)$ is the set of limit point 48 of $M$. Then, one of the following possibilities holds.

1. $\bar{M}$ has the structure of a minimal lamination of $N$.

2. $\bar{M}$ fails to have a minimal lamination structure, $L(M)$ is a non-empty minimal lamination of $N$ consisting of stable leaves and $M$ is properly embedded in $N-L(M)$.

Using arguments contained in the proofs of Theorems 1.5 and 9.2 , we believe that Conjecture 11.21 can be reduced to be a consequence of the next conjecture, which is motivated by partial results in [37, 120. Recall that given $\delta>0$, we let $\mathbb{B}(\delta)=\left\{x \in \mathbb{R}^{3}|| x \mid<\delta\right\}$.

Conjecture 11.22 (Chord-Arc Conjecture, Meeks, Pérez, Ros). For any $k \geq 0$, there exists a positive number $\delta(k)<\frac{1}{2}$ such that if $\Sigma$ is a compact, embedded minimal surface with genus $k$ in the closed unit ball $\overline{\mathbb{B}} \subset \mathbb{R}^{3}$ with $\partial \Sigma \subset \partial \mathbb{B}$ and $\overrightarrow{0} \in \Sigma$, then the component $\Sigma(\overrightarrow{0}, \delta(k))$ of $\Sigma \cap \mathbb{B}(\delta(k))$ containing $\overrightarrow{0}$ satisfies

$$
\Sigma(\overrightarrow{0}, \delta(k)) \subset B_{\Sigma}\left(\overrightarrow{0}, \frac{1}{2}\right),
$$

where $B_{\Sigma}\left(\overrightarrow{0}, \frac{1}{2}\right)$ is the intrinsic ball in $\Sigma$ of radius $\frac{1}{2}$ centered at the point $\overrightarrow{0} \in \Sigma$.

Our next problem is related to integral curves of harmonic functions. Theorem 7.7 implies that for any properly immersed minimal surface $M$ in $\mathbb{R}^{3}$ and for any $t \in \mathbb{R}$, the surface $M(t)=M \cap\left\{x_{3} \leq t\right\}$ is parabolic. In [113, Meeks used the parabolicity of $M(t)$ to show that the scalar flux of $\nabla x_{3}$ across $\partial M(t)$ does not depend on $t$ (this result is called the Algebraic Flux Lemma). If $M$ were recurrent, then it is known (Tsuji 202]) that the following stronger property holds: almost all integral curves of $\nabla x_{3}$ begin at $x_{3}=-\infty$ and end at $x_{3}=\infty$. The next conjecture is a strengthening of this geometric flux property to arbitrary properly embedded minimal surfaces in $\mathbb{R}^{3}$.

Conjecture 11.23 (Geometric Flux Conjecture, Meeks, Rosenberg). Let $M \in \mathcal{P}$ and $h: M \rightarrow \mathbb{R}$ be a non-constant coordinate function on $M$. Consider the set $I$ of integral curves of $\nabla h$. Then there exists a countable set $C \subset I$ such that for any integral curve $\alpha \in I-C$, the composition $h \circ \alpha: \mathbb{R} \rightarrow \mathbb{R}$ is a diffeomorphism 49

We have seen examples of how one can produce stable minimal surfaces by using barrier constructions (Section 2.9), and how these stable minimal surfaces act as guide posts which are useful for deciphering the structure of complete, embedded minimal surfaces (e.g., in the proofs of Theorems 2.31 and 6.2). Below, we have

\footnotetext{
${ }^{48}$ See the paragraph just before Theorem 9.2 for the definition of $L(M)$.

${ }^{49}$ After a choice of $p \in \alpha$, we are identifying $\alpha$ with the parameterized curve $\alpha: \mathbb{R} \rightarrow M$ such that $\alpha(0)=p$ and $\alpha^{\prime}(t)=\nabla h(\alpha(t)), t \in \mathbb{R}$.
} 
collected some outstanding problems that concern stable minimal surfaces. Regarding item 1 in the next conjecture, Ros (unpublished) proved that a complete, non-orientable minimal surface without boundary which is stable outside a compact set50 must have finite total curvature. The validity of item 2 implies that the sublamination of limit leaves of the lamination $\mathcal{L}$ in Conjecture 11.3 extends to a lamination of $\mathbb{R}^{3}$ by planes. In reference to item 3 , we remark that complete, stable minimal surfaces with boundary are not in general parabolic (see pages 22 and 23 in our survey [117). Concerning item 4, Pérez [168 proved this conjecture under the additional assumptions that the surface is proper and has quadratic area growth.

Conjecture 11.24 (Stable Minimal Surface Conjectures). 1. A complete, non-orientable, stable minimal surface in $\mathbb{R}^{3}$ with compact boundary has finite total curvature (Ros).

2. If $A \subset \mathbb{R}^{3}$ is a closed set with zero 1-dimensional Hausdorff measure and $M \subset \mathbb{R}^{3}-A$ is a connected, stable, minimally immersed surface which is complete outside of $A$, then the closure of $M$ is a plane (Meeks).

3. If $M \subset \mathbb{R}^{3}$ is a complete, stable minimal surface with boundary, then $M$ is $\delta$-parabolic, i.e., given $\delta>0$, the set $M(\delta)=\left\{p \in M \mid \operatorname{dist}_{M}(p, \partial M) \geq \delta\right\}$ is parabolic (Meeks, Rosenberg).

4. A complete, embedded, stable minimal surface in $\mathbb{R}^{3}$ with boundary a straight line is a half-plane, a half of the Enneper minimal surface or a half of the helicoid (Pérez, Ros, White).

Any end of a surface $M \in \mathcal{C}$ with finite total curvature is $C^{2}$-asymptotic to the end of a plane or catenoid (equation (10)). Our last conjecture can be viewed as a potential generalization of this result.

Conjecture 11.25 (Standard Middle End Conjecture, Meeks). If $M \in \mathcal{M}$ and $E \subset M$ is a one-ended representative for a middle end of $M$, then $E$ is $C^{0}$ asymptotic to the end of a plane or catenoid. In particular, if $M$ has two limit ends, then each middle end is $C^{0}$-asymptotic to a plane.

For a non-annular, one-ended middle end representative $E$ (i.e., $E$ has infinite genus) in the above conjecture, $\lim _{t \rightarrow \infty} \frac{1}{t} E$ is a plane $P$ passing through the origin with positive integer multiplicity at least two by Theorem 7.9. Also, if $M$ has two limit ends and horizontal limit tangent plane at infinity, then for such a middle end representative $E$, every divergent sequence of horizontal translates of $E$ has a subsequence which converges to a finite collection of horizontal planes. This limit collection might depend on the sequence; in this case, it remains to prove there is only one plane in limit collections of this type.

\section{ABOUT THE AUTHORS}

William H. Meeks III is the George David Birkhoff Professor of Mathematics at the University of Massachusetts at Amherst, and Joaquín Pérez is a professor of the Department of Geometry and Topology at the University of Granada (Spain). They have collaborated since 1998 with common research interests in minimal and constant mean curvature surface theory in three-manifolds.

\footnotetext{
${ }^{50}$ See Footnote 21 for the definition of stability in the non-orientable case.
} 


\section{ACKNOWLEDGMENTS}

The authors would like to thank Markus Schmies, Martin Traizet, Matthias Weber, the Scientific Graphics Project and the 3D-XplorMath Surface Gallery for contributing the beautiful computer graphics images to Section 2.5 of examples of classical minimal surfaces. We would like to thank Tobias Colding, David Hoffman, Nicos Kapouleas, Hermann Karcher, Rob Kusner, Francisco Martín, Bill Minicozzi, Nikolai Nadirashvili, Bob Osserman, Antonio Ros, Martin Traizet, Matthias Weber, Allen Weitsman and Mike Wolf for detailed suggestions on improving this article. Finally, we wish to thank the referee for comments and criticisms that have contributed to the clarity of the exposition.

\section{REFERENCES}

1. A. Alarcón, L. Ferrer, and F. Martín, Density theorems for complete minimal surfaces in $\mathbb{R}^{3}$, Geom. Funct. Anal. 18 (2008), no. 1, 1-49. MR2399094

2. A. D. Alexandrov, Uniqueness theorems for surfaces in the large I, Vestnik Leningrad Univ. Math. 11 (1956), no. 19, 5-17. MR0150706

3. D. Anderson, C. Henke, D. Hoffman, and E. L. Thomas, Periodic area-minimizing surfaces in block copolymers, Nature 334 (1988), no. 6184, 598-601, August 18 issue.

4. J. Bernstein and C. Breiner, Conformal structure of minimal surfaces with finite topology, Preprint available at http://arxiv.org/abs/0810.4478v1.

5. _ Helicoid-like minimal disks and uniqueness, Preprint available at http://arxiv.org/ abs/0802.1497.

6. S. Bernstein, Uber ein geometrisches Theorem und seine Anwendung auf die partiellen Differentialgleichungen vom elliptischen Typus, Math. Z. 26 (1927). 551-558. MR.1544873

7. A. I. Bobenko, Helicoids with handles and Baker-Akhiezer spinors, Math. Z. 229 (1998), no. 1, 9-29. MR1649381

8. A. I. Bobenko and M. Schmies, Computer graphics experiments for helicoids with handles, Personal communication.

9. F. Bonahon, Geometric structures on 3-manifolds, Handbook of geometric topology, NorthHolland, Amsterdam, 2002, pp. 93-164. MR.1886669 (2003b:57021)

10. O. Bonnet, Mémoire sur l'emploi d'un nouveau système de variables dans l'étude des surfaces courbes, J. Mathemém. p. appl. 2 (1860), 153-266.

11. J. C. Borda, Eclaircissement sur les méthodes de trouver les courbes qui jouissent de quelque propiété du maximum ou du minimum, Mém. Acad. Roy. Sci. Paris (1770), 551-565, Presented in 1767.

12. C. V. Boys, Soap bubbles: Their colours and the forces which mold them, Dover Publications, New York, 1959.

13. E. Calabi, Problems in differential geometry, Proceedings of the United States-Japan Seminar in Differential Geometry, Kyoto, Japan 1965, Nippon Hyoronsha Co. Ltd., Tokyo, 1966, p. 170. MR0216513(35:7346)

14. _ Quelques applications de l'analyse complexe aux surfaces d'aire minima, Topics in Complex Manifolds, Les Presses de l'Université de Montréal, 1967, H. Rossi, editor, pp. 5981.

15. M. Callahan, D. Hoffman, and W. H. Meeks III, Embedded minimal surfaces with an infinite number of ends, Invent. Math. 96 (1989), 459-505. MR0996552

16. _ The structure of singly-periodic minimal surfaces, Invent. Math. 99 (1990), 455-481. MR.1032877

17. E. Catalan, Sur les surfaces réglées dont l'aire est un minimum, J. Mathem. p. appl. 7 (1842), 203-211.

18. I. Chavel, Riemannian geometry: a modern introduction, Cambridge University Press, 1993. MR.1271141

19. C. C. Chen and F. Gackstatter, Elliptic and hyperelliptic functions and complete minimal surfaces with handles, Instituto de Matemática e Estatistica-Universidade de São Paulo 27 (1981). 
20. __ Elliptische und Hyperelliptische Functionen und vollständige Minimalflächen von Enneperschen Typ, Math. Ann. 259 (1982), 359-369. MR0661204

21. S. S. Chern, The geometry of G-structures, Bull. Amer. Math. Soc. 72 (1966), 167-219. MR 0192436

22. T. Choi, W. H. Meeks III, and B. White, A rigidity theorem for properly embedded minimal surfaces in $\mathbb{R}^{3}$, J. Differential Geom. 32 (1990), 65-76. MR1064865

23. T. H. Colding, C. de Lellis, and W. P. Minicozzi II, Three circles theorems for Schrödinger operators on cylindrical ends and geometric applications, Comm. Pure Appl. Math. 61 (2008), no. 11, 1540-1602. MR2444375

24. T. H. Colding and W. P. Minicozzi II, The space of embedded minimal surfaces of fixed genus in a 3-manifold V; Fixed genus, Preprint math.DG/0509647 (2005).

25. _ Minimal surfaces, Courant Lecture Notes in Mathematics, vol. 4, New York University Courant Institute of Mathematical Sciences, New York, 1999. MR1683966

26. - Complete properly embedded minimal surfaces in $\mathbb{R}^{3}$, Duke Math. J. 107 (2001), 421-426. MR:1823052

27. _ Multivalued minimal graphs and properness of disks, International Mathematical Research Notices 21 (2002), 1111-1127. MR.1904463

28. Disks that are double spiral staircases, Notices of the AMS 50 (2003), no. 3, 327-339. MR.1954009

29. _ـ Embedded minimal disks: proper versus nonproper - global versus local, Transactions of the AMS 356 (2003), no. 1, 283-289. MR2020033

30. , An excursion into geometric analysis, Surveys of Differential Geometry IX. Eigenvalues of Laplacian and other geometric operators, International Press, edited by Alexander Grigor'yan and Shing Tung Yau, 2004, pp. 83-146. MR2195407

31. _ The space of embedded minimal surfaces of fixed genus in a 3-manifold I; Estimates off the axis for disks, Ann. of Math. (2) 160 (2004), 27-68. MR2119717

32. _ - The space of embedded minimal surfaces of fixed genus in a 3-manifold II; Multivalued graphs in disks, Ann. of Math. (2) 160 (2004), 69-92. MR2119718

33. domains, Ann. of Math. (2) 160 (2004), 523-572. MR2123932

34. - The space of embedded minimal surfaces of fixed genus in a 3-manifold IV; Locally simply-connected, Ann. of Math. (2) 160 (2004), 573-615. MR2123933

35. _ Embedded minimal disks, Global theory of minimal surfaces, American Mathematical Society, Providence, RI, for the Clay Mathematics Institute, Cambridge, MA, edited by D. Hoffman, 2005, pp. 405-438. MR2167253

36. _ Shapes of embedded minimal surfaces, Proc. National Academy of Sciences 103 (2006), 11106-11111. MR2242650

37. The Calabi-Yau conjectures for embedded surfaces, Ann. of Math. (2) 167 (2008), 211-243. MR 2373154

38. P. Collin, Topologie et courbure des surfaces minimales de $\mathbb{R}^{3}$, Ann. of Math. (2) 145-1 (1997), 1-31. MR1432035

39. P. Collin, R. Kusner, W. H. Meeks III, and H. Rosenberg, The geometry, conformal structure and topology of minimal surfaces with infinite topology, J. Differential Geom. 67 (2004), 377393. MR 2153082

40. B. Coskunuzer, Non-properly embedded minimal planes in hyperbolic 3-space, Preprint, 2011.

41. C. Costa, Imersöes minimas en $\mathbb{R}^{3}$ de gênero un e curvatura total finita, Ph.D. thesis, IMPA, Rio de Janeiro, Brasil, 1982

42. __ Example of a complete minimal immersion in $\mathbb{R}^{3}$ of genus one and three embedded ends, Bull. Soc. Bras. Mat. 15 (1984), 47-54. MR0794728

43. _ Uniqueness of minimal surfaces embedded in $\mathbb{R}^{3}$ with total curvature $12 \pi$, J. Differential Geom. 30 (1989), no. 3, 597-618. MR1021368

44. Classification of complete minimal surfaces in $\mathbb{R}^{3}$ with total curvature $12 \pi$, Invent. Math. 105 (1991), no. 2, 273-303. MR1115544

45. R. Courant, Soap film experiments with minimal surfaces, Amer. Math. Monthly 47 (1940), 167-174. MR 0001622

46. Dr. Crypton, Shapes that eluded discovery, Science Digest (1986), 50-55, 78.

47. U. Dierkes, S. Hildebrandt, A. Küster, and O. Wohlrab, Minimal surfaces I, Grundlehren der mathematischen Wissenschaften 295, Springer-Verlag, 1992. MR1215267 
48. _ Minimal surfaces II, Grundlehren der mathematischen Wissenschaften 296, Springer-Verlag, 1992. MR1215268

49. M. do Carmo and C. K. Peng, Stable complete minimal surfaces in $\mathbb{R}^{3}$ are planes, Bulletin of the AMS 1 (1979), 903-906. MR0546314

50. G. Donnay and D. L. Pawson, X-ray diffraction studies of echinoderm plates, Science 166 (1969), 1147-1150.

51. T. Ekholm, B. White, and D. Wienholtz, Embeddedness of minimal surfaces with total

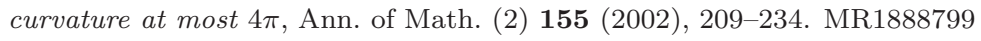

52. A. Enneper, Analytisch-geometrische Untersuchungen, Z. Math. und Phys. 9 (1864), 96-125.

53. L. Euler, Methodus inveniendi lineas curvas maximi minimive propietate gaudeates sive solutio problematis isoperimetrici latissimo sensu accepti, Harvard Univ. Press, Cambridge, MA, 1969, Opera omnia(1), 24, Fussli, Turici, 1952. A source book in mathematics, partially translated by D. J. Struik, see pages 399-406.

54. Y. Fang, On minimal annuli in a slab, Comment. Math. Helv. 69 (1994), no. 3, 417-430. MR 1289335

55. - Minimal annuli in $\mathbb{R}^{3}$ bounded by non-compact complete convex curves in parallel planes, J. Austral. Math. Soc. Ser. A 60 (1996), no. 3, 369-388. MR1385149

56. H. Federer, Geometric measure theory, Springer-Verlag, Berlin-Heidelberg, New York, 1969. MR 0257325

57. I. Fernández and P. Mira, Holomorphic quadratic differentials and the Bernstein problem in Heisenberg space, Trans. Amer. Math. Soc. 361 (2009), 5737-5752. MR2529912

58. L. Ferrer, F. Martín, and W. H. Meeks III, The existence of proper minimal surfaces of arbitrary topological type, Preprint available at arXiv.org/abs/0903.4194.

59. D. Fischer-Colbrie, On complete minimal surfaces with finite Morse index in 3-manifolds, Invent. Math. 82 (1985), 121-132. MR0808112

60. D. Fischer-Colbrie and R. Schoen, The structure of complete stable minimal surfaces in 3-manifolds of nonnegative scalar curvature, Comm. on Pure and Appl. Math. 33 (1980), 199-211. MR 0562550

61. C. Frohman and W. H. Meeks III, The ordering theorem for the ends of properly embedded minimal surfaces, Topology 36 (1997), no. 3, 605-617. MR1422427

62. C. Frohman and W. H. Meeks III, The topological classification of minimal surfaces in $\mathbb{R}^{3}$, Ann. of Math. (2) 167 (2008), no. 3, 681-700. MR2415385

63. H. Fujimoto, On the number of exceptional values of the Gauss maps of minimal surfaces, J. of the Math. Society of Japan 40 (1988), no. 2, 235-247. MR0930599

64. F. Gackstatter, Über die Dimension einer Minimalfläche und zur Ungleichung von St. CohnVossen, Arch. Rational Mech. Anal. 61 (1976), no. 2, 141-152. MR0420447

65. F. Gesztesy and R. Weikard, Elliptic algebro-geometric solutions of the KdV and AKNS hierarchies - an analytic approach, Bull. Amer. Math. Soc. (N.S.) 35 (1998), no. 4, 271-317. MR 1638298 (99i:58075)

66. A. Grigor'yan, Analytic and geometric background of recurrence and non-explosion of Brownian motion on Riemannian manifolds, Bull. of A.M.S 36 (1999), no. 2, 135-249. MR 1659871

67. A. Grigor'yan, Y. Netrusov, and S. T. Yau, Eigenvalues of elliptic operators and geometric applications, Surveys of Differential Geometry IX, International Press, 2004, pp. 147-218. MR 2195408

68. R. Gulliver and H. B. Lawson, The structure of minimal hypersurfaces near a singularity, Proc. Symp. Pure Math. 44 (1986), 213-237. MR.0840275

69. L. Hauswirth, J. Pérez, and P. Romon, Embedded minimal ends of finite type, Transactions of the AMS 353 (2001), 1335-1370. MR1806738

70. S. Hildebrandt, Boundary behavior of minimal surfaces, Archive Rational Mech. Anal. 35 (1969), 47-81. MR0248650

71. The calculus of variations today, Mathematical Intelligencer 11 (1989), no. 4, 50-60. MR 1016107

72. D. Hoffman, The computer-aided discovery of new embedded minimal surfaces, Mathematical Intelligencer 9 (1987), no. 3, 8-21. MR0895770

73. __ Computing minimal surfaces, Global theory of minimal surfaces, American Mathematical Society, Providence, RI, for the Clay Mathematics Institute, Cambridge, MA, edited by D. Hoffman, 2005, pp. 259-282. MR 2167253 
74. D. Hoffman and H. Karcher, Complete embedded minimal surfaces of finite total curvature, Encyclopedia of Mathematics, Vol. 90, Geometry V (R. Osserman, ed.), Springer-Verlag, 1997, pp. 5-93. MR1490038

75. D. Hoffman, H. Karcher, and F. Wei, Adding handles to the helicoid, Bulletin of the AMS, New Series 29 (1993), no. 1, 77-84. MR.1193537.

76. _ The genus one helicoid and the minimal surfaces that led to its discovery, Global Analysis and Modern Mathematics, Publish or Perish Press, 1993, K. Uhlenbeck, editor, pp. 119-170. MR.1278754

77. _ The singly periodic genus-one helicoid, Comment. Math. Helv. 74 (1999), 248-279. MR 1691949

78. D. Hoffman and W. H. Meeks III, A complete embedded minimal surface in $\mathbb{R}^{3}$ with genus one and three ends, J. Differential Geom. 21 (1985), 109-127. MR0806705

79. _ Embedded minimal surfaces of finite topology, Ann. of Math. (2) 131 (1990), 1-34. MR 1038356

80. (1990), no. 2, 181-195. MR1057654

81. , Minimal surfaces based on the catenoid, Amer. Math. Monthly, Special Geometry Issue 97 (1990), no. 8, 702-730. MR.1072813

82. $\quad$ The strong halfspace theorem for minimal surfaces, Invent. Math. 101 (1990), 373377. MR 1062966

83. D. Hoffman, M. Weber, and M. Wolf, An embedded genus-one helicoid, Ann. of Math. (2) 169 (2009), no. 2, 347-448. MR2480608

84. D. Hoffman and F. Wei, Deforming the singly periodic genus-one helicoid, Experimental Mathematics 11 (2002), no. 2, 207-218. MR1959264

85. D. Hoffman and B. White, Genus-one helicoids from a variational point of view, Comm. Math. Helv. 83 (2008), no. 4, 767-813. MR2442963 (2010b:53013)

86. H. Hopf, Differential geometry in the large, Lecture Notes in Math., vol. 1000, SpringerVerlag, 1989. MR:1013786

87. A. Huber, On subharmonic functions and differential geometry in the large, Comment. Math. Helvetici 32 (1957), 181-206. MR0094452

88. Y. Imayoshi and M. Taniguchi (eds.), An introduction to Teichmüller spaces, Springer-Verlag, 1992. MR 1215481

89. L. Jorge and W. H. Meeks III, The topology of complete minimal surfaces of finite total Gaussian curvature, Topology 22 (1983), no. 2, 203-221. MR0683761

90. N. Kapouleas, Complete embedded minimal surfaces of finite total curvature, J. Differential Geom. 47 (1997), no. 1, 95-169. MR1601434

91. _ Constructions of minimal surfaces by gluing minimal immersions, Global theory of minimal surfaces, American Mathematical Society, Providence, RI, for the Clay Mathematics Institute, Cambridge, MA, edited by D. Hoffman, 2005, pp. 489-524. MR 2167253

92. H. Karcher, Embedded minimal surfaces derived from Scherk's examples, Manuscripta Math. 62 (1988), 83-114. MR0958255

93. Construction of minimal surfaces, Surveys in Geometry (1989), 1-96, University of Tokyo, 1989, and Lecture Notes No. 12, SFB256, Bonn, 1989.

94. The triply periodic minimal surfaces of Alan Schoen and their constant mean curvature companions, Manuscripta Math. 64 (1989), 291-357. MR 1003093

95. Y. Kawakami, R. Kobayashi, and R. Miyaoka, The Gauss map of pseudo-algebraic minimal surfaces, Mathematische Nachrichten 282 (2009), no. 2, 157-306. MR 2479289 (2009m:53020)

96. N. Korevaar, R. Kusner, and B. Solomon, The structure of complete embedded surfaces with constant mean curvature, J. Differential Geom. 30 (1989), 465-503. MR.1010168

97. J. L. Lagrange, Essai d'une nouvelle méthode pour determiner les maxima et les minima des formules integrales indefinies, Miscellanea Taurinensia 2325 (1760), no. 1, 173-199.

98. R. Langevin and H. Rosenberg, A maximum principle at infinity for minimal surfaces and applications, Duke Math. J. 57 (1988), no. 3, 819-828. MR0975123

99. H. B. Lawson, Jr., Lectures on minimal submanifolds, Publish or Perish Press, Berkeley, 1980. MR0576752 
100. H. Lazard-Holly and W. H. Meeks III, Classification des surfaces minimales de genre zéro proprement plongées dans $\mathbb{R}^{3} / \mathbb{Z}^{2}$, Comptes Rendus de l'Académie des Sciences de Paris (1997), 753-754. MR.1483712

101. P. Li and J. Wang, Finiteness of disjoint minimal graphs, Math. Research Letters 8 (2001), no. 6, 771-777. MR 1879819

102. R. B. Lockhart and R. C. McOwen, Elliptic differential operators on noncompact manifolds, Ann. Scuola Norm. Sup. Pisa 12 (1985), no. 3, 409-447. MR0837256

103. F. J. López and F. Martín, Complete minimal surfaces in $\mathbb{R}^{3}$, Publ. Mat. 43 (1999), no. 2, 341-449. MR 1744617

104. F. J. López and A. Ros, On embedded complete minimal surfaces of genus zero, J. Differential Geom. 33 (1991), no. 1, 293-300. MR1085145

105. W. H. Meeks III, Lectures on Plateau's problem, Instituto de Matematica Pura e Aplicada (IMPA), Rio de Janeiro, Brazil, 1978.

106. _ The classification of complete minimal surfaces with total curvature greater than $-8 \pi$, Duke Math. J. 48 (1981), 523-535. MR0630583

107. _ A survey of the geometric results in the classical theory of minimal surfaces, Bol. Soc. Brasil Mat. 12 (1981), 29-86. MR0671473

108. _ The theory of triply-periodic minimal surfaces, Indiana Univ. Math. J. 39 (1990), no. 3, 877-936. MR1078743

109. The geometry, topology, and existence of periodic minimal surfaces, Proceedings of Symposia in Pure Math. 54 (1993), 333-374, Part I. MR.1216594

110. , Geometric results in classical minimal surface theory, Surveys in Differential Geometry, vol. 8, International Press, edited by S.T. Yau, 2003, pp. 269-306. MR2039993

111. The regularity of the singular set in the Colding and Minicozzi lamination theorem, Duke Math. J. 123 (2004), no. 2, 329-334. MR2066941

112. _ The limit lamination metric for the Colding-Minicozzi minimal lamination, Illinois J. of Math., 49 (2005) no. 2, 645-658. MR2164355 (2006e:53021)

113. Global problems in classical minimal surface theory, Global theory of minimal surfaces, American Mathematical Society, Providence, RI, for the Clay Mathematics Institute, Cambridge, MA, edited by D. Hoffman, 2005, pp. 453-470. MR2167253

114. _ Proofs of some classical results in minimal surface theory, Indiana J. of Math. $\mathbf{5 4}$ (2005), no. 4, 1031-1045. MR2164416

115. W. H. Meeks III and J. Pérez, Embedded minimal surfaces of finite topology, Preprint available at http://www.ugr.es/local/jperez/papers/papers.htm.

116. _ A survey on classical minimal surface theory, Preprint available at http://www.ugr.es/local/jperez/papers/papers.htm.

117. _ Conformal properties in classical minimal surface theory, Surveys of Differential Geometry IX - Eigenvalues of Laplacian and other geometric operators, International Press, edited by Alexander Grigor'yan and Shing Tung Yau, 2004, pp. 275-336. MR.2195411

118. _ Properly embedded minimal planar domains with infinite topology are Riemann minimal examples, Current Developments in Mathematics 2008 Conference Proceedings, International Press, edited by David Jenson, Barry Mazur, Tornasz Mrowka, Wilfried Schmid, Richard P. Stanley, Shing-Tung Yau, 2008.

119. W. H. Meeks III, J. Pérez, and A. Ros, Bounds on the topology and index of classical minimal surfaces, Preprint available at http://www.ugr.es/local/jperez/papers/papers.htm.

120. _ The embedded Calabi-Yau conjectures for finite genus, Preprint available at http://www.ugr.es/local/jperez/papers/papers.htm.

121. _ The local picture theorem on the scale of topology, Preprint available at http://www.ugr.es/local/jperez/papers/papers.htm.

122. L Local removable singularity theorems for minimal and H-laminations, Preprint available at http://www.ugr.es/local/jperez/papers/papers.htm.

123. _ Minimal surfaces whose Gauss map misses four points, Work in progress.

124. - Properly embedded minimal planar domains, Preprint available at http://www.ugr.es/local/jperez/papers/papers.htm.

125. _ Uniqueness of the Riemann minimal examples, Invent. Math. 133 (1998), 107-132. MR.1626477

126. - The geometry of minimal surfaces of finite genus I; curvature estimates and quasiperiodicity, J. Differential Geom. 66 (2004), 1-45. MR2128712 
127. The geometry of minimal surfaces of finite genus II; nonexistence of one limit end examples, Invent. Math. 158 (2004), 323-341. MR2096796

128. _ Liouville-type properties for embedded minimal surfaces, Communications in Analysis and Geometry 14 (2006), no. 4, 703-723. MR2273291

129. , Stable constant mean curvature surfaces, Handbook of Geometrical Analysis, vol. 1, International Press, edited by Lizhen Ji, Peter Li, Richard Schoen and Leon Simon, 2008, pp. 301-380. MR2483369.

130. L Limit leaves of an $H$ lamination are stable, J. Differential Geometry 84 (2010), no. 1, 179-189. MR2629513

131. W. H. Meeks III and H. Rosenberg, The global theory of doubly periodic minimal surfaces, Invent. Math. 97 (1989), 351-379. MR1001845

132. - The maximum principle at infinity for minimal surfaces in flat three-manifolds, Comment. Math. Helvetici 65 (1990), 255-270. MR1057243

133. - The geometry and conformal structure of properly embedded minimal surfaces of finite topology in $\mathbb{R}^{3}$, Invent. Math. 114 (1993), 625-639. MR 1244914

134. 538-578. MR 1241472

135. 858. MR 2182702

136.

137. _ The minimal lamination closure theorem, Duke Math. Journal 133 (2006), no. 3, 467-497. MR 2228460

138. _ Maximum principles at infinity, J. Differential Geometry 79 (2008), no. 1, 141-165. MR 2401421

139. W. H. Meeks III, L. Simon, and S. T. Yau, The existence of embedded minimal surfaces, exotic spheres and positive Ricci curvature, Ann. of Math. (2) 116 (1982), 221-259. MR0678484

140. W. H. Meeks III and M. Weber, Bending the helicoid, Mathematische Annalen 339 (2007), no. 4, 783-798. MR 2341900

141. W. H. Meeks III and B. White, Minimal surfaces bounded by convex curves in parallel planes, Comment. Math. Helvetici 66 (1991), 263-278. MR1107841

142. _ The space of minimal annuli bounded by an extremal pair of planar curves, Communications in Analysis and Geometry 1 (1993), no. 3, 415-437. MR 1266474

143. W. H. Meeks III and M. Wolf, Minimal surfaces with the area growth of two planes; the case of infinite symmetry, Journal of the AMS, 29 (2007) no. 2, 441-465. MR2276776

144. W. H. Meeks III and S. T. Yau, The classical Plateau problem and the topology of threedimensional manifolds, Topology 21 (1982), no. 4, 409-442. MR0670745

145. - The existence of embedded minimal surfaces and the problem of uniqueness, Math. Z. 179 (1982), 151-168. MR0645492

146. J. B. Meusnier, Mémoire sur la courbure des surfaces, Mém. Mathém. Phys. Acad. Sci. Paris, prés. par div. Savans 10 (1785), 477-510, Presented in 1776.

147. V. V. Mīklyukov, Some peculiarities of the behavior of solutions of minimal surface type equations in unbounded domains, Math. Sbornik 116 (1981), no. 1, 72-86, English translation in Math. USSR Sbornik 44(1): 61-73, 1983. MR632489 (83d:35036)

148. V. V. Mīklyukov and A. Weitsman, Carleman's method and solutions to the minimal surface equation, Preprint.

149. P. Mira, Complete minimal Möbius strips in $\mathbb{R}^{n}$ and the Björling problem, J. of Geometry and Physics 56 (2006), 1506-1515. MR2240407(2007d:53012)

150. S. Montiel and A. Ros, Schrödinger operators associated to a holomorphic map, Global Differential Geometry and Global Analysis (Berlin, 1990), Lecture Notes in Mathematics, vol. 1481, Springer-Verlag, 1991, pp. 147-174. MR.1178529

151. C. B. Morrey, The problem of Plateau on a Riemannian manifold, Ann. of Math. (2) 49 (1948), 807-851. MR0027137

152. N. Nadirashvili, Hadamard's and Calabi-Yau's conjectures on negatively curved and minimal surfaces, Invent. Math. 126 (1996), no. 3, 457-465. MR.1419004

153. S. Nayatani, On the Morse index of complete minimal surfaces in Euclidean space, Osaka J. Math. 27 (1990), 441-451. MR 1066637 
154. R. Neel, Brownian motion and the parabolicity of minimal graphs, ArXiv:0810.0669v1 [math.DG].

155. 2440-2472. MR2502522(2010h:58056)

156. H. U. Nissen, Crystal orientation and plate structure in echinoid skeletal units, Science 166 (1969), 1150-1152.

157. J. C. C. Nitsche, A characterization of the catenoid, J. of Math. Mech. 11 (1962), 293-302. MR.0137043

158. _ On new results in the theory of minimal surfaces, Bull. Amer. Math. Soc. 71 (1965), 195-270. MR0173993

159. _ A new uniqueness theorem for minimal surfaces, Arch. Rat. Mech. Anal. 52 (1973), 319-329. MR0341258

160. _ Lectures on minimal surfaces, vol. 1, Cambridge University Press, Cambridge, 1989. MR 1015936

161. R. Osserman, Global properties of minimal surfaces in $E^{3}$ and $E^{n}$, Ann. of Math. (2) 80 (1964), no. 2, 340-364. MR0179701

162. ㄴ The convex hull property of immersed manifolds, J. Differential Geom. 6 (1971), no. 2, 267-270. MR0298595 (45:7647)

163. _ A survey of minimal surfaces, 2nd ed., Dover Publications, New York, 1986. MR.0852409

164. J. Pérez, On singly-periodic minimal surfaces with planar ends, Transactions of the AMS 6 (1997), 2371-2389. MR 1407709

165. A rigidity theorem for periodic minimal surfaces, Comm. in Analysis and Geom. 7 (1999), no. 1, 95-104. MR1674113

166. _ Parabolicity and minimal surfaces, Global theory of minimal surfaces, American Mathematical Society, Providence, RI, for the Clay Mathematics Institute, Cambridge, MA, edited by D. Hoffman, 2005, pp. 163-174. MR2167253

167. _ Uniqueness of the Riemann minimal surfaces, Global theory of minimal surfaces, American Mathematical Society, Providence, RI, for the Clay Mathematics Institute, Cambridge, MA, edited by D. Hoffman, 2005, pp. 597-610. Based on joint work with W.H. Meeks III and A. Ros. MR2167253

168. J. Pérez, Stable embedded minimal surfaces bounded by a straight line, Calculus of Variations and PDE 29 (2007), no. 2, 267-279. MR2307776

169. J. Pérez, M. Rodríguez, and M. Traizet, The classification of doubly periodic minimal tori with parallel ends, Journal of Diff. Geometry 69 (2005), no. 3, 523-577. MR2170278

170. J. Pérez and A. Ros, The space of properly embedded minimal surfaces with finite total curvature., Indiana Univ. Math. J. 45 (1996), no. 1, 177-204. MR.1406689

171. _ Properly embedded minimal surfaces with finite total curvature, The Global Theory of Minimal Surfaces in Flat Spaces, Lecture Notes in Math 1775, Springer-Verlag, 2002, pp. 15-66, G. P. Pirola, editor. MR 1901613

172. J. Pérez and M. Traizet, The classification of singly periodic minimal surfaces with genus zero and Scherk type ends, Transactions of the AMS 359 (2007), no. 3, 965-990. MR2262839

173. I. Peterson, Spiral proof, Science News 168 (2005), no. 25, 393-397.

174. A. V. Pogorelov, On the stability of minimal surfaces, Soviet Math. Dokl. 24 (1981), $274-276$. MR.0630142

175. B. Riemann, Über die Fläche vom kleinsten Inhalt bei gegebener Begrenzung, Abh. Königl, d. Wiss. Göttingen, Mathem. Cl. 13 (1867), 3-52, K. Hattendorf, editor. JFM 01.0218.01.

176. _ Ouevres mathématiques de Riemann, Gauthiers-Villars, Paris, 1898.

177. A. Ros, Compactness of spaces properly embedded minimal surfaces with finite total curvature, Indiana Univ. Math. J. 44 (1995), no. 1, 139-152. MR1336435

178. _ Embedded minimal surfaces: forces, topology and symmetries, Calc. Var. 4 (1996), 469-496. MR:1402733

179. _ , The isoperimetric problem, Global theory of minimal surfaces, American Mathematical Society, Providence, RI, for the Clay Mathematics Institute, Cambridge, MA, edited by D. Hoffman, 2005, pp. 175-209. MR2167260

180. _ One-sided complete stable minimal surfaces, Journal Differential Geometry $\mathbf{7 4}$ (2006), 69-92. MR2260928 
181. H. Rosenberg, Minimal surfaces of finite type, Bull. Soc. Math. France 123 (1995), 351-354. MR 1373739

182. Some recent developments in the theory of minimal surfaces in 3-manifolds, 24th Brazilian Mathematics Colloquium (Instituto de Matematica Pura e Aplicada (IMPA), Rio de Janeiro), IMPA Mathematical Publications, 2003. MR2028922 (2005b:53015)

183. H. Rosenberg, R. Souam, and E. Toubiana, General curvature estimates for stable $H$-surfaces in 3-manifolds and applications, J. Differential Geom. 84 (2010), no. 3, 623-648. MR2669367

184. M. Ross, Schwarz' $P$ and D surfaces are stable, Differential Geom. Appl. 2 (1992), no. 2, 179-195. MR 1245555

185. H. F. Scherk, Bemerkungen über die kleinste Fläche innerhalb gegebener Grenzen, J. R. Angew. Math. 13 (1835), 185-208, ERAM 013.0481cj.

186. M. Schmies, Computational methods for Riemann surfaces and helicoids with handles, Ph.D. thesis, Technical University of Berlin, Berlin, Germany, 2005.

187. A. Schoen, Infinite periodic minimal surfaces without self-intersections, Technical Note D5541, NASA, Cambridge, Mass., May 1970.

188. R. Schoen, Uniqueness, symmetry, and embeddedness of minimal surfaces, J. Differential Geom. 18 (1983), 791-809. MR0730928

189. G. Segal and G. Wilson, Loop groups and equations of $K d V$ type, Publ. Math. de I.H.E.S. 61 (1985), 5-65. MR0783348

190. M. Shiffman, On surfaces of stationary area bounded by two circles, or convex curves, in parallel planes, Ann. of Math. (2) 63 (1956), 77-90. MR0074695

191. B. Solomon, On foliations of $\mathbb{R}^{n+1}$ by minimal hypersurfaces, Comm. Math. Helv. 61 (1986), 67-83. MR0847521

192. M. Soret, Maximum principle at infinity for complete minimal surfaces in flat 3-manifolds, Annals of Global Analysis and Geometry 13 (1995), 101-116. MR 1336206

193. J. Spruck, Two-dimensional minimal graphs over unbounded domains, Journal of the Inst. of Math. Jussieu 1 (2002), no. 4, 631-640. MR 1954438

194. G. Tinaglia, Curvature estimates for minimal surfaces with total boundary curvature less than 4 $\pi$, Proceedings of the American Mathematical Society 137 (2009), 2445-2450. arXiv:0712.1500. MR2495281(2010a:53018)

195. V. G. Tkachev, Disjoint minimal graphs, Annals of Global Analysis and Geometry 35 (2009), no. 2, 139-155. MR2486121 (2010c:53011)

196. M. Traizet, The genus 2 helicoid, Personal Communication.

197. _ An embedded minimal surface with no symmetries, J. Differential Geometry 60 (2002), no. 1, 103-153. MR1924593

198. _ A balancing condition for weak limits of minimal surfaces, Comment. Math. Helvetici 79 (2004), no. 4, 798-825. MR 2099123

199. - On the genus of triply periodic minimal surfaces, J. Differential Geom. 79 (2008), 243-275. MR 2420019

200. _ A minimal surface with one limit end and unbounded curvature, Preprint, 2011.

201. M. Traizet and M. Weber, Hermite polynomials and helicoidal minimal surfaces, Invent. Math. 161 (2005), no. 1, 113-149. MR2178659

202. M. Tsuji, Potential theory in modern function theory, 2nd ed., Chelsea Publishing Company, New York, NY, 1975. MR0414898

203. J. Tysk, Eigenvalue estimates with applications to minimal surfaces, Pacific J. of Math. 128 (1987), 361-366. MR0888524

204. M. Weber and M. Wolf, Minimal surfaces of least total curvature and moduli spaces of plane polygonal arcs, Geom. Funct. Anal. 8 (1998), 1129-1170. MR1664793

205. 156 (2002), 713-795. MR1954234

206. A. Weitsman, Growth of solutions to the minimal surface equation over domains in a half plane, Communications in Analysis and Geometry 13 (2005), 1077-1087. MR 2216153

207. _ On the growth of minimal graphs, Indiana Univ. Math Journal 54 (2005), no. 2, 617-625. MR 2136824

208. F. Xavier, The Gauss map of a complete non-flat minimal surface cannot omit 7 points of the sphere, Ann. of Math. (2) 113 (1981), 211-214. MR0604048

209. - Convex hulls of complete minimal surfaces, Math. Ann. 269 (1984), 179-182. MR 0759107 
210. S.T. Yau, Problem section, Seminar on Differential Geometry, Annals of Math. Studies, vol. 102, 1982, pp. 669-706. MR0645762

211. L Review of geometry and analysis, Mathematics: frontiers and prospectives, Amer. Math. Soc., Providence, RI, 2000, pp. 353-401. MR1754787

Department of Mathematics, University of Massachusetts, Amherst, Massachusetts 01003

E-mail address: profmeeks@gmail.com

Department of Geometry and Topology, University of Granada, Granada, Spain

E-mail address: jperez@ugr.es 